\title{
Crossed Products by Endomorphisms, Vector Bundles and Group Duality
}

\author{
Ezio Vasselli \\ Dipartimento di Matematica \\ University of Rome "Tor Vergata" \\ vasselli@mat.uniroma2.it \\ http://mat.uniroma2.it/ vasselli/
}

September 4, 2018

\begin{abstract}
We construct the crossed product $\mathcal{A} \rtimes^{\mathcal{E}} \widehat{\rho}$ of a $C^{*}$-algebra $\mathcal{A}$ with centre $\mathcal{Z}$ by an endomorphism $\rho$, which is special in a weaker sense w.r.t. the notion introduced by Doplicher and Roberts. The notation $\widehat{\rho}$ denotes the tensor $C^{*}$-category of powers of $\rho$. We assign to $\rho$ a geometrical invariant, representing a cohomological obstruction to be special in the usual sense, and determining rank and first Chern class of the vector bundle $\mathcal{E}$ whose module of sections (contained in $\mathcal{A} \rtimes^{\mathcal{E}} \widehat{\rho}$ ) induces $\rho$ on $\mathcal{A}$. We prove that $\mathcal{A}$ is the fixed point $C^{*}$-algebra w.r.t. a $G$-action on $\mathcal{A} \rtimes^{\mathcal{E}} \widehat{\rho}$, and characterize $\widehat{\rho}$ as the category of tensor powers of a suitable $G$-Hilbert $\mathcal{Z}$-bimodule (a so-called 'noncommutative pullback' of $\mathcal{E}$ ). $G$ is interpreted as the generally noncompact space of sections of a group bundle.
\end{abstract}

AMS Subj. Class.: 46L05, 46L08, 22D35

\section{Introduction and Preliminaries.}

Let $\mathcal{A}$ be a unital $C^{*}$-algebra with centre $\mathcal{Z}$. For each pair of endomorphisms $\rho, \sigma$ we consider the intertwiners spaces $(\rho, \sigma):=\{t \in \mathcal{A}: \sigma(a) t=t \rho(a), a \in \mathcal{A}\}$. Every $(\rho, \sigma)$ has an obvious structure of Banach $\mathcal{Z}$-bimodule $z, t \mapsto z t$, with $z \in \mathcal{Z}, t \in(\rho, \sigma)$; in particular, when $\rho$ is the identity automorphism $\iota$ we get a Hilbert $\mathcal{Z}$-bimodule endowed with the natural $\mathcal{Z}$-valued scalar product $t, t^{\prime} \mapsto$ $t^{*} t^{\prime}$, where $t, t^{\prime} \in(\iota, \sigma)$ (see [14] for an exposition about Hilbert bimodules in 
$C^{*}$-algebras). We say that an endomorphism $\rho$ is inner if $(\iota, \rho)$ is non zero and finitely generated as a right Hilbert $\mathcal{Z}$-module, i.e. if there is a finite set $\left\{\psi_{l}\right\} \subset(\iota, \rho)$ such that $\rho(1)=\sum_{l} \psi_{l} \psi_{l}^{*}$. Our terminology is justified by the fact that if $\rho$ is inner then for $a \in \mathcal{A}$

$$
\rho(a)=\sum_{l} \rho(a) \psi_{l} \psi_{l}^{*}=\sum_{l} \psi_{l} a \psi_{l}^{*}
$$

so that $\rho$ is induced by every set of generators of $(\iota, \rho)$, playing a role analogue to the unitaries for inner automorphisms. Viceversa, every finitely generated Hilbert $\mathcal{Z}$-bimodule contained in $\mathcal{A}$ in the sense above induces, via (1.1), an inner endomorphism of $\mathcal{A}$. The notion of inner endomorphism has been considered and used in [15] in the case in which $\mathcal{A}$ has trivial centre; the terminology adopted in that context for $(\iota, \rho)$ is Hilbert space in a $C^{*}$-algebra, as the scalar product is $\mathbb{C}$-valued.

In analogy with the case of a $\mathbb{Z}$-action on a $C^{*}$-algebra one can define the crossed product by an endomorphism implementing a $\mathbb{N}$-action, as in 32, [1], 22. In this context the endomorphism is induced by an isometry (a one dimensional Hilbert space in our terminology), in analogy with the usual crossed product by an automorphism. After this work more general constructions have been done, implementing the endomorphism by a Hilbert space, as for example in 38, but previously in [19]. In this last paper the crossed product of a $C^{*}$ algebra by an endomorphism is related to duality theory for compact groups in the following sense: the set of endomorphisms of $\mathcal{A}$, with arrows the intertwiners spaces $(\rho, \sigma)$, is a strict tensor $C^{*}$-category (in the sense of [18]) called $\operatorname{end} \mathcal{A}$, when endowed with the tensor structure

$$
\left\{\begin{aligned}
\rho, \sigma & \mapsto \rho \sigma:=\rho \circ \sigma \\
t, t^{\prime} & \mapsto t \times t^{\prime}:=t \rho\left(t^{\prime}\right)=\rho^{\prime}\left(t^{\prime}\right) t \in\left(\rho \sigma, \rho^{\prime} \sigma^{\prime}\right)
\end{aligned}\right.
$$

where $t \in\left(\rho, \rho^{\prime}\right), t^{\prime} \in\left(\sigma, \sigma^{\prime}\right)$. Recalling classical duality theories one could ask which are the conditions such that some full subcategory of end $\mathcal{A}$ is isomorphic to the dual of a compact group. Basic step for the answer is given in the case of a single endomorphism by the following definitions and theorem:

Definition 1.1 (Permutation symmetry). A unital endomorphism $\rho$ of $\mathcal{A}$ has permutation symmetry if there is a unitary representation $p \mapsto \varepsilon(p)$ of the group $\mathbb{P}_{\infty}$ of finite permutations of $\mathbb{N}$ in $\mathcal{A}$ such that:

$$
\begin{gathered}
\varepsilon(\mathbb{S} p)=\rho \circ \varepsilon(p) \\
\varepsilon:=\varepsilon(1,1) \in\left(\rho^{2}, \rho^{2}\right) \\
\varepsilon(s, 1) t=\rho(t) \varepsilon(r, 1), t \in\left(\rho^{r}, \rho^{s}\right)
\end{gathered}
$$


where $(r, s) \in \mathbb{P}_{r+s}$ permutes the first $r$ terms with the remaining $s$ and $\mathbb{S}$ is the shift $(\mathbb{S} p)(1):=1,(\mathbb{S} p)(n):=1+p(n-1), p \in \mathbb{P}_{\infty}$. Above properties imply that

$$
\varepsilon(p) \in\left(\rho^{n}, \rho^{n}\right), n \in \mathbb{N}, p \in \mathbb{P}_{n}
$$

we say that $\rho$ has weak permutation symmetry if just (1.2), 1.5) hold.

Definition 1.2 (Special Conjugate Property). Let $\rho$ be an endomorphism of $\mathcal{A}$ with permutation symmetry. $\rho$ has dimension $d>1$ and satisfies the special conjugate property if there exists an isometry $R \in\left(\iota, \rho^{d}\right)$ such that

- $R^{*} \rho(R)=(-1)^{d-1} d^{-1} 1$

- $R R^{*}=P_{\varepsilon, d}:=\frac{1}{d !} \sum_{p \in \mathbb{P}_{d}} \operatorname{sign}(p) \varepsilon(p)$

where $\mathbb{P}_{d}$ is the permutations group of $d$ objects.

Previous definitions model at algebraic level respectively the symmetry of tensor product and the existence of conjugate for a representation of a compact group. An endomorphism satisfying Def 1.2 is called special (according to terminology of 18 ). Let us now denote by $\widehat{\rho}$ the full strict tensor $C^{*}$-subcategory of end $\mathcal{A}$ generated by the powers of $\rho$. In this setting it can be proved the following

Theorem $1.3([19])$. Let $\mathcal{A}$ be a unital $C^{*}$-algebra with trivial centre, $\rho$ a special endomorphism of $\mathcal{A}$. Then there is a $C^{*}$-algebra $\mathcal{A} \rtimes \widehat{\rho}$, a Hilbert space $H$ in $\mathcal{A} \rtimes \widehat{\rho}$ of dimension $d$, and a faithful strongly continuous action $\alpha$ of a closed subgroup $G \subset \mathbb{S U}(d)$ on $\mathcal{A} \rtimes \widehat{\rho}$ satisfying the following properties:

- $\alpha$ coincides with the natural $G$-action on $H$;

- there is a unital inclusion $\mathcal{A} \hookrightarrow \mathcal{A} \rtimes \widehat{\rho}$ with $\mathcal{A}=(\mathcal{A} \rtimes \widehat{\rho})^{\alpha}$;

- $\left.\sigma_{H}\right|_{\mathcal{A}}=\rho$, where $\sigma_{H}$ is the inner endomorphism induced by $H$;

- $\mathcal{A}, H$ generate $\mathcal{A} \rtimes \widehat{\rho}$ as a $C^{*}$-algebra, and $\mathcal{A}^{\prime} \cap(\mathcal{A} \rtimes \widehat{\rho})=\mathbb{C} 1$.

By above crossed product construction it is very easy to deduce a characterization of $\widehat{\rho}$ as the category of tensor powers of the defining representation of $G$. Previous theorem is an important step toward the Doplicher-Roberts duality for compact groups and is motivated by questions arising in the context of algebraic quantum field theory (see [18 and related references). It has also been applied to nontrivial centre case in [6], maintaining (in essence) properties Def 1.1 Def 1.2 the result (in the case of a single endomorphism) is a crossed product where the endomorphism becomes induced by a free module of the type $\mathbb{C}^{d} \otimes \mathcal{Z}$, and a group $G \subseteq \mathbb{S U}(d)$ is recovered as in previous theorem.

As pointed up before in general the natural objects providing the notion of inner endomorphism are (not free) Hilbert $C^{*}$-bimodules over the centre of the 
given $C^{*}$-algebra, i.e., by Serre-Swan Theorem, modules of continuous sections of vector bundles. In the present paper, that is a continuation of [0], we generalize previous quoted crossed product constructions by considering nontrivial centre, and by relaxing permutation symmetry and special conjugate property, which do not hold in the cases we consider (as canonical endomorphisms of Pimsner algebras, that we exhibit as crossed products). We obtain an algebraic duality theory, characterizing categories of $C^{*}$-algebra endomorphisms as categories of $G$-Hilbert bimodules. The group $G$ we reconstruct is interpreted as the (noncompact) space of continuous sections of a Lie group bundle, in general not trivial and with not constant fibre (thus a more general object w.r.t. [19, [6]), and belonging to a class including continuous loop groups. Our results allow to describe some basic structures of the tensor product in the category of $G$-Hilbert bimodules (as symmetry, conjugates) that have different properties w.r.t. the case of $G$-Hilbert spaces. A basic tool and example in our work is the Pimsner algebra associated to the module of continuous sections of a vector bundle, that plays a role analogue to the Cuntz algebra in [19. It is proved in [40] (but it was previously and indipendentely recognized by J.E.Roberts [37]) that this $C^{*}$-algebra has the structure of a continuous field of Cuntz algebras. Next sections are organized as follows.

In Section 2 we construct the crossed product (Prop 2.3) of a $C^{*}$-algebra $\mathcal{A}$ by an endomorphism $\rho$, in such a way that $\rho$ becomes induced by the module of continuous sections of a vector bundle over the spectrum $\widehat{\mathcal{Z}}^{\rho}$ of the abelian $C^{*}$ algebra $\mathcal{Z}^{\rho}:=\left\{f \in \mathcal{A} \cap \mathcal{A}^{\prime}: \rho(f a)=f \rho(a), a \in \mathcal{A}\right\}$. Our construction, made in the context of $C(X)$-actions (see [36, [20, 21] and related references), includes as particular cases (i.e. trivial vector bundles) the crossed products by Stacey [38, Cuntz 11] and Paschke 32.

In Section 3 we introduce the $C^{*}$-algebra associated to a closed group of unitaries on a vector bundle. We prove an analogue of Tannaka-Krein duality for such groups (Prop 3.6). An interesting fact is that non isomorphic groups may have isomorphic categories of tensor powers of the defining representation (Cor 3.11). The reason is non unicity of embedding into the category of vector bundles.

In Section 4 we introduce and describe a class of Hilbert bimodules corresponding to noncommutative pullbacks of vector bundles, in the sense of Def. 4.4 They naturally appear in the context of our crossed products (Prop 5.11), but not only there $(35,, 29$, , 23] $)$. We characterize such noncommutative pullbacks in terms of properties of the associated semitensor $C^{*}$-category of tensor powers (Prop 4.5). Furthermore we study group actions over noncommutative pullbacks and associated Pimsner algebras.

In Section 5 we go through the same line of [19] and generalize the notion of dual action of a Lie group on a $C^{*}$-algebra $\mathcal{A}$. If $G$ is a closed group of unitaries 
on a vector bundle $\mathcal{E} \rightarrow X$, a dual action is a functor $\mu: \widehat{G} \rightarrow$ end $\mathcal{A}$, where $\widehat{G}$ is the category of tensor powers of the defining representation $i d$ of $G$ over $\mathcal{E}$. In this setting we construct a crossed product (Thm. [5.9), that comes equipped with the module of continuous sections of $\mathcal{E}$ inducing on $\mathcal{A}$ the endomorphism $\mu(i d)$. The crossed product is acted upon by $G$ in such a way that the fixed point algebra is isomorphic to $\mathcal{A}$.

In Section 6 we study $C^{*}$-algebra endomorphisms which are special according to generalized (less restrictive) notions of permutation symmetry (Def 6.3) and special conjugate property (Def [6.9). In particular we allow existence of intertwiners that do not satisfy (1.4); otherwise we will talk about symmetry intertwiners (Def 6.1). We assign to special endomorphisms $\rho$ a geometrical invariant $c(\rho):=c_{0}(\rho) \oplus c_{1}(\rho)$ (Def[6.12), belonging to the sheaf cohomology group $H^{0}\left(\widehat{\mathcal{Z}}^{\rho}, \mathbb{Z}\right) \oplus H^{2}\left(\widehat{\mathcal{Z}}^{\rho}, \mathbb{Z}\right)$. First summand in $H^{0}\left(\widehat{\mathcal{Z}}^{\rho}, \mathbb{Z}\right)$ corresponds to a generalized dimension of permutation symmetry, while second summand in $H^{2}\left(\widehat{\mathcal{Z}}^{\rho}, \mathbb{Z}\right)$ denotes a cohomological obstruction to get the special conjugate property in the sense of Def 1.2 Special endomorphisms in the sense of [19] thus correspond to elements of the type $d \oplus 0, d \in \mathbb{N}$.

Section 7 contains our main results. We prove that if $\rho$ is a special endomorphism in the sense of Def6.12 then for every vector bundle $\mathcal{E} \rightarrow \widehat{\mathcal{Z}}^{\rho}$ with rank $c_{0}(\rho)$ and first Chern class $c_{1}(\rho)$ a natural dual action of the group $\mathbf{S U E}$ of special unitaries of $\mathcal{E}$ is induced on $(\mathcal{A}, \rho)$ (Thm. 7.1). Finally, we prove the analogue of Thm 1.3 for some (up to coniugacy) unique subgroup $G \subseteq \mathbf{S U E}$ (Thm [7.8). We also give duality results: we prove that $\hat{\rho}$ is isomorphic to the strict tensor $C^{*}$-category of tensor powers of a unitary representation of $G$ over a noncommutative pullback of $\mathcal{E}$. Furthermore the subcategory of symmetry intertwiners in $\widehat{\rho}$ is isomorphic to the strict tensor $C^{*}$-category of tensor powers of the defining representation of $G$ over $\mathcal{E}$ (Cor 7.10).

Last section 8 is devoted to a brief exposition about further developements, involving duality for abstract tensor categories, $K$-theory, braiding.

\section{Crossed Products by Endomorphisms and Vec- tor Bundles.}

Let $\rho$ be an endomorphism of a $C^{*}$-algebra $\mathcal{A}$. Then $\rho$ induces a natural structure of $C(X)$-algebra on $\mathcal{A}$; in fact if $M(\mathcal{A})$ is the multipliers algebra of $\mathcal{A}$ with centre $Z M(\mathcal{A})$ we define

$$
\mathcal{Z}^{\rho}:=\{f \in Z M(\mathcal{A}): \rho(f a)=f \rho(a), a \in \mathcal{A}\} .
$$

Being $\mathcal{Z}^{\rho}$ a unital subalgebra of $Z M(\mathcal{A})$ it is clear that $\mathcal{A}$ is a $\mathcal{Z}^{\rho}$-algebra. Furthermore by definition $\rho$ is equivariant w.r.t. $\mathcal{Z}^{\rho}$-action. Also note that 
$\mathcal{Z}^{\rho}$ is an invariant of the sector $\operatorname{sect}(\rho):=\left\{u \rho(\cdot) u^{*} \in \operatorname{end} \mathcal{A}, u \in U M(\mathcal{A})\right\}$. Previous elementary remark suggests to consider $C(X)$-algebras as a natural framework to study endomorphisms with related crossed product constructions.

Let now $X$ be a locally compact Hausdorff space, $\mathcal{A}$ a $C_{0}(X)$-algebra, $\rho$ a $C_{0}(X)$-endomorphism on $\mathcal{A}$. Given a vector bundle $\mathcal{E} \rightarrow X$ with compact support (i.e. $\mathcal{E}$ is trivial in a neighborhood of the point at infinity: in other terms, $\mathcal{E}$ defines a vector bundle over the one point compactification $X^{+}$), we want to construct a crossed product of $\mathcal{A}$ by $\rho$ in such a way that the endomorphism becomes induced by the module $\widehat{\mathcal{E}}$ of continuous sections of $\mathcal{E}$. Before to proceed recall that every $C_{0}(X)$-algebra $\mathcal{A}$ can be faithfully represented over a suitable Hilbert $C_{0}(X)^{\prime \prime}$-module $\mathcal{M}$ by a $C_{0}(X)$-monomorphism (see [27]). Also note that $C\left(X^{+}\right)$is contained in the centre of the $C^{*}$-algebra $\mathcal{L}(\mathcal{M})$ of right $C_{0}(X)^{\prime \prime}$-module endomorphisms of $\mathcal{M}$.

Definition 2.1. A covariant representation of $\mathcal{A}, \rho$ with rank $\mathcal{E}$ is a pair $\left(\pi, \mathcal{E}_{\pi}\right)$, where

- $\pi: M(\mathcal{A}) \rightarrow \mathcal{L}\left(\mathcal{M}_{\pi}\right)$ is a unital $C_{0}(X)$-representation of the multipliers algebra $M(\mathcal{A})$ over a Hilbert $C_{0}(X)^{\prime \prime}$-module $\mathcal{M}_{\pi}$;

- $\mathcal{E}_{\pi}$ is a Hilbert $C\left(X^{+}\right)$-bimodule in $\mathcal{L}\left(\mathcal{M}_{\pi}\right)$ isomorphic to $\widehat{\mathcal{E}}$ such that the relation $\pi \circ \rho=\sigma_{\pi} \circ \pi$ holds, where $\sigma_{\pi}$ is the inner endomorphism induced by $\mathcal{E}_{\pi}$ on $\mathcal{L}\left(\mathcal{M}_{\pi}\right)$ via [1.1]).

We are going to construct our crossed product in such a way that a universality condition is satisfied w.r.t. covariant representations, whose existence we are going to prove. At this purpose, let us denote by $\mathcal{O}_{\mathcal{E}}$ the Pimsner algebra of $\widehat{\mathcal{E}}$ (see [40] or Sec 3 for basic properties of $\mathcal{O}_{\mathcal{E}}$ ); recall that $\mathcal{O}_{\mathcal{E}}$ may be regarded as the $C^{*}$-algebra generated by the $C\left(X^{+}\right)$-bimodules $\left(\mathcal{E}^{r}, \mathcal{E}^{s}\right)$ of vector bundle morphisms between the tensor powers $\mathcal{E}^{r}, \mathcal{E}^{s}, r, s \in \mathbb{N}$, according to the procedure exposed in [14], 18], or [28] (in particular, there is a natural isomorphism $\widehat{\mathcal{E}} \simeq(\iota, \mathcal{E})$, where $\left.\iota:=\mathcal{E}^{0}:=X \times \mathbb{C}\right)$. It is clear that every covariant representation $\left(\pi, \mathcal{E}_{\pi}\right)$ induces a faithful representation $\mathcal{O}_{\mathcal{E}} \rightarrow \mathcal{L}\left(\mathcal{M}_{\pi}\right)$. We furthermore introduce the inductive limit $\mathcal{A}_{\infty}:=\mathcal{A} \stackrel{\rho}{\rightarrow} \mathcal{A} \stackrel{\rho}{\rightarrow} \cdots$.

Lemma 2.2. Let $\mathcal{E} \rightarrow X$ be a vector bundle with compact support. There exist covariant representations $\left(\pi, \mathcal{E}_{\pi}\right)$ of $\mathcal{A}, \rho$ with rank $\mathcal{E}$ if and only if $\mathcal{A}_{\infty} \neq\{0\}$.

Proof. We proceed as in [38] and related references. Let $\mathcal{A}_{\infty} \neq\{0\}$. We consider the crossed product $\mathcal{A} \rtimes_{\rho} \mathbb{N}$ of $\mathcal{A}$ by $\rho$ with canonical maps $\mathcal{A} \stackrel{\rho_{0}}{\rightarrow}$ $\mathcal{A}_{\infty} \stackrel{i_{\infty}}{\hookrightarrow} \mathcal{A} \rtimes_{\rho} \mathbb{N}$ and define $i_{\mathcal{A}}:=i_{\infty} \circ \rho_{0}$. Recall that $\mathcal{A} \rtimes_{\rho} \mathbb{N}$ is obtained by the crossed product $\mathcal{A}_{\infty} \rtimes_{\rho_{\infty}} \mathbb{Z}$ of $\mathcal{A}_{\infty}$ by the automorphism $\rho_{\infty}$ induced by $\rho$, with canonical injection $j_{\infty}: \mathcal{A}_{\infty} \hookrightarrow \mathcal{A}_{\infty} \rtimes_{\rho_{\infty}} \mathbb{Z}$, as the corner $\mathcal{A} \rtimes_{\rho} \mathbb{N}:=p_{\infty} \cdot \mathcal{A}_{\infty} \rtimes_{\rho_{\infty}}$ $\mathbb{Z} \cdot p_{\infty}$, where $p_{\infty}:=j_{\infty} \circ \rho_{0}(1)$ (here 1 denotes the identity of $M(\mathcal{A})$, note that by surjectivity $\rho_{0}$ naturally extends on the multipliers algebra). Let us now prove that $\mathcal{A} \rtimes_{\rho} \mathbb{N}$ is a $C_{0}(X)$-algebra. As $\mathcal{A}_{\infty} \neq\{0\}$ we find that $\rho_{0}(a)$ is non 
zero for some $a \in \mathcal{A}$. Now for every $f \in C_{0}(X)$ we find $\rho^{k}(f a)=f \rho^{k}(a) \neq 0$ thus, regarding at this identity in $\mathcal{A}_{\infty}$, we find $\rho_{\infty} \circ \rho_{0}(f a)=\rho_{0}(f) \cdot \rho_{\infty} \circ$ $\rho_{0}(a)$. Let now $v \in \mathcal{A} \rtimes_{\rho} \mathbb{N}$ be the canonical isometry such that $i_{\mathcal{A}} \circ \rho(a)=$ $v i_{\mathcal{A}}(a) v^{*}$, with $p:=v v^{*}$. The relation $\rho(f a)=f \rho(a)$ implies $i_{\mathcal{A}}(f) v i_{\mathcal{A}}(a) v^{*}=$ $v i_{\mathcal{A}}(f) i_{\mathcal{A}}(a) v^{*}$, so that (being $i_{\mathcal{A}}$ non degenerate) $i_{\mathcal{A}}(f)$ commutes with $i_{\mathcal{A}}(a)$, $v$. Thus $\mathcal{A} \rtimes_{\rho} \mathbb{N}$ is a $C_{0}(X)$-algebra. By [27] we know that there exist a faithful $C_{0}(X)$-module representation $\nu$ of $\mathcal{A} \rtimes_{\rho} \mathbb{N}$ over a Hilbert $C_{0}(X)^{\prime \prime}$ module $\mathcal{M}$. Furthermore we consider a faithful $C\left(X^{+}\right)$-module representation $\nu^{\prime}$ of $\mathcal{O}_{\mathcal{E}}$ over a Hilbert $C_{0}(X)^{\prime \prime}$-module $\mathcal{M}^{\prime}$. Now (following [27], Def. 1.6) we consider the (ungraded) tensor product $\mathcal{M} \otimes_{C_{0}(X)^{\prime \prime}} \mathcal{M}^{\prime}$ and claim that the pair $\left(\left(\nu \circ i_{\mathcal{A}}\right) \otimes 1, \nu(v) \otimes \nu^{\prime}(\widehat{\mathcal{E}})\right)$ is a covariant representation of $\mathcal{A}$ over $\mathcal{M} \otimes_{C_{0}(X)^{\prime \prime}}$ $\mathcal{M}^{\prime}$. In fact it is obvious that $\nu(v) \otimes \nu^{\prime}(\widehat{\mathcal{E}})$ is isomorphic to $\widehat{\mathcal{E}}$ as a Hilbert $C\left(X^{+}\right)$-bimodule, and has support ${ }^{1}$

$$
\sum_{l}\left(\nu(v) \otimes \psi_{l}\right) \cdot\left(\nu(v)^{*} \otimes \psi_{l}^{*}\right)=\left(\nu(v) \cdot \nu\left(v^{*}\right)\right) \otimes 1=\nu(p) \otimes 1,
$$

where $\left\{\psi_{l}\right\}$ is a finite set of generators of $\nu^{\prime}(\widehat{\mathcal{E}})$. If $a \in \mathcal{A}, \psi \in \nu^{\prime}(\widehat{\mathcal{E}})$ then

$$
\begin{aligned}
\left(\nu \circ i_{\mathcal{A}}(\rho(a)) \otimes 1\right) \cdot(\nu(v) \otimes \psi) & =\nu\left(i_{\mathcal{A}}(\rho(a)) \cdot v\right) \otimes \psi= \\
& =\nu\left(v \cdot i_{\mathcal{A}}(a) \cdot v^{*} \cdot v\right) \otimes \psi= \\
& =\nu\left(v \cdot i_{\mathcal{A}}(a)\right) \otimes \psi= \\
& =(\nu(v) \otimes \psi) \cdot\left(\nu \circ i_{\mathcal{A}}(a) \otimes 1\right)
\end{aligned}
$$

and the first implication is proved. Viceversa if $\mathcal{A}_{\infty}=\{0\}$ then for every $a \in \mathcal{A}$ there exists $k \in \mathbb{N}$ such that $\rho^{k}(a)=0$. Thus if $\left(\pi, \mathcal{E}_{\pi}\right)$ is a covariant representation then $0=\pi\left(\rho^{k}(a)\right)=\sum_{|L|=k} \psi_{L} \pi(a) \psi_{L}^{*}$, and $\pi(a)=0$ because $\pi \circ \rho^{k}$ has left inverse ${ }^{2}$ the map $a \mapsto \frac{1}{d^{k}} \sum_{L} \psi_{L}^{*} \pi(a) \psi_{L}$, where $d$ is the rank of $\mathcal{E}$. Thus we find a contradiction, and the lemma is proved.

Proposition 2.3. Let $\mathcal{A}$ be a $C_{0}(X)$-algebra and $\rho$ a $C_{0}(X)$-endomorphism of $\mathcal{A}$ with $\mathcal{A}_{\infty} \neq\{0\}$. Then for every vector bundle $\mathcal{E} \rightarrow X$ with compact support there exists up to isomorphism a unique $C_{0}(X)$-algebra $\mathcal{A} \rtimes_{\rho}^{\mathcal{E}} \mathbb{N}$ such that

- there is a non degenerate $C_{0}(X)$-morphism $i: \mathcal{A} \rightarrow \mathcal{A} \rtimes_{\rho}^{\mathcal{E}} \mathbb{N}$;

\footnotetext{
${ }^{1}$ The support of a Hilbert bimodule $\mathcal{M}$ in a $C^{*}$-algebra is defined by the formula $p_{\mathcal{M}}:=$ $\sum \psi_{l} \psi_{l}^{*}$, where $\left\{\psi_{l}\right\}$ is a finite set of generators of $\mathcal{M}$. It is trivial to check that $p_{\mathcal{M}}$ does not depend on the choice of the generators.

${ }^{2}$ We recall that a left inverse of an endomorphism $\rho$ is a positive linear map $\phi: \mathcal{A} \rightarrow \mathcal{A}$ such that $\phi(1)=1$ and $\phi(a \rho(b))=\phi(a) b$ for every $a, b \in \mathcal{A}$.
} 
- the module $\widehat{\mathcal{E}}$ of continuous sections of $\mathcal{E}$ is contained in $M\left(\mathcal{A} \rtimes_{\rho}^{\mathcal{E}} \mathbb{N}\right)$ as a finitely generated Hilbert $C\left(X^{+}\right)$-bimodule, and $i \circ \rho=\sigma_{\mathcal{E}} \circ i$, where $\sigma_{\mathcal{E}}$ is the inner endomorphism induced by $\widehat{\mathcal{E}}$;

- $\mathcal{A} \rtimes_{\rho}^{\mathcal{E}} \mathbb{N}$ is generated as a $C^{*}$-algebra by $i(\mathcal{A}), \widehat{\mathcal{E}}$;

- for every covariant representation $\left(\pi, \mathcal{E}_{\pi}\right)$ with rank $\mathcal{E}$ there is a unique non degenerate $C_{0}(X)$-representation $\Pi: \mathcal{A} \rtimes_{\rho}^{\mathcal{E}} \mathbb{N} \rightarrow \mathcal{L}\left(\mathcal{M}_{\pi}\right)$ such that $\Pi \circ i=\pi$ and $\Pi(\widehat{\mathcal{E}})=\mathcal{E}_{\pi}$.

Proof. We consider the ${ }^{*}$-algebra $\mathcal{B}$ generated by $\mathcal{A}, \widehat{\mathcal{E}}$ with relations

$$
\left\{\begin{array}{l}
\psi^{*} \psi^{\prime}=\left\langle\psi, \psi^{\prime}\right\rangle, \quad f \psi=\psi f \\
(a f) \cdot \psi=a \cdot(f \psi) \\
\psi \cdot a=\rho(a) \cdot \psi
\end{array}\right.
$$

where $a \in \mathcal{A}, \psi, \psi^{\prime} \in \widehat{\mathcal{E}}, f \in C_{0}(X)$ and $\langle\cdot, \cdot\rangle$ is the $C\left(X^{+}\right)$-valued scalar product on $\widehat{\mathcal{E}}$. We consider a set $\Gamma$ of covariant representations such that every covariant representation of $\mathcal{A}$ is equivalent to a unique direct sum of elements of $\Gamma$. Now, every covariant representation induces a representation of $\mathcal{B}$, and the closure of $\mathcal{B}$ w.r.t. the $C^{*}$-norm $\|\cdot\|:=\sup _{\gamma \in \Gamma}\|\gamma(\cdot)\|$ is by construction the required crossed product.

Next proposition states the compatibility of our crossed product with those by Stacey, Paschke, Cuntz, by choosing trivial vector bundles.

Proposition 2.4. Let $\mathcal{A}$ be a $C_{0}(X)$-algebra, $\rho$ a $C_{0}(X)$-endomorphism of $\mathcal{A}$. With $[d]:=X \times \mathbb{C}^{d}$ denoting the trivial rank $d$ vector bundle over $X, \mathcal{A} \rtimes_{\rho}^{[d]} \mathbb{N}$ is canonically isomorphic to the crossed product $\mathcal{A} \rtimes_{\rho}^{d} \mathbb{N}$ (in the sense of Stacey, [38]).

Proof. The space of continuous sections of $[d]$ is isomorphic to the free rank $d$ Hilbert $C\left(X^{+}\right)$-module. Thus we can pick $d$ orthonormal generators $\psi_{1}, \ldots, \psi_{d}$, that appear in $\mathcal{A} \rtimes_{\rho}^{[d]} \mathbb{N}$ as isometries such that $\psi_{i}^{*} \psi_{j}=\delta_{i j} 1$ and inducing the inner endomorphism extending $\rho$. Of course both $\mathcal{A} \rtimes_{\rho}^{[d]} \mathbb{N}$ and $\mathcal{A} \rtimes_{\rho}^{d} \mathbb{N}$ are generated by elements of the type $i(a) \psi_{L} \psi_{M}^{*}$; thus we just have to verify that $\mathcal{A} \rtimes_{\rho}^{d} \mathbb{N}$ satisfies universal property w.r.t. covariant representations $\left(\pi,[d]_{\pi}\right)$ (note that $\mathcal{A} \rtimes_{\rho}^{d} \mathbb{N}$ is a $C_{0}(X)$-algebra, so it makes sense to consider $C_{0}(X)$-module representations of $\left.\mathcal{A} \rtimes_{\rho}^{d} \mathbb{N}\right)$. Let now $\mathcal{H}$ be the Hilbert $C_{0}(X)^{\prime \prime}$-module carrying the covariant representation $\left(\pi,[d]_{\pi}\right)$; we denote by $\psi_{i}^{\pi}$ the orthonormal isometries in $\mathcal{L}(\mathcal{H})$ generating $[d]_{\pi}$. Note that by definition if $z \in C_{0}(X)$ then $\pi(z) v=v z$ for every $v \in \mathcal{H}$. We have to prove that there exists a $C_{0}(X)$-module representation $\Pi$ of $\mathcal{A} \rtimes_{\rho}^{d} \mathbb{N}$ as in Prop 2.3 We consider a faithful state $\lambda$ on $C_{0}(X)^{\prime \prime}$, and the Hilbert space $\mathcal{H}_{\lambda}$ obtained by introducing on $\mathcal{H}$ the $\mathbb{C}$-valued scalar product $(v, w)_{\lambda}:=\lambda(v, w), v, w \in \mathcal{H}$. Thus there is a unital monomorphism $i_{\lambda}: \mathcal{L}(\mathcal{H}) \hookrightarrow \mathcal{L}\left(\mathcal{H}_{\lambda}\right)$, and $\left(i_{\lambda} \circ \pi,\left\{i_{\lambda}\left(\psi_{i}^{\pi}\right)\right\}_{i=1}^{d}\right)$ is a covariant representation in 
the sense of [38]. By construction of $\mathcal{A} \rtimes_{\rho}^{d} \mathbb{N}$ there exists a representation $\Pi_{\lambda}$ of $\mathcal{A} \rtimes_{\rho}^{d} \mathbb{N}$ such that $\pi_{\lambda}=\Pi_{\lambda} \circ i$ and $\Pi_{\lambda}\left(\psi_{i}\right)=i_{\lambda}\left(\psi_{i}^{\pi}\right)$. Now, since $i_{\lambda}$ is injective and $\Pi_{\lambda}\left(\mathcal{A} \rtimes_{\rho}^{d} \mathbb{N}\right) \subseteq i_{\lambda}(\mathcal{L}(\mathcal{H}))$ we can define the $C_{0}(X)$-module representation $\Pi:=\left.i_{\lambda}^{-1}\right|_{i_{\lambda}(\mathcal{L}(\mathcal{H}))} \circ \Pi_{\lambda}$. As $\Pi$ satisfies by construction the required properties the proposition is proved.

Remark 2.1. Let $\mathcal{A}$ be a continous field of $C^{*}$-algebras over $X$. If the morphism $\mathcal{A} \rightarrow \mathcal{A} \rtimes_{\rho}^{\mathcal{E}} \mathbb{N}$ is injective then the crossed product $\mathcal{A} \rtimes_{\rho}^{\mathcal{E}} \mathbb{N}$ is a continuous field over $X$ itself. In fact $\mathcal{A} \rtimes_{\rho}^{\mathcal{E}} \mathbb{N}$ is a $C_{0}(X)$-algebra, and the norm function is continuous for elements of the dense set generated by $i(\mathcal{A})$ and $\widehat{\mathcal{E}}$.

Remark 2.2. Let $\rho$ be an automorphism of a $C^{*}$-algebra $\mathcal{A}, \mathcal{L} \rightarrow \widehat{\mathcal{Z}}^{\rho}$ a line bundle. Then the inner endomorphism $\sigma_{\mathcal{L}}$ is a locally unitary automorphism of $\mathcal{A} \rtimes{ }_{\rho}^{\mathcal{L}} \mathbb{N}$ in the sense of 36.

Example 2.1. Let $\mathcal{A}$ be a continuous trace $C^{*}$-algebra with spectrum $X$. Then for every $C(X)$-endomorphism $\rho$ of $\mathcal{A}$ there exists a unique (up to isomorphism) finitely generated Hilbert $C(X)$-bimodule $\widehat{\mathcal{E}}(\rho)$ in the multipliers algebra $M(\mathcal{A})$, inducing $\rho$ on $\mathcal{A}$ in the usual way (1.1) (see 23). $\widehat{\mathcal{E}}(\rho)$ is isomorphic to the module of continuous sections of a vector bundle $\mathcal{E}(\rho) \rightarrow X$, and the $C^{*}$-subalgebra of $M(\mathcal{A})$ generated by $\mathcal{A}, \widehat{\mathcal{E}}(\rho)$ is isomorphic to the crossed product $\mathcal{A} \rtimes_{\rho}^{\mathcal{E}(\rho)} \mathbb{N}$, since obviously satisfies universal property w.r.t. covariant representations.

Example 2.2. Let $\mathcal{E} \rightarrow X$ be a vector bundle. Let furthermore $\mathcal{O}_{\mathcal{E}}^{0} \simeq$ $\lim _{\rightarrow r}\left(\mathcal{E}^{r}, \mathcal{E}^{r}\right)$ be the fixed point $C^{*}$-algebra of $\mathcal{O}_{\mathcal{E}}$ w.r.t. the canonical circle action $z \mapsto\{\psi \mapsto z \psi, \psi \in \widehat{\mathcal{E}}\}, z \in \mathbb{T}$. If $p \in(\mathcal{E}, \mathcal{E})$ is a projection we define the shift endomorphism $\widehat{p}(t):=t \mapsto p \otimes t, t \in\left(\mathcal{E}^{r}, \mathcal{E}^{r}\right)$. Note that $\widehat{p}=p \cdot \widehat{1}$, so that $\widehat{p}$ is a subobject of the canonical endomorphism $\hat{1}$ in the category end $\mathcal{O}_{\mathcal{E}}^{0}$. Let in particular be $p$ a rank 1 projection and $\rho:=\widehat{p}$. We prove that $\mathcal{O}_{\mathcal{E}} \simeq \mathcal{O}_{\mathcal{E}}^{0} \rtimes_{\rho}^{\mathcal{L}} \mathbb{N}$, where $\mathcal{L}:=p \mathcal{E}$ is the line subbundle of $\mathcal{E}$ spanned by $p$. In fact $\mathcal{O}_{\mathcal{E}}$ is generated by $\mathcal{O}_{\mathcal{E}}^{0}$ and $\widehat{\mathcal{L}}$, because $\widehat{\mathcal{E}}=(\mathcal{E}, \mathcal{E}) \cdot \widehat{\mathcal{L}}$. Furthermore $\rho$ becomes inner in $\mathcal{O}_{\mathcal{E}}$ : in fact the identity $(p \otimes t) \cdot\left(\psi \otimes^{r} 1\right)=\psi \otimes t, t \in\left(\mathcal{E}^{r}, \mathcal{E}^{r}\right), \psi \in p \widehat{\mathcal{E}} \simeq \widehat{\mathcal{L}}$ is translated as $\rho(t) \psi=\psi t$ in $\mathcal{O}_{\mathcal{E}}$. Finally we have to prove that $\mathcal{O}_{\mathcal{E}}$ satisfies universal property for covariant representations. If $\left(\pi: \mathcal{O}_{\mathcal{E}}^{0} \rightarrow \mathcal{L}(\mathcal{H}), \mathcal{L}_{\pi}\right)$ is a covariant representation then we define $\mathcal{E}_{\pi}:=\pi(\mathcal{E}, \mathcal{E}) \cdot \mathcal{L}_{\pi}$. Now note that since $p$ has rank 1 then $(\rho, \rho) \simeq(\mathcal{L}, \mathcal{L}) \simeq p \cdot(\mathcal{E}, \mathcal{E}) \cdot p=C(X) \cdot p$. If $t, t^{\prime} \in(\mathcal{E}, \mathcal{E})$, $\psi, \psi^{\prime} \in \mathcal{L}_{\pi}$ we find $\psi^{*} \pi\left(t^{*} t^{\prime}\right) \psi^{\prime}=\psi^{*} \pi(p) \pi\left(t^{*} t^{\prime}\right) \pi(p) \psi^{\prime}=f \psi^{*} \psi^{\prime}$ for some $f \in C(X)$. Thus $\psi^{*} \pi\left(t^{*} t^{\prime}\right) \psi^{\prime} \in C(X)$ and $\mathcal{E}_{\pi}$ is a Hilbert $C(X)$-bimodule in $\mathcal{L}(\mathcal{H})$, that is obviously isomorphic to $\widehat{\mathcal{E}}$. The natural $C(X)$-morphism $\Pi: \mathcal{O}_{\mathcal{E}} \rightarrow C^{*}\left\{\mathcal{E}_{\pi}\right\} \subset \mathcal{L}(\mathcal{H})$ is then the desired representation extending $\pi$. 


\section{The $C^{*}$-algebra of a $G$-Vector Bundle.}

Let $\mathcal{E}$ be a rank $d$ vector bundle ${ }^{3}$ over a compact Hausdorff space $X, \mathbf{U} \mathcal{E}$ the group of unitary maps of $\mathcal{E}$. Being the $C^{*}$-algebra $(\mathcal{E}, \mathcal{E})$ of endomorphisms of $\mathcal{E}$ a locally trivial continuous field of matrix algebras, $\mathbf{U E}$ can be naturally regarded as the space of continuous sections of a locally trivial group bundle $\mathcal{U E} \rightarrow X$, that we call the unitary bundle of $\mathcal{E}$ (see for instance [12] or 24]), having fibre $\mathbb{U}(d)$ and structure group $\mathbb{U}(d)$ acting on itself by adjoint action. If $\left\{u_{i j}\right\} \in H^{1}(X, \mathbb{U}(d))$ is a set of transition functions associated to $\mathcal{E}$ for an open trivializing cover $\left\{X_{i}\right\}$, then $\mathcal{U E}$ can be constructed by pasting together the spaces $X_{i} \times \mathbb{U}(d)$ via the maps $f_{i j}(x, u):=\left(x, u_{i j}(x) \cdot u \cdot u_{i j}^{*}(x)\right)$, with $(x, u) \in\left(X_{i} \cap X_{j}\right) \times \mathbb{U}(d)$. Thus elements of $\mathbf{U E}$ can be locally described by families of continuous maps $g_{i}: X_{i} \rightarrow \mathbb{U}(d)$ satisfying the cocycle relations $u_{i j} g_{j}=g_{i} u_{i j}$. If $\mathcal{E}$ is trivial then $\mathbf{U} \mathcal{E}=C(X, \mathbb{U}(d))$, i.e. the group of continuous maps from $X$ into $\mathbb{U}(d)$.

We furthermore consider the determinant function det : $\mathbf{U E} \rightarrow C(X, \mathbb{T})$ defined by $\operatorname{det}(g)(x):=\operatorname{det}\left(g_{x}\right)$, and introduce the group of special unitaries SUE $:=\operatorname{det}^{-1}\{1\}$. It is well known that $\mathbf{S U E}$ corresponds to a locally trivial bundle $\mathcal{S U E} \rightarrow X$ with fibre $\mathbb{S U}(d)$.

We now briefly recall basic properties of the Pimsner $C^{*}$-algebra $\mathcal{O}_{\mathcal{E}}$ of the module $\widehat{\mathcal{E}}$ of continuous sections of $\mathcal{E}$ (see [40 for details). A first important point is that $\mathcal{O}_{\mathcal{E}}$ is the $C^{*}$-algebra of a locally trivial continuous field of Cuntz algebras over $X$. Furthermore $\mathcal{O}_{\mathcal{E}}$ is endowed with a canonical shift endomorphism $\sigma$ in the following way: if we denote by $\left(\mathcal{E}^{r}, \mathcal{E}^{s}\right)$ the $C(X)$-bimodule of vector bundle morphisms between tensor powers $\mathcal{E}^{r}, \mathcal{E}^{s}$, then $\sigma(t):=1 \otimes t$, $t \in\left(\mathcal{E}^{r}, \mathcal{E}^{s}\right) \subset \mathcal{O}_{\mathcal{E}}$, where 1 is the identity on $\mathcal{E} . \sigma$ is inner, with $(\iota, \sigma) \simeq \widehat{\mathcal{E}}$, and amenability $\left(\mathcal{E}^{r}, \mathcal{E}^{s}\right)=\left(\sigma^{r}, \sigma^{s}\right)$ holds (in the sense of [28]). Note that $\sigma$ carries permutation symmetry, but in general does not have a well defined dimension in the usual sense (in fact dimension of canonical endomorphism corresponds to the not necessarily constant rank of $\mathcal{E}$ ), and does not satisfy special conjugate property Def 1.2 unless vanishing of the first Chern class, as we will see in the sequel. Also note that $\sigma$ is a $C(X)$-module map (i.e. is the identity on $\left.C(X) \subset \mathcal{O}_{\mathcal{E}}\right)$. We recall the definition of canonical $\mathbf{U} \mathcal{E}$-action on $\mathcal{O}_{\mathcal{E}}$ :

$$
\widehat{g}(t):=g^{\otimes^{s}} \cdot t \cdot g^{* \otimes^{r}}
$$

where $g \in \mathbf{U} \mathcal{E}, t \in\left(\mathcal{E}^{r}, \mathcal{E}^{s}\right)$.

Let now be $G$ a closed subgroup of $\mathbf{U} \mathcal{E}$; we consider the ${ }^{*}$-subalgebra ${ }^{0} \mathcal{O}_{G}$ of $\mathcal{O}_{\mathcal{E}}$ generated by the Banach $C(X)$-bimodules

$$
\left(\mathcal{E}^{r}, \mathcal{E}^{s}\right)_{G}:=\left\{y \in\left(\mathcal{E}^{r}, \mathcal{E}^{s}\right): \widehat{g}(y)=y, g \in G\right\}
$$

\footnotetext{
${ }^{3}$ As customary we will often assume for simplicity that the rank of a vector bundle is constant. Recall that by local triviality rank has to be constant over connected components of the base space.
} 
and denote by $\mathcal{O}_{G}$ the closure of ${ }^{0} \mathcal{O}_{G}$ in $\mathcal{O}_{\mathcal{E}}$. The action (3.1) by automorphisms on $\mathcal{O}_{\mathcal{E}}$ is compatible with $\mathbb{Z}$-grading and inductive limit structure (see [16], [18]); thus we find that $\mathcal{O}_{G}$ is the fixed point $C^{*}$-algebra w.r.t. $G$-action on $\mathcal{O}_{\mathcal{E}} \cdot \mathcal{O}_{G}$ naturally inherits by $\mathcal{O}_{\mathcal{E}}$ the structure of continuous field of $C^{*}$ algebras, with fibre $\left(\mathcal{O}_{G}\right)_{x} \subseteq \mathcal{O}_{d}$. Furthermore if $g$ is any element of $\mathbf{U} \mathcal{E}$ then for $t \in\left(\mathcal{E}^{r}, \mathcal{E}^{s}\right)$ we find $\widehat{g} \circ \sigma(t)=\widehat{g}(1 \otimes t)=1 \otimes \widehat{g}(t)$, so that $\widehat{g} \circ \sigma=\sigma \circ \widehat{g}$; thus for every $G \subseteq \mathbf{U} \mathcal{E}$ is well defined the restriction $\sigma_{G}$ on $\mathcal{O}_{G}$ of the canonical endomorphism. For intertwiners as usual we use the notation

$$
\left(\sigma_{G}^{r}, \sigma_{G}^{s}\right):=\left\{y \in \mathcal{O}_{G}: y \sigma_{G}^{r}\left(y^{\prime}\right)=\sigma_{G}^{s}\left(y^{\prime}\right) y, y^{\prime} \in \mathcal{O}_{G}\right\}
$$

We now discuss structural properties of $\mathcal{O}_{G}$ and canonical endomorphism $\sigma_{G}$. Let us denote by $\lambda \mathcal{E}$ the exterior tensor product with order the rank of $\mathcal{E}$. $\lambda \mathcal{E}$ is a line bundle, and $\lambda\left(\mathcal{E} \oplus \mathcal{E}^{\prime}\right)=\lambda \mathcal{E} \otimes \lambda \mathcal{E}^{\prime}$ (see for example 24] about above identity) so that, by the usual identification of $H^{2}(X, \mathbb{Z})$ with the group of isomorphism classes of line bundles over $X$ (endowed with the tensor product as group operation) we obtain a homomorphism $c_{1}$ from $K(X)$ onto $H^{2}(X, \mathbb{Z})$, i.e. the first Chern class. Let us give a local description of $\lambda \mathcal{E}$; consider a trivialization $\pi_{i}:\left.\mathcal{E}\right|_{X_{i}} \mapsto X_{i} \times \mathbb{C}^{d}$ such that $\mathcal{E}$ admits a family $\left\{u_{i j}:=\pi_{i} \pi_{j}^{-1}\right\}$ of transition functions taking values in the unitary group $\mathbb{U}(d)$. Pick a partition of unity $\left\{\lambda_{i}\right\}$ subordinate to the open cover $\left\{X_{i}\right\}$ and consider, for each $i$, the continuous sections $R_{i}:=\lambda_{i}\left(\pi_{i}^{\otimes^{d}}\right)^{-1}(R)$ of $\mathcal{E}^{d}$, where

$$
R:=\sum_{p \in \mathbb{P}_{d}} \operatorname{sign}(p) e_{p(1)} \otimes \ldots \otimes e_{p(d)} ;
$$

here $\mathbb{P}_{d}$ is the permutations group and $\left\{e_{h}\right\}$ is the standard orthonormal basis of $\mathbb{C}^{d}$. The $R_{i}$ 's, as elements of the Hilbert $C(X)$-module of sections of $\mathcal{E}^{d}$ satisfy the relations

$$
\begin{gathered}
\left\langle R_{i}, R_{j}\right\rangle=\lambda_{i} \lambda_{j}\left\langle R, u_{i j}^{\otimes^{d}} R\right\rangle=\lambda_{i} \lambda_{j} \operatorname{det}\left(u_{i j}\right) \in C(X) ; \\
\lambda_{i} R_{j}=\lambda_{j} \operatorname{det}\left(u_{i j}\right) R_{i}
\end{gathered}
$$

(here we used the fact that the local charts $\pi_{i}$ preserve the scalar product) and generate $(\iota, \lambda \mathcal{E})$. SUE -action on $(\iota, \lambda \mathcal{E}) \subset\left(\iota, \mathcal{E}^{d}\right)$ is trivial, since $\widehat{g}\left(R_{i}\right)=$ $\operatorname{det}(g) R_{i}=R_{i}$ for $g \in \mathbf{S U} \mathcal{E}$. Now, the support in $\mathcal{O}_{\mathcal{E}}$ of $(\iota, \lambda \mathcal{E})$ is given by the projection

$$
P:=\sum_{i} R_{i} R_{i}^{*}
$$

so that for $g \in \mathbf{U} \mathcal{E}$ and by using (3.5) we find $\widehat{g}(P)=\operatorname{det}(g) P \operatorname{det}\left(g^{*}\right)=P$. Previous considerations imply that $(\iota, \lambda \mathcal{E}) \subset \mathcal{O}_{\text {SUE }}$ and $P \in \mathcal{O}_{\mathbf{U} \mathcal{E}}$. 
An element of $\left(\mathcal{E}^{2}, \mathcal{E}^{2}\right)$ that will play a special role in the sequel is the symmetry $\theta \in\left(\mathcal{E}^{2}, \mathcal{E}^{2}\right)$ such that

$$
\theta\left(\psi \otimes \psi^{\prime}\right):=\psi^{\prime} \otimes \psi
$$

where $\psi, \psi^{\prime} \in(\iota, \mathcal{E})$. It is clear that $\theta=\theta^{*}=\theta^{-1}$. As an element of $\mathcal{O}_{\mathcal{E}}$ the symmetry $\theta$ can be expressed in the following way:

$$
\theta=\sum_{l, m} \psi_{m} \psi_{l} \psi_{m}^{*} \psi_{l}^{*}
$$

where as usual $\left\{\psi_{l}\right\}$ is a set of generators of $(\iota, \mathcal{E})$. Since right $C(X)$-action coincides with the left one on $(\iota, \mathcal{E})$ we obtain that $\theta$ is actually well defined (i.e. does not depend on the choice of generators), bypassing the problems mentioned in [5]. Thus, for $g \in \mathbf{U} \mathcal{E}$ we find

$$
\widehat{g}(\theta)=\sum_{l, m}\left(g \psi_{m}\right)\left(g \psi_{l}\right)\left(g \psi_{m}\right)^{*}\left(g \psi_{l}\right)^{*}=\theta
$$

and $\theta \in \mathcal{O}_{\mathbf{U} \mathcal{E}}$. By using canonical endomorphism and symmetry we get, with the same methods in [16], a representation

$$
p \mapsto \theta(p) \in\left(\mathcal{E}^{p}, \mathcal{E}^{p}\right): \quad \theta(p) \cdot \otimes_{i} \psi_{i}:=\otimes_{i} \psi_{p(i)}, \quad \psi_{i} \in(\iota, \mathcal{E})
$$

of $\mathbb{P}_{\infty}$ in $\mathcal{O}_{\mathcal{E}}$ such that $\theta \circ \mathbb{S}(p)=\sigma \circ \theta(p)$. Note that each $\theta(p)$ is a word in $\theta$, $\sigma(\theta), \ldots, \sigma^{s}(\theta)$ for some $s \in \mathbb{N}$. Since canonical endomorphism commutes with $\mathbf{U} \mathcal{E}$-action $\theta(p)$ belongs to $\mathcal{O}_{\mathbf{U E}}$ for each $p$. In particular we will make use of the unitary operators $\theta(r, s)$ on $\mathcal{E}^{r+s}$ permuting first $r$ factors of the tensor product $\mathcal{E}^{r} \otimes \mathcal{E}^{s}$ with remaining $s$. Elementary properties of these operators, that are the same for vector bundles as well for Hilbert spaces, are given in [16]. We will make use of the obvious identity

$$
\theta(s, 1) \cdot(t \otimes 1)=(1 \otimes t) \cdot \theta(r, 1)
$$

$t \in\left(\mathcal{E}^{r}, \mathcal{E}^{s}\right)$, that regarded in $\mathcal{O}_{\mathcal{E}}$ becomes

$$
\theta(s, 1) \cdot t=\sigma(t) \cdot \theta(r, 1)
$$

Note that in particular $\theta \psi=\sigma(\psi)$ for $\psi \in(\iota, \mathcal{E})$. By using (3.9) and considerations above we conclude that $\sigma$ (and more generally the restrictions $\sigma_{G}$ ) has permutation symmetry in the sense of Def 1.2 Now locally we have $R^{*} \sigma(R)=(-1)^{d-1} d^{-1}$ (see [19]) and this fact implies the following global identity, that will be used in next sessions:

$$
\sum_{i} R_{i}^{*} \sigma\left(R_{i}\right)=(-1)^{d-1} d^{-1} 1
$$


In the same way the local identity $R R^{*}=\frac{1}{d !} \sum_{p \in \mathbb{P}_{d}} \operatorname{sign}(p) \theta(p)$ implies

$$
P=\sum_{i} R_{i} R_{i}^{*}=\frac{1}{d !} \sum_{p \in \mathbb{P}_{d}} \operatorname{sign}(p) \theta(p)
$$

so that $P$ belongs to the $\sigma$-stable algebra generated by $\theta$.

\section{Proposition 3.1.}

- $\sigma(t)=\lim _{r \rightarrow \infty} \theta(r+k, 1) \cdot t \cdot \theta(r, 1)^{*}$, for every $t \in \mathcal{O}_{\mathcal{E}}^{k}$;

- if $t \in \mathcal{O}_{\mathcal{E}}$ and $[t, \theta(p)]=0$ for $p \in \mathbb{P}_{\infty}$ then $\sigma(t)=t$ and $t \in C(X)$;

- if $G$ is any subgroup of $\mathbf{U E}$ then $\mathcal{O}_{G}^{\prime} \cap \mathcal{O}_{\mathcal{E}}=C(X)$;

- amenability holds, i.e. $\left(\mathcal{E}^{r}, \mathcal{E}^{s}\right)_{G}=\left(\sigma_{G}^{r}, \sigma_{G}^{s}\right)$ for $r, s \in \mathbb{N}$.

Proof.

- It follows immediately by (3.9) and passing to the inductive limit.

- If $t$ commutes with $\theta(p)$ for every $p \in \mathbb{P}_{\infty}$ then $t_{x} \theta_{x}(p)=\theta_{x}(p) t_{x}$ for each fibre $t_{x} \in \mathcal{O}_{d}$ and the corresponding permutation operator $\theta_{x}(p)$ at varying of $x$ in $X$. Thus by [16, Lemma 3.2 we find $t_{x} \in \mathbb{C}$.

- Since $\theta \in \mathcal{O}_{\mathbf{U E}} \subset \mathcal{O}_{G}$ and $\mathcal{O}_{G}$ is $\sigma_{G}$-stable we find that $t \in \mathcal{O}_{G}^{\prime} \cap \mathcal{O}_{\mathcal{E}}$ commutes with $\theta(p)$ for every $p \in \mathbb{P}_{\infty}$, so that $t \in C(X)$.

- If $t \in\left(\sigma_{G}^{r}, \sigma_{G}^{s}\right)$ then $t_{L M}:=\psi_{L}^{*} t \psi_{M} \in \mathcal{O}_{G}^{\prime} \cap \mathcal{O}_{\mathcal{E}}=C(X)$ for $\psi_{M} \in\left(\iota, \mathcal{E}^{r}\right)$, $\psi_{M} \in\left(\iota, \mathcal{E}^{s}\right)$. Thus $t=\sum_{L M} t_{L M} \psi_{L} \psi_{M}^{*} \in\left(\mathcal{E}^{r}, \mathcal{E}^{s}\right)$ and is by definition $G$-invariant. Viceversa if $t \in\left(\mathcal{E}^{r}, \mathcal{E}^{s}\right)_{G}$ then $t \in\left(\mathcal{E}^{r}, \mathcal{E}^{s}\right)=\left(\sigma^{r}, \sigma^{s}\right)$; since by hypothesis $t \in \mathcal{O}_{G}$, in particular $t$ belongs to $\left(\sigma_{G}^{r}, \sigma_{G}^{s}\right)$.

Previously exposed properties (in particular amenability) allow us to give the following

Definition 3.2. Let $\mathcal{E} \rightarrow X$ be a vector bundle, $G \subseteq \mathbf{U E}$ a closed group. We denote by $\widehat{G}$ the symmetric strict tensor $C^{*}$-category with objects the tensor powers $\mathcal{E}^{r}, r \in \mathbb{N}$, and arrows the invariant $C(X)$-bimodules $\left(\mathcal{E}^{r}, \mathcal{E}^{s}\right)_{G} . \widehat{G}$ is said the dual of $G$.

Let us introduce for every $x \in X$ the closed group

$$
K_{x}:=\left\{g_{x}: g \in G\right\} \subseteq \mathbb{U}(d) .
$$

Now, we know that $\mathcal{O}_{G}$ defines a continuous field of $C^{*}$-algebras over $X$; anyway in general $\left(\mathcal{O}_{G}\right)_{x} \neq \mathcal{O}_{K_{x}}$, where $\mathcal{O}_{K_{x}} \subseteq \mathcal{O}_{d}$ is the fixed point algebra w.r.t. natural $K_{x}$-action over $\mathcal{O}_{d}$ (as defined in [16]): 
Lemma 3.3. Let $\mathcal{E} \rightarrow X$ be a rank $d$ vector bundle, $G \subseteq \mathbf{U E}$ a closed group. Then for every $x \in X$ there is a closed group $G_{x} \subseteq \mathbb{U}(d)$ with $\left(\mathcal{O}_{G}\right)_{x} \subseteq \mathcal{O}_{G_{x}}$. $G_{x}$ is defined as the group of automorphisms of $\mathcal{O}_{d}$ leaving $\left(\mathcal{O}_{G}\right)_{x}$ pointwise fixed, and there is an inclusion $K_{x} \subseteq G_{x}$. If $G$ is the group of sections of locally trivial bundle, or is contained in $\mathbf{S U \mathcal { E }}$, then $\left(\mathcal{O}_{G}\right)_{x}=\mathcal{O}_{G_{x}}$.

Proof. Since $\mathcal{O}_{G}$ is $\sigma$-stable, so is $\left(\mathcal{O}_{G}\right)_{x} \subseteq \mathcal{O}_{d}$. We denote by $\sigma_{x}$ the canonical endomorphism on $\left(\mathcal{O}_{G}\right)_{x}$. Note that $\left(\mathcal{O}_{G}\right)_{x}$ is generated by elements of the intertwiners spaces $\left(\sigma_{x}^{r}, \sigma_{x}^{s}\right)$, and that because of symmetry of $\sigma_{x}$ the equality $\left(\mathcal{O}_{G}\right)_{x}^{\prime} \cap \mathcal{O}_{d}=\mathbb{C}$ holds. Let now $\alpha \in \operatorname{aut}_{\left(\mathcal{O}_{G}\right)_{x}} \mathcal{O}_{d}$; then if $\left\{s_{i}\right\}_{i=1}^{d}$ is a set of isometries generating $\mathcal{O}_{d}$ we find $s_{i}^{*} \alpha\left(s_{j}\right) \in\left(\mathcal{O}_{G}\right)_{x}{ }^{\prime} \cap \mathcal{O}_{d}=\mathbb{C}$. Thus $G_{x}:=$ aut $_{\left(\mathcal{O}_{G}\right)_{x}} \mathcal{O}_{d}$ can be identified as a closed subgroup of $\mathbb{U}(d)$, acting on $\mathcal{O}_{d}$ by the canonical action (3.1), and $\left(\mathcal{O}_{G}\right)_{x} \subseteq \mathcal{O}_{G_{x}}$. If $g \in G$ then $\widehat{g}(y)=y$ for every $y \in\left(\mathcal{E}^{r}, \mathcal{E}^{s}\right)_{G}$, i.e. $\widehat{g}_{x}\left(y_{x}\right)=y_{x}$ for every $x \in X$. Thus $\widehat{g}_{x}$ is an automorphism of $\mathcal{O}_{d}$ leaving $\left(\mathcal{O}_{G}\right)_{x}$ pointwise fixed, and $g_{x} \in G_{x}$, i.e. $K_{x} \subseteq G_{x}$. Finally, if $G \subseteq$ SUE then $\sigma_{x}$ is special for every $x \in X$ in the sense of 19, and $\mathcal{O}_{d}=\left(\mathcal{O}_{G}\right)_{x} \rtimes \widehat{\sigma}_{x}$. Thus $\left(\mathcal{O}_{G}\right)_{x}$ is the fixed point algebra w.r.t. $G_{x}$-action on $\mathcal{O}_{d}$, and $\left(\mathcal{O}_{G}\right)_{x}=\mathcal{O}_{G_{x}}$. If the bundle spanned by $G$ is locally trivial with fibre $G_{0}$ and $t \in \mathcal{O}_{G_{0}}$ we consider a cutoff function $\lambda \in C(X)^{+}$vanishing out of a trivializing set for $\mathcal{E}$, with $\lambda(x)=1$. The vector field $\bar{t}:=\int_{G_{0}} \widehat{g}(\lambda \cdot t) d g$ belongs to $\mathcal{O}_{G}$, and $\bar{t}_{x}=t$. Thus $\mathcal{O}_{G_{0}} \subseteq\left(\mathcal{O}_{G}\right)_{x}$, and $\left(\mathcal{O}_{G}\right)_{x}=\mathcal{O}_{G_{x}}$.

Note that the order of Cuntz algebra $\mathcal{O}_{d}$ (i.e. the rank of $\mathcal{E}$ in $x$ ) can be recovered by analyzing permutation symmetry of the canonical endomorphism in $\left(\mathcal{O}_{G}\right)_{x}$ (in particular dimension, by using methods in [16]). So that $d$ is completely determined by properties of $\mathcal{O}_{G}$.

Example 3.1. Let $X:=[0,1], \mathcal{E}:=X \times \mathbb{C}^{d}, G:=\left\{g \in C(X, \mathbb{U}(d)): g\left(x_{0}\right)=\mathbf{1}\right\}$, with $0<x_{0}<1$. Then $K_{x}=\mathbb{U}(d)$ for every $x \neq x_{0}$, while $K_{x_{0}}=\{\mathbf{1}\}$. It is clear that $\left(\mathcal{E}^{r}, \mathcal{E}^{s}\right)=C(X) \otimes \mathbb{M}_{d^{r}, d^{s}}$, where $\mathbb{M}_{d^{r}, d^{s}}$ is the Banach space of $d^{r} \times d^{s}$-matrices. Suppose now that $t \in\left(\mathcal{E}^{r}, \mathcal{E}^{s}\right)$ is $G$-invariant. Then for every neighbourhood $U$ of $x_{0}$ we find that $\left.t\right|_{X-U}$ is a norm-continuous map taking values into the space of $d^{r} \times d^{s}$-matrices invariant w.r.t. action by $\mathbb{U}(d)$ (defined as in (3.1)). By [16], $\left.t\right|_{X-U}$ is zero for $r \neq s$, and takes values into the permutation group $C^{*}$-algebra $C^{*} \mathbb{P}_{r}$ for $r=s$. By continuity $t\left(x_{0}\right)=0$ if $r \neq s$, while for $r=s$ we find that $t\left(x_{0}\right) \in C^{*} \mathbb{P}_{r}$. Thus $\left(\mathcal{E}^{r}, \mathcal{E}^{r}\right)_{G}=C(X) \otimes C^{*} \mathbb{P}_{r}\left(\right.$ with $\left.C^{*} \mathbb{P}_{r}=\left(\mathbb{C}^{d^{r}}, \mathbb{C}^{d^{r}}\right)_{\mathbb{U}(d)}\right)$, and $\left(\mathcal{O}_{G}\right)_{x}=\mathcal{O}_{\mathbb{U}(d)}$ $\forall x \in X$. So that $G_{x}=\mathbb{U}(d)=\operatorname{aut}_{\mathcal{O}_{\mathbb{U}(d)}} \mathcal{O}_{d}$ for every $x \in X$, having applied [16], Cor. 3.3.

The group $G_{x}$ defined in previous lemma is said to be the fibre of $G$ over $x \in X$. Note that if $g \in G$ then $g_{x} \in G_{x}$ for every $x \in X$.

Definition 3.4. Let $\mathcal{E} \rightarrow X$ be a rank d vector bundle, $G \subseteq \mathbf{U E}$ a closed group. The spectral bundle $\mathcal{G} \rightarrow X$ spanned by $G$ is the set $\mathcal{G}:=\bigsqcup_{x} G_{x} \subset \bigsqcup_{x} \mathbb{U}(d)$, endowed with the topology induced as a subset of the unitary bundle $\mathcal{U E}$. 
Note that $\mathcal{G}$ is a compact Hausdorff space. We denote by the notation $S(X, \mathcal{G})$ the closed group of elements of $\mathbf{U} \mathcal{E}$ spanning $\mathcal{G}$ as continuous sections. By definition it is clear that $G \subseteq S(X, \mathcal{G})$. Note that there is a simple criteria to determine whether some $g \in \mathbf{U} \mathcal{E}$ is an element of $S(X, \mathcal{G})$ : it suffices in fact to verify that $g_{x} \in G_{x}$ for every $x \in X$.

A subgroup $G$ of $\mathbf{U} \mathcal{E}$ does not define locally trivial spectral bundles in general (an example: $\left.\mathcal{E}:=[0,3] \times \mathbb{C}^{d}, G:=\{g \in C([0,3], \mathbb{U}(d)): g(t)=\mathbf{1}, t \in[1,2]\}\right)$. In the sequel we will explicitly specify when $G$ defines a (locally) trivial bundle $\mathcal{G} \rightarrow X$ by calling it (locally) trivial itself. When $X$ is connected local triviality implies that the fibre $G_{0} \subseteq \mathbb{U}(d)$ of $\mathcal{G}$ is constant: thus we have local charts $\left.\mathcal{G}\right|_{X_{i}} \simeq X_{i} \times G_{0}$, and elements of $S(X, \mathcal{G})$ can be locally described by continuous maps $g_{i}: X_{i} \rightarrow G_{0}$ satisfying the cocycle relations $u_{i j} g_{j}=g_{i} u_{i j}$, where $u_{i j}: X_{i} \cap X_{j} \rightarrow \mathbb{U}(d)$ are transition functions of $\mathcal{E}$. If $\mathcal{G}$ is trivial then $S(X, \mathcal{G})=C\left(X, G_{0}\right)$ is a continuous loop group.

Example 3.2. Let $\mathcal{E} \rightarrow X$ be a vector bundle. Then the spectral bundle spanned by $\mathbf{U} \mathcal{E}$ is the unitary bundle $\mathcal{U E}$. In the same way, the spectral bundle spanned by $\mathbf{S U E}$ is the special unitary bundle $\mathcal{S U E}$. These bundles are trivial if $\mathcal{E}$ is the tensor product of a trivial bundle by a line bundle.

Lemma 3.5. Let $G \subset \mathbf{U E}$ be a closed group. Then $\widehat{G}=\widehat{G^{\prime}}$ for every closed group $G^{\prime} \subseteq \mathbf{U E}$ spanning the same spectral bundle of $G$. In particular, $\widehat{G}=$ $S(X, \mathcal{G})$ and $\mathcal{O}_{G}=\mathcal{O}_{S(X, \mathcal{G})}$.

Proof. If $t \in\left(\mathcal{E}^{r}, \mathcal{E}^{s}\right)_{G}$ then $t_{x} \in\left(\mathcal{O}_{G}\right)_{x}$ for every $x \in X$, thus $t_{x}$ is $G_{x}$ invariant by previous lemma. This fact implies that if $g \in S(X, \mathcal{G})$ then $\widehat{g}(t)=$ $t$, since $(\widehat{g}(t))_{x}=\widehat{g}_{x}\left(t_{x}\right)=t_{x}$ being $g_{x} \in G_{x}$. Now if $G^{\prime}$ spans the same spectral bundle of $G$ we have $g^{\prime}{ }_{x} \in G_{x}$ for every $g^{\prime} \in G^{\prime}$, thus $\widehat{g^{\prime}}(t)=t$. So that we proved that $\left(\mathcal{E}^{r}, \mathcal{E}^{s}\right)_{G} \subseteq\left(\mathcal{E}^{r}, \mathcal{E}^{s}\right)_{G^{\prime}}$. By exchanging the role of $G, G^{\prime}$ we obtain $\left(\mathcal{E}^{r}, \mathcal{E}^{s}\right)_{G}=\left(\mathcal{E}^{r}, \mathcal{E}^{s}\right)_{G^{\prime}}$.

Example 3.3. Refering to Example 3.1 we obtain that the spectral bundle of $G$ is $X \times \mathbb{U}(d)$, and $\widehat{G}=C(X, \mathbb{U}(d))$. In the same way, if $G^{\prime}:=G \cap C(X, \mathbb{S U}(d))$, we find that the spectral bundle of $G^{\prime}$ is $X \times \mathbb{S U}(d)$, and $\widehat{G}^{\prime}=C(X, \mathbb{S U}(d))$.

Example 3.4. We consider a closed group $G \subseteq \mathbb{U}(d)$ acting on the trivial vector bundle $\mathcal{E}:=X \times \mathbb{C}^{d}$ by the obvious action $\xi \mapsto g \cdot \xi, g \in G, \xi \in \mathcal{E}_{x} \simeq \mathbb{C}^{d}$. The spectral bundle of $G$ is $\mathcal{G}=X \times G$, with $S(X, \mathcal{G})=C(X, G)$, i.e. the group of continuous maps from $X$ into $G$. It is trivially verified that

$$
\left(\mathcal{E}^{r}, \mathcal{E}^{s}\right)_{G}=\left(\mathcal{E}^{r}, \mathcal{E}^{s}\right)_{C(X, G)}=C(X) \otimes\left(H_{G}^{r}, H_{G}^{s}\right)
$$

where $H_{G} \subset \mathbb{C}^{d}$ is the Hilbert space of $G$-invariant vectors. Thus $\widehat{G}=C(X, G)$.

We can now prove a Tannaka duality in the setting of vector bundles; in analogy with [16], Cor. 3.3, the following result allows the reconstruction of 
(the spectral bundle associated to) $G$ by the triple $\left(\mathcal{O}_{G}, \sigma_{G}, \mathcal{E}\right)$ thus, because of amenability, by the dual $\widehat{G}$. We denote by $\operatorname{aut}_{\mathcal{O}_{G}} \mathcal{O}_{\mathcal{E}}$ the group of automorphisms of $\mathcal{O}_{\mathcal{E}}$ coinciding with the identity on $\mathcal{O}_{G}$.

Proposition 3.6. For every closed group $G \subseteq \mathbf{U E}$ there is a natural isomorphism $S(X, \mathcal{G}) \simeq \operatorname{aut}_{\mathcal{O}_{G}} \mathcal{O}_{\mathcal{E}}$, where $S(X, \mathcal{G})$ is the group of continuous sections of the spectral bundle $\mathcal{G} \rightarrow X$. The map $\mathcal{G} \mapsto \widehat{G}$ assigning the dual $\widehat{G}$ to the spectral bundle $\mathcal{G}$ (called the duality map) is injective.

Proof. By previous lemma it is clear that $S(X, \mathcal{G}) \subseteq$ aut $_{\mathcal{O}_{G}} \mathcal{O}_{\mathcal{E}}$. Viceversa, let $\alpha \in \operatorname{aut}_{\mathcal{O}_{G}} \mathcal{O}_{\mathcal{E}}$. Then for $\psi, \psi^{\prime} \in \widehat{\mathcal{E}}$ we find for every $y \in \mathcal{O}_{G}$ that $\psi^{*} \alpha(\psi) y=$ $\psi^{*} \alpha(\psi y)=\psi^{*} \sigma(y) \alpha(\psi)=y \psi^{*} \alpha(\psi)$, so that by Prop. 3.1 $\psi^{*} \alpha(\psi)$ belongs to $C(X)$ and $\alpha=\widehat{g}$ for some $g \in \mathbf{U} \mathcal{E}$. In order to prove that $g \in S(X, \mathcal{G})$ it suffices to verify for each $x \in X$ that $g_{x}$ belongs to the fibre $G_{x}$. But this fact is obvious, since $\widehat{g}_{x}$ is by definition the identity on elements of $\left(\mathcal{O}_{G}\right)_{x}$. Injectivity of duality map follows by the fact that each fibre $G_{x}=\operatorname{aut}_{\left(\mathcal{O}_{G}\right)_{x}} \mathcal{O}_{d}$ is uniquely determined by properties of $\mathcal{O}_{G}$, i.e. $\widehat{G}$ (while topology of $\mathcal{G}$ is fixed by the unitary bundle of $\mathcal{E}$ ).

Corollary 3.7. Let $G, G^{\prime} \subseteq \mathbf{U} \mathcal{E}$ with $G=S(X, \mathcal{G}), G^{\prime}=S\left(X, \mathcal{G}^{\prime}\right)$. Suppose there exists an automorphism $\alpha$ of $\mathcal{O}_{\mathcal{E}}$ with $\alpha\left(\sigma^{r}, \sigma^{s}\right)=\left(\sigma^{r}, \sigma^{s}\right)$ for $r, s \in \mathbb{N}$, and such that $\alpha\left(\mathcal{O}_{G}\right)=\mathcal{O}_{G^{\prime}}$. Then $G$ and $G^{\prime}$ are conjugate, i.e. there exists $u \in \mathbf{U E}$ such that $G^{\prime}=u G u^{*}$.

Proof. As $\alpha\left(\sigma^{r}, \sigma^{s}\right)=\left(\sigma^{r}, \sigma^{s}\right)$ we find that $\alpha=\widehat{u}$, where $u \in \mathbf{U} \mathcal{E}$. Let now $\widehat{g} \in \operatorname{aut}_{\mathcal{O}_{G}} \mathcal{O}_{\mathcal{E}} \simeq G, \psi \in(\iota, \mathcal{E})_{G^{\prime}}$. Then $u g u^{*} \psi=\psi\left(\right.$ as $\alpha^{-1}(\psi)=u^{*} \psi \in$ $\left.(\iota, \mathcal{E})_{G}\right)$, and by the same argument $\left(u g u^{*}\right)^{\Upsilon}$ is the identity on $\left(\mathcal{E}^{r}, \mathcal{E}^{s}\right)_{G^{\prime}}$. Thus $\left(u g u^{*}\right)^{\top} \in$ aut $_{\mathcal{O}_{G^{\prime}}} \mathcal{O}_{\mathcal{E}} \simeq G^{\prime}$. By exchanging the role of $G, G^{\prime}$ we obtain the desired equality $G^{\prime}=u G u^{*}$.

The fact that we recover $S(X, \mathcal{G})$ instead of $G$ is an example of absence of Galois property remarked in [5]. A crucial point to get injectivity of duality map $\mathcal{G} \mapsto \widehat{G}$ is that the vector bundle $\mathcal{E}$ has to be fixed; a first counterexample follows by the next lemma, implying for every vector bundle $\mathcal{E}$ an isomorphism of strict tensor $C^{*}$-categories $\widehat{\mathbf{U E}} \simeq C(X, \mathbb{U}(d))^{\Upsilon}$ (of course $\mathbf{U E}$ is not isomorphic to $C(X, \mathbb{U}(d))$ in general). More significant counterexamples will be constructed (see Corollary 3.11 below).

Lemma 3.8. $\mathcal{O}_{\mathbf{U E}}$ is the $\sigma$-stable $C^{*}$-subalgebra of $\mathcal{O}_{\mathcal{E}}$ generated by $\theta$ and $C(X)$, and is isomorphic to $C(X) \otimes \mathcal{O}_{\mathbb{U}(d)}$.

Proof. By [16], Lemma 3.6 it follows that $C(X) \otimes \mathcal{O}_{\mathbb{U}(d)}$ is generated by $C(X)$, the symmetry induced by the exchange operator $\theta_{d}$ on $\mathbb{C}^{d} \otimes \mathbb{C}^{d}$, and by closing w.r.t. action of the canonical endomorphism $\sigma_{d}$ on $\mathcal{O}_{\mathbb{U}(d)}$. We now use the notation $(\mathcal{A}, \rho, \varepsilon)$ to denote a triple whose elements are a $C(X)$-algebra $\mathcal{A}$ and a $C(X)$-endomorphism $\rho$ of $\mathcal{A}$, carrying weak permutation symmetry with the 
exchange operator $\varepsilon:=\varepsilon(1,1)$; arrows between such triples will denote $C(X)$ algebra morphisms intertwining endomorphisms and carrying the unitary $\varepsilon$ in to the analogue object. We now consider the triple $\left(C(X) \otimes \mathcal{O}_{\mathbb{U}(d)}, \sigma_{d}, \theta_{d}\right)$ with the local charts $\pi_{i}:\left(\left.\mathcal{O}_{\mathbf{U E}}\right|_{X_{i}},\left.\sigma\right|_{X_{i}},\left.\theta\right|_{X_{i}}\right) \rightarrow\left(C\left(X_{i}\right) \otimes \mathcal{O}_{\mathbb{U}(d)}, \sigma_{d}, \theta_{d}\right)$ induced by local charts $\left.\mathcal{E}\right|_{X_{i}} \rightarrow X \times \mathbb{C}^{d}$ of $\mathcal{E}$. To prove the lemma it suffices to show that the $\pi_{i}$ 's are compatible, i.e. that $\pi_{i} \pi_{j}^{-1}$ is the identity on the triple $\left(C\left(X_{i j}\right) \otimes\right.$ $\left.\mathcal{O}_{\mathbb{U}(d)}, \sigma_{d}, \theta_{d}\right)$. But this fact is obvious, as the local charts of $\mathcal{O}_{\mathbf{U E}}$ induced by the vector bundle $\mathcal{E}$ preserve symmetry and canonical endomorphism, that do not depend on the choice of a local basis. Thus for each $r \in \mathbb{N}$ we have the isomorphism $\left(\mathcal{E}^{r}, \mathcal{E}^{r}\right)_{\mathrm{U} \mathcal{E}} \simeq C^{*} \mathbb{P}_{r} \otimes C(X)$ (see [16, Appendix).

Corollary 3.9. Let $\mathcal{E}, \mathcal{E}^{\prime} \rightarrow X$ be vector bundles. There is an isomorphism of strict tensor $C^{*}$-categories $\widehat{\mathbf{U E}} \simeq \widehat{\mathbf{U E}^{\prime}}$ if and only if $\mathcal{E}, \mathcal{E}^{\prime}$ have the same rank.

Previous lemma implies that the $C^{*}$-algebra $\mathcal{O}_{\mathbf{U} \mathcal{E}}$ does not maintain geometrical informations about $\mathcal{E}$, except for the rank. By restricting the group we can recover some geometrical data, as for example the first Chern class in the case of $\mathbf{S U E}$. We are going to prove this fact. Let us consider the following shift endomorphism on $\mathcal{O}_{\mathbf{U E}}$, defined by the map

$$
\Lambda(y):=P \otimes y
$$

where $P$ is the totally antisymmetric projection (3.513.11) and $y \in\left(\mathcal{E}^{r}, \mathcal{E}^{r}\right) \mathbf{U E}$. Note that the relation $\Lambda(y)=P \cdot \sigma_{\mathbf{U} \mathcal{E}}^{d}(y)$ holds for $y \in \mathcal{O}_{\mathbf{U} \mathcal{E}}$, so that from a categorical point of view $\Lambda$ is a subobject of $\sigma_{\mathbf{U E}}^{d}$ (note in fact that $P \in$ $\left.\left(\sigma_{\mathbf{U} \mathcal{E}}^{d}, \sigma_{\mathbf{U} \mathcal{E}}^{d}\right)\right)$.

Lemma 3.10. $\mathcal{O}_{\mathrm{SUE}}$ is the $\sigma$-stable $C^{*}$-subalgebra of $\mathcal{O}_{\mathcal{E}}$ generated by $\theta$ and $(\iota, \lambda \mathcal{E})$, and is isomorphic to the crossed product $\mathcal{O}_{\mathbf{U E}} \rtimes_{\Lambda}^{\lambda \mathcal{E}} \mathbb{N}$.

Proof. Both $\mathcal{O}_{\mathbf{U E}} \rtimes_{\Lambda}^{\lambda \mathcal{E}} \mathbb{N}$ and $\mathcal{O}_{\mathbf{S U} \mathcal{E}}$ are generated as $C^{*}$-algebras by $\mathcal{O}_{\mathbf{U E}}$ and $(\iota, \lambda \mathcal{E})$, in such a way that $(\iota, \lambda \mathcal{E})$ induces $\Lambda$ on $\mathcal{O}_{\mathbf{U} \mathcal{E}}$. Note that if $\psi_{1}, \psi_{2} \in$ $(\iota, \lambda \mathcal{E})$ then for every $x \in \mathcal{O}_{\mathbf{U E}}$ we find $\psi_{1} y \psi_{2}^{*}=\Lambda(y) \psi_{1} \psi_{2}^{*}=\Lambda(y) f P \in \mathcal{O}_{\mathbf{U E}}$, for some $f \in C(X)$. Furthermore $\widehat{g}\left(\psi_{1}^{*} y \psi_{2}\right)=\psi_{1}^{*} y \psi_{2}$ for every $g \in \mathbf{S U E}$, and $\psi_{1}^{*} y \psi_{2} \in \mathcal{O}_{\mathbf{S U} \mathcal{E}}$. To prove the lemma it remains to verify universal property w.r.t. covariant representations $\left(\pi: \mathcal{O}_{\mathbf{U E}} \rightarrow \mathcal{L}(\mathcal{H}), \lambda \mathcal{E}_{\pi}\right)$. Now note that if $t \in\left(\mathcal{E}^{d}, \mathcal{E}^{d}\right)_{\mathbf{U} \mathcal{E}}$ and $\psi, \psi^{\prime} \in \lambda \mathcal{E}_{\pi}$ then $\psi^{*} \pi(t) \psi^{\prime}=\psi^{*} \pi(P) \pi(t) \pi(P) \psi^{\prime} \in C(X)$ (because $\left.P \cdot\left(\mathcal{E}^{d}, \mathcal{E}^{d}\right)_{\mathbf{U} \mathcal{E}} \cdot P=C(X) \cdot P\right)$. Furthermore $\psi^{\prime} \psi^{*} \in C(X) \cdot \pi(P)=$ $C(X) \cdot \pi \Lambda(1)$. Thus we can define define the representation $\Pi: \mathcal{O}_{\text {SUE }} \rightarrow \mathcal{L}(\mathcal{H})$ in such a way that $\Pi(t \psi):=\pi(t) \psi_{\pi}$, where $\psi_{\pi}$ is the image of $\psi \in(\iota, \lambda \mathcal{E})$ via the natural isomorphism $C^{*}\{(\iota, \lambda \mathcal{E})\} \simeq C^{*}\left\{\lambda \mathcal{E}_{\pi}\right\}$. Previous remarks imply that $\Pi$ extends $\pi$ as desired.

Corollary 3.11. Let $\mathcal{E}, \mathcal{E}^{\prime} \rightarrow X$ be vector bundles. There is an isomorphism of strict tensor $C^{*}$-categories $\widehat{\mathbf{S U E}} \simeq \widehat{\mathbf{S U \mathcal { E } ^ { \prime }}}$ if and only if $\mathcal{E}, \mathcal{E}^{\prime}$ have the same rank and first Chern class. 
Example 3.5. Let $X$ be a compact Hausdorff space with $H^{2}(X, \mathbb{Z})=\mathbb{Z}$, $\mathcal{E}:=\mathcal{L} \oplus \mathcal{L}^{*} \rightarrow X$ a vector bundle with $\mathcal{L}^{*}$ denoting the dual of a nontrivial line bundle $\mathcal{L} \rightarrow X$. By construction $(\mathcal{E}, \mathcal{E})$ is nontrival as a continuous field of matrix algebras (in fact $\mathcal{E}$ is not the tensor power of a trivial bundle by a line bundle). So that $\mathbf{S U E}$ is nontrivial (in fact $(\mathcal{E}, \mathcal{E})$ is generated as a $C^{*}$-algebra by $\left.\mathbf{U} \mathcal{E} \simeq \mathbf{S U E} \times_{X} \mathbb{T}\right)$, i.e. $\mathbf{S U E} \neq C(X, \mathbb{S U}(2))$. Despite that, by previous corollary there is an isomorphism of strict tensor $C^{*}$-categories $\widehat{\mathrm{SUE}} \simeq C(X, \mathbb{S U}(2))$, since $\lambda \mathcal{E}=\mathcal{L} \otimes \mathcal{L}^{*} \simeq X \times \mathbb{C}$ so that the first Chern class of $\mathcal{E}$ vanishes and $(\iota, \lambda \mathcal{E}) \simeq C(X) \cdot R$, where $R$ is defined by (3.2). Thus $\mathcal{O}_{\mathrm{SU} \mathcal{E}} \simeq C(X) \otimes \mathcal{O}_{\mathbb{S U}(2)}$.

Previous example shows how injectivity of duality map (Prop. 3.6) is verified only if embedding into the category of vector bundles is fixed. In fact here $\widehat{\mathbf{S U E}}$ is exhibited as a dual of not isomorphic group bundles, spanned respectively by the nontrivial $\mathbf{S U E} \simeq \operatorname{aut}_{\mathcal{O}_{\mathrm{SU}} \mathcal{\mathcal { E }}} \mathcal{O}_{\mathcal{E}}$ and $C(X, \mathbb{S U}(2)) \simeq \operatorname{aut}_{\mathcal{O}_{\mathrm{SU} \mathcal{E}}}\left(C(X) \otimes \mathcal{O}_{2}\right)$. The fact we recover different bundles depends on the choice of embedding $\widehat{\mathbf{S U E}}$ into the category of tensor powers of $\mathcal{E}:=\mathcal{L} \oplus \mathcal{L}^{*}$ (corresponding to the inclusion $\mathcal{O}_{\text {SUE }} \hookrightarrow \mathcal{O}_{\mathcal{E}}$ ), or $X \times \mathbb{C}^{2}$ (corresponding to $\left.\mathcal{O}_{\text {SUE }} \hookrightarrow C(X) \otimes \mathcal{O}_{2}\right)$.

Anyway in general the fibres $\left\{G_{x}\right\}_{x \in X}$ of a closed group $G \subseteq \mathbf{U} \mathcal{E}$ do not depend on the choice of vector bundle $\mathcal{E}^{\prime}$ realizing the embedding $\mathcal{O}_{G} \hookrightarrow \mathcal{O}_{\mathcal{E}^{\prime}}$. Thus different embeddings of $\widehat{G}$ into the category of vector bundles correspond to different topologies over $\bigsqcup_{x} G_{x}$, and define not necessarily isomorphic group bundles.

\section{Noncommutative Pullbacks of Vector Bundles.}

Let $\mathcal{A}$ be a $C^{*}$-algebra, $\mathcal{M}$ a Hilbert $\mathcal{A}$-bimodule. We denote by $\left(\mathcal{M}^{r}, \mathcal{M}^{s}\right)$ the Banach space of bounded right $\mathcal{A}$-module operators between the tensor powers $\mathcal{M}^{r}, \mathcal{M}^{s}$, where $r, s \in \mathbb{N}$. In particular, we define $\mathcal{M}^{0}:=\mathcal{A}$; note that there is a natural isomorphism $(\mathcal{A}, \mathcal{M}) \simeq \mathcal{M}$. The category with objects the tensor powers of $\mathcal{M}$ and arrows $\left(\mathcal{M}^{r}, \mathcal{M}^{s}\right)$ is a strict semitensor $C^{*}$-category in the sense of 14]; roughly speaking, a tensor product is defined on objects, while on arrows just the operation of tensoring on the right by the identity arrow is admitted. This structure reflects the well known fact that in general it is not possible to define in a consistent way the tensor product of right $\mathcal{A}$-module operators (see for example [7]).

The Pimsner algebra $\mathcal{O}_{\mathcal{M}}$ of the Hilbert bimodule $\mathcal{M}$ (34) is thus generated by elements of the spaces $\left(\mathcal{M}^{r}, \mathcal{M}^{s}\right)$, by using the procedure exposed in [14, [18] (see also [25], 26] for a detailed study about structure of Pimsner algebras).

It is also possible to associate to $\mathcal{M}$ a strict tensor $C^{*}$-category, having again objects the tensor powers of $\mathcal{M}$, but arrows the $\mathcal{A}$-bimodule operators commuting with left $\mathcal{A}$-action, that we denote by $\mathcal{B}\left(\mathcal{M}^{r}, \mathcal{M}^{s}\right)$ (see 14 for details). The problem of tensoring such operators is in this case avoided by 
requiring commutativity w.r.t. left $\mathcal{A}$-action. Following 14, we construct the $C^{*}$-algebra $\mathcal{B}_{\mathcal{M}}$ generated by the Banach bimodules $\mathcal{B}\left(\mathcal{M}^{r}, \mathcal{M}^{s}\right)$. Because of the inclusion $\mathcal{B}\left(\mathcal{M}^{r}, \mathcal{M}^{s}\right) \hookrightarrow\left(\mathcal{M}^{r}, \mathcal{M}^{s}\right)$, there is a unital inclusion $\mathcal{B}_{\mathcal{M}} \hookrightarrow \mathcal{O}_{\mathcal{M}}$ with $\mathcal{B}\left(\mathcal{M}^{r}, \mathcal{M}^{s}\right)=\left(\mathcal{M}^{r}, \mathcal{M}^{s}\right) \cap \mathcal{A}^{\prime}$ and $\mathcal{B}_{\mathcal{M}} \subseteq \mathcal{A}^{\prime} \cap \mathcal{O}_{\mathcal{M}}$. Note that the centre of $\mathcal{A}$ is contained in $\mathcal{B}_{\mathcal{M}}$.

$\mathcal{B}_{\mathcal{M}}$ is naturally endowed of the canonical endomorphism $\tau$ defined by left tensoring $\tau(t):=1 \otimes t, t \in \mathcal{B}\left(\mathcal{M}^{r}, \mathcal{M}^{s}\right)$, where 1 is the identity map on $\mathcal{M}$. Note that in general $\tau$ cannot be extended to an endomorphism of $\mathcal{O}_{\mathcal{M}}$, and is not the identity on elements of $\mathcal{A} \cap \mathcal{A}^{\prime}$. We introduce the following notation:

$$
\mathcal{Z}^{\tau}:=\left\{f \in \mathcal{A} \cap \mathcal{A}^{\prime}=(\mathcal{A}, \mathcal{A}) \cap \mathcal{A}^{\prime} \subseteq \mathcal{B}_{\mathcal{M}}: \tau(f)=f\right\} .
$$

Next lemma allows to compute the relative commutant in $\mathcal{O}_{\mathcal{M}}$ of $\mathcal{A}$ and $\mathcal{B}_{\mathcal{M}}$ in relevant particular cases.

Lemma 4.1. Let $\mathcal{M}$ be a Hilbert $\mathcal{A}$-bimodule, $\mathcal{B} \subseteq \mathcal{B}_{\mathcal{M}}$ be a $\tau$-stable, $\mathbb{Z}$ graded $C^{*}$-algebra. If for some $d \in \mathbb{N}$ there is an isometry $R \in \mathcal{B}\left(\mathcal{A}, \mathcal{M}^{d}\right) \cap \mathcal{B}$, then $\mathcal{B}_{\mathcal{M}}=\mathcal{A}^{\prime} \cap \mathcal{O}_{\mathcal{M}}$. Furthermore, if for some $\lambda \in(-1,0) \cup(0,1)$ the equality $R^{*} \tau(R)=\lambda 1$ holds then $\mathcal{A}=\mathcal{B}^{\prime} \cap \mathcal{O}_{\mathcal{M}}$ (and $\mathcal{A}$ is normal in $\mathcal{O}_{\mathcal{M}}$ in the sense of [14]).

Proof. First statement is proved in [14, Prop.3.4. Second statement follows by Prop.3.5 in the same paper by defining the sequence

$$
R_{k}:=\tau^{k-1}(R) \cdots \tau(R) R
$$

Remark 4.1. If $\mathcal{A} \simeq C(X)$ is abelian then $\mathcal{B}_{\mathcal{M}}$ is a continuous field of $C^{*}$ algebras over $X$ (in fact $\mathcal{B}_{\mathcal{M}}$ is a $C(X)$-algebra and norm functions $x \mapsto$ $\left\|t_{x}\right\|$ are continuous for elements $t$ of $\mathcal{B}\left(\mathcal{M}^{r}, \mathcal{M}^{s}\right)$ that generate $\mathcal{B}_{\mathcal{M}}$ as a $C^{*}$ algebra). $\quad \mathcal{B}_{\mathcal{M}}$ is a subfield of the $C^{*}$-algebra of the vector bundle $\mathcal{E}_{\mathcal{M}} \rightarrow$ $X$ having module of sections isomorphic to $\mathcal{M}$ as a right module (so called abelianization of $\mathcal{M}$ as in [1]). Note that the inclusion $\mathcal{B}_{\mathcal{M}} \hookrightarrow \mathcal{O}_{\mathcal{E}_{\mathcal{M}}}$ does not preserve canonical endomorphism in general, unless $\tau(a)=a$ for $a \in C(X)$.

As remarked in [5], a well defined symmetry in the sense of Doplicher and Roberts fails to exist in general for the $C^{*}$-category of tensor powers of a Hilbert bimodule. An aspect of this fact is that the exchange operator

$$
\theta\left(\psi \otimes \psi^{\prime}\right):=\psi^{\prime} \otimes \psi
$$

where $\psi, \psi^{\prime} \in \mathcal{M}$, is not a right $\mathcal{A}$-module map, unless left $\mathcal{A}$-module action coincides with the right one (i.e. $a \psi=\psi a$ for $a \in \mathcal{A}, \psi \in \mathcal{M}$ ). Anyway in general for every $r, s \in \mathbb{N}$ the map

$$
t \otimes t^{\prime} \mapsto t^{\prime} \otimes t=t^{\prime} \tau^{r^{\prime}}(t)
$$


where $t \in \mathcal{B}\left(\mathcal{M}^{r}, \mathcal{M}^{s}\right), t^{\prime} \in \mathcal{B}\left(\mathcal{M}^{r^{\prime}}, \mathcal{M}^{s^{\prime}}\right)$, is well defined as a right $\mathcal{A}$-module map. To get actually a symmetry we need to implement the maps (4.2) by using the exchange operators $\theta(r, s)$ defined as in previous section. By above considerations it is clear that the $\theta(r, s)$ 's in general are not well defined in the Pimsner algebra. We now introduce a class of Hilbert bimodules such that corresponding $C^{*}$-category of tensor powers admits a symmetric subcategory.

Definition 4.2. Let $\mathcal{M}$ be a Hilbert $\mathcal{A}$-bimodule. The canonical endomorphism $\tau$ of $\mathcal{B}_{\mathcal{M}}$ is inner w.r.t. $\mathcal{O}_{\mathcal{M}}$ if there is a finitely generated $\mathcal{Z}^{\tau}$-bimodule $\widehat{\mathcal{E}} \subset$ $(\mathcal{A}, \mathcal{M})$ with support 1 (where $\mathcal{Z}^{\tau}$ is defined by 4.1)).

It is clear that if $\tau$ is inner w.r.t. $\mathcal{O}_{\mathcal{M}}$ then $\mathcal{M}$ is generated as a right Hilbert $\mathcal{A}$-module by $\widehat{\mathcal{E}}$. Note that if $f \in \mathcal{Z}^{\tau}$ then $f \psi=\tau(f) \psi=\psi f$ for every $\psi \in \mathcal{M}$. Not also that by Serre-Swan theorem $\widehat{\mathcal{E}}$ is the module of continuous sections of a vector bundle $\mathcal{E} \rightarrow \widehat{\mathcal{Z}}^{\tau}$. We summarize in the following lemma basic properties of the Hilbert bimodules above introduced.

Lemma 4.3. Let $\mathcal{M}$ be a Hilbert $\mathcal{A}$-bimodule having the canonical endomorphism $\tau$ inner w.r.t. $\mathcal{O}_{\mathcal{M}}$. Then the following properties hold:

- $\mathcal{Z}^{\tau}$ is contained in the centre of $\mathcal{O}_{\mathcal{M}}$;

- $\widehat{\mathcal{E}}$ induces an inner endomorphism $\sigma_{\mathcal{E}}$ on $\mathcal{O}_{\mathcal{M}}$;

- $\tau=\left.\sigma_{\mathcal{E}}\right|_{\mathcal{B}_{\mathcal{M}}}$;

- $\widehat{\sigma_{\mathcal{E}}} \subset \mathbf{e n d} \mathcal{O}_{\mathcal{M}}$ is a symmetric strict tensor $C^{*}$-category;

- For every $r, s \in \mathbb{N}$ there is an inclusion $\left(\sigma_{\mathcal{E}}^{r}, \sigma_{\mathcal{E}}^{s}\right) \subseteq\left(\mathcal{M}^{r}, \mathcal{M}^{s}\right)$, i.e. $\widehat{\sigma_{\mathcal{E}}}$ is a subcategory of the one of tensor powers of $\mathcal{M}$.

Proof. First point follows by the fact that elements of $\mathcal{Z}^{\tau}$ commute with elements of $\widehat{\mathcal{E}}, \mathcal{A}$ generating $\mathcal{O}_{\mathcal{M}}$. Thus second point follows trivially by the fact that $\widehat{\mathcal{E}}$ is a finitely generated $\mathcal{Z}^{\tau}$-bimodule in $\mathcal{O}_{\mathcal{M}}$. Third point follows by the identity $(1 \otimes t) \cdot(\psi \otimes 1 \cdots \otimes 1) \cdot \psi^{\prime}=\psi \otimes t \psi^{\prime}$, where $t \in \mathcal{B}\left(\mathcal{M}^{r}, \mathcal{M}^{s}\right)$, $\psi \in(\iota, \mathcal{M}), \psi^{\prime} \in\left(\iota, \mathcal{M}^{r}\right)$, that regarded in $\mathcal{O}_{\mathcal{M}}$ becomes $(1 \otimes t) \cdot \psi=\psi \cdot t$. Fourth point follows by the fact that canonical endomorphisms of $C^{*}$-algebras of vector bundles have permutation symmetry (as proved in Sec. 3). Fifth point follows by amenability $\left(\sigma_{\mathcal{E}}^{r}, \sigma_{\mathcal{E}}^{s}\right)=\left(\mathcal{E}^{r}, \mathcal{E}^{s}\right) \hookrightarrow\left(\mathcal{M}^{r}, \mathcal{M}^{s}\right)$.

We now characterize the property of $\tau$ to be inner w.r.t. $\mathcal{O}_{\mathcal{M}}$ in terms of properties of the Hilbert bimodule $\mathcal{M}$.

Definition 4.4. Let $\mathcal{A}$ be a $C(X)$-algebra. A Hilbert $\mathcal{A}$-bimodule $\mathcal{M}$ is called noncommutative pullback of a vector bundle $\mathcal{E} \rightarrow X$ if there is an isomorphism of right Hilbert $\mathcal{A}$-modules $\mathcal{M} \simeq \widehat{\mathcal{E}} \otimes_{C(X)} \mathcal{A}$, and $f \psi=\psi f$ for $f \in C(X)$, $\psi \in \mathcal{M}$. 
The terminology is justified by usual pullbacks of vector bundles, induced by suriettive maps $X^{\prime} \rightarrow X$. A noncommutative pullback $\mathcal{M}$ naturally defines a cycle in $K K_{X}(\mathcal{A}, \mathcal{A})$ (in the sense of [27]), and admits a systems of generators $\left\{\psi_{l}\right\}$, where the $\psi_{l}$ 's are continuous sections of $\mathcal{E}$ generating $\mathcal{M}$ as a right Hilbert $\mathcal{A}$-module. Note that by using the formalism of amplimorphisms [18, noncommutative pullbacks are associated to $C^{*}$-algebra morphisms of the type $\phi: \mathcal{A} \rightarrow \mathcal{A} \otimes \mathbb{M}_{d}$ such that $\phi(1) \in C(X) \otimes \mathbb{M}_{d}$ and $\phi(f)=\phi(1) f$ for $f \in C(X)$. In such a case the corresponding module is recovered as $\mathcal{M}:=\left\{\psi \in \mathbb{C}^{d} \otimes \mathcal{A}: \phi(1) \psi=\psi\right\}$, with right $\mathcal{A}$-action given by scalar multiplication and left $\mathcal{A}$-action defined by $a, \psi \mapsto \phi(a) \psi$.

Example 4.1. Let $\mathcal{M}$ be a Hilbert $\mathcal{A}$-bimodule isomorphic as a right module to $\mathbb{C}^{d} \otimes \mathcal{A}$. Let furthermore $C(X):=\left\{f \in M(\mathcal{A}) \cap M(\mathcal{A})^{\prime}: f \psi=\psi f, \psi \in \mathcal{M}\right\}$. Then $\mathcal{M}$ is a noncommutative pullback of the trivial rank $d$ vector bundle over $X$. Bimodules of this type appear in crossed product $C^{*}$-algebras as in [5], [6]. Other examples, given by Hilbert bimodules associated to contractions in compact metric spaces, can be found in [35], $\S 4$.

Example 4.2. Let $p: Y \rightarrow X$ be a continuous surjective map of compact Hausdorff spaces (so that there is an inclusion $C(X) \hookrightarrow C(Y)$ ), $\mathcal{E} \rightarrow X$ a vector bundle with associated projection $E \in \mathbb{M}_{d} \otimes C(X)$. For every $C(X)$ automorphism $\alpha \in \operatorname{aut}_{X} C(Y)$ we consider the crossed product $\mathcal{A}:=C(Y) \rtimes_{\alpha} \mathbb{Z}$ with $\alpha(\cdot)=\operatorname{ad}_{u}(\cdot), u \in \mathcal{A}$ unitary. Then the amplimorphism $\phi_{\alpha}: \mathcal{A} \rightarrow \mathcal{A} \otimes \mathbb{M}_{d}$, $\phi_{\alpha}(a):=\operatorname{ad}_{u}(a) \cdot E$ defines a noncommutative pullback of $\mathcal{E}$. In a similar way, we can consider an endomorphism $\rho \in$ end $_{X} C(Y)$ with the noncommutative pullback $\mathcal{M}:=\widehat{\mathcal{E}} \otimes_{C(X)} C(Y)$ carrying the left $C(Y)$-action $z, m \mapsto \rho(z) \cdot m$.

Example 4.3. Let $S X$ be the suspension of a compact Hausdorff space $X$. Elements of the $C^{*}$-algebra $C(S X)$ of continuous maps over $S X$ are of the type $(x, t) \mapsto z(x, t)$, where $x \in X, t \in[-1,1]$, with $z(x, 1), z(x,-1)$ assuming constant values. The immersion $i: C(X) \hookrightarrow C(S X)$ is realized as $(i f)(x, t):=$ $f(x)$. We now consider a vector bundle $\mathcal{E} \rightarrow X$ with associated projection $E \in \mathbb{M}_{d} \otimes C(X)$. We fix $k \in \mathbb{N}, k=1, \ldots, d$ and define the amplimorphism

$$
\left[\phi_{k}(z)\right](x, t):=z\left(x, \sin ^{2 k-1} \frac{\pi t}{2}\right) \cdot E(x)
$$

$z \in C(S X)$, corresponding to a noncommutative pullback $\mathcal{M}_{k}$ of $\mathcal{E}$. Note that $\left[g, \phi_{k}(z)\right]=0$ for $g \in \mathbf{U} \mathcal{E}, z \in C(S X)$, so that $\mathbf{U} \mathcal{E}$ acts on $\mathcal{M}_{k}$ by bimodule maps (commuting with left $C(S X)$-action). It is clear that we can generalize by considering every continuous map $F:[-1,1] \rightarrow[-1,1]$ with $F(1)=1$, $F(-1)=-1$ instead of $t \mapsto \sin ^{2 k-1} \frac{\pi t}{2}$.

Example 4.4. Let $\mathcal{A}$ be a $C^{*}$-algebra with identity $1_{\mathcal{A}}, E \in C(X) \otimes \mathbb{M}_{d}$ a projection defining a vector bundle $\mathcal{E} \rightarrow X$. Denote by $\mathcal{B}$ the corner $(E \otimes$ $\left.1_{\mathcal{A}}\right) \cdot\left(C(X) \otimes \mathbb{M}_{d} \otimes \mathcal{A}\right) \cdot\left(E \otimes 1_{\mathcal{A}}\right)$. Then every unital $C(X)$-morphism $\phi$ : $C(X) \otimes \mathcal{A} \rightarrow \mathcal{B}$ corresponds to a noncommutative pullback of $\mathcal{E}$, isomorphic to 
the external tensor product $\widehat{\mathcal{E}} \otimes \mathcal{A}$ as a right Hilbert $(C(X) \otimes \mathcal{A})$-module, and carrying a natural structure of vector $\mathcal{A}$-bundle in the sense of [29].

Proposition 4.5. Let $\mathcal{A}$ be a unital $C^{*}$-algebra, $\mathcal{M}$ a Hilbert $\mathcal{A}$-bimodule. Then $\mathcal{M}$ is a noncommutative pullback if and only if the canonical endomorphism $\tau$ of $\mathcal{B}_{\mathcal{M}}$ is inner w.r.t. $\mathcal{O}_{\mathcal{M}}$.

Proof. It suffice to identify $C(X)=\mathcal{Z}^{\tau}$ to pass from a definition to the other.

Next lemma (generalizing second statement of Lemma 4.1) will be used in the sequel, and allows to compute relative commutant of certain $\tau$-stable, $\mathbb{Z}$ graded subalgebras of $\mathcal{B}_{\mathcal{M}}$.

Lemma 4.6. Let $\mathcal{M} \simeq \widehat{\mathcal{E}} \otimes_{C(X)} \mathcal{A}$ be a noncommutative pullback, $\mathcal{B} \subseteq \mathcal{B}_{\mathcal{M}}$ a $\tau$-stable, $\mathbb{Z}$-graded $C^{*}$-algebra. Suppose for some $d \in \mathbb{N}$ there is a Hilbert $C(X)$-bimodule $\mathcal{R} \subseteq\left(\iota, \mathcal{E}^{d}\right) \cap \mathcal{B}$ with $\mathcal{R}^{*}=C(X) \cdot p_{\mathcal{R}}$ (here $p_{\mathcal{R}}$ denotes the support of $\mathcal{R})$, and such that for some $\lambda \in(-1,0) \cup(0,1)$ the equality $R^{*} \tau\left(R^{\prime}\right)=\lambda R^{*} R^{\prime}$ holds for every $R, R^{\prime} \in \mathcal{R}$. Then $\mathcal{A}=\mathcal{B}^{\prime} \cap \mathcal{O}_{\mathcal{M}}$.

Proof. Hypothesis $\mathcal{R}^{*}=C(X)$ and Serre-Swan Theorem imply that $\mathcal{R}$ is the module of continuous sections of some line bundle $\overline{\mathcal{R}} \subset \mathcal{E}^{d}$. Let us consider an open cover $\left\{X_{i}\right\}$ trivializing $\mathcal{E}, \overline{\mathcal{R}}$. Being $\mathcal{A}$ a $C(X)$-algebra it has the structure of an upper semicontinuous field over $X$, so that it makes sense to consider the restrictions $\mathcal{A}_{i}$ of $\mathcal{A}$ over the closed sets $\bar{X}_{i}$. The natural epimorphism $\mathcal{A} \rightarrow \mathcal{A}_{i}$ induces a map $\mathcal{M} \rightarrow \mathcal{M}_{i}:=\mathcal{M} \otimes_{\mathcal{A}} \mathcal{A}_{i}$ of Hilbert $C^{*}$-bimodules, that by universality extends to a $C^{*}$-algebra morphism $\pi_{i}: \mathcal{O}_{\mathcal{M}} \rightarrow \mathcal{O}_{\mathcal{M}_{i}} . \pi_{i}$ is just the restriction over $\bar{X}_{i}$ of $\mathcal{O}_{\mathcal{M}}$ regarded as a field over $X$. Note that $\mathcal{M}_{i}$ is isomorphic as a right Hilbert module to $\mathbb{C}^{d} \otimes \mathcal{A}_{i}$. We denote by $R_{i}$ the isometry generating the free module of continuous sections of the restriction of $\overline{\mathcal{R}}$ to $X_{i}$. Note that by Tietze theorem for vector bundles [3] $R_{i}$ is the restriction to $X_{i}$ of some section in $\mathcal{R}$. Let now $t \in \mathcal{B}^{\prime} \cap \mathcal{O}_{\mathcal{M}}$; then the restriction $t_{i} \in \mathcal{O}_{\mathcal{M}_{i}}$ over $\bar{X}_{i}$ commutes with elements of the sequence $\left\{R_{i, k}\right\}$ defined by using $R_{i}$ as in Lemma 4.1] so that $t_{i} \in \mathcal{A}_{i}$. Thus we deduce that $t \in \mathcal{A}$.

We now pass to consider group actions over a Hilbert bimodule $\mathcal{M}$ and the associated Pimsner algebra. We denote by $\mathcal{U M}$ the group of unitary right $\mathcal{A}$ module operators of $\mathcal{M}$. Now, the group $\mathcal{B U} \mathcal{M}$ of $\mathcal{A}$-bimodule unitaries of $\mathcal{M}$ acts by automorphisms on $\mathcal{O}_{\mathcal{M}}$

$$
u \mapsto \widehat{u} \in \operatorname{aut} \mathcal{O}_{\mathcal{M}}, \quad u \in \mathcal{B U M}
$$

by extending the map $\psi \mapsto u \psi, \psi \in \mathcal{M}, u \in \mathcal{B U} \mathcal{M}$ or, equivalentely, by the analogue of (3.1) for elements of $\left(\mathcal{M}^{r}, \mathcal{M}^{s}\right)$. The $C^{*}$-algebra $\mathcal{B}_{\mathcal{M}}$ is stable w.r.t. canonical $\mathcal{B U} \mathcal{M}$-action. Note that by the same argument used for the action 
(3.1), for every $u \in \mathcal{B U} \mathcal{M}$ the canonical endomorphism $\tau$ commutes with the automorphism $\widehat{u}$ induced by $u$.

Our purpose is now to study the Pimsner algebra of a noncommutative pullback $\mathcal{M} \simeq \widehat{\mathcal{E}} \otimes_{C(X)} \mathcal{A}$, with an action induced by a closed group $G \subseteq$ $\mathbf{U E}$. We know by Lemma 4.3 that $C(X)$ is contained in the centre of $\mathcal{O}_{\mathcal{M}}$, $\widehat{\mathcal{E}}$ is a Hilbert $C(X)$-module in $\mathcal{O}_{\mathcal{M}}$, and $\mathcal{O}_{\mathcal{E}}$ is embedded in $\mathcal{O}_{\mathcal{M}}$ with $\widehat{\mathcal{E}}$ inducing on $\mathcal{O}_{\mathcal{M}}$ an inner endomorphism $\sigma_{\mathcal{E}}$, which coincides with the canonical endomorphism $\sigma$ on $\mathcal{O}_{\mathcal{E}}$. If $\mathbf{U} \mathcal{E} \subseteq \mathcal{B U} \mathcal{M}$ (i.e. $G$ on $\mathcal{M}$ by $\mathcal{A}$-bimodule maps) then $G$ acts by automorphisms on $\mathcal{O}_{\mathcal{M}}$ via (4.3).

Definition 4.7. Let $\mathcal{M}$ be a Hilbert $\mathcal{A}$-bimodule, $G \subseteq \mathcal{B U M}$ a closed group. We denote by $\widehat{G}_{\mathcal{M}}$ the $C^{*}$-category with objects the tensor powers of $\mathcal{M}$ and arrows the invariant $\mathcal{A}$-bimodules

$$
\left(\mathcal{M}^{r}, \mathcal{M}^{s}\right)_{G}:=\left\{t \in\left(\mathcal{M}^{r}, \mathcal{M}^{s}\right): g^{\otimes^{s}} \cdot t=t \cdot g^{\otimes^{r}}, g \in G\right\}
$$

Note that if $\left(\mathcal{M}^{r}, \mathcal{M}^{s}\right)_{G} \subseteq \mathcal{B}\left(\mathcal{M}^{r}, \mathcal{M}^{s}\right)$ for every $r, s \in \mathbb{N}$, then $\widehat{G}_{\mathcal{M}}$ naturally inherits the tensor structure by the category of bimodule maps between tensor powers of $\mathcal{M}$.

We denote by $\mathcal{O}_{\mathcal{M}}^{G}$ the $C^{*}$-subalgebra of $\mathcal{O}_{\mathcal{M}}$ generated by invariant spaces $\left(\mathcal{M}^{r}, \mathcal{M}^{s}\right)_{G}$ 's. Since $G$-action preserves circle action and restricts to a Banach $\mathcal{A}$-bimodule $G$-action on every $\left(\mathcal{M}^{r}, \mathcal{M}^{s}\right)$ we find that $\mathcal{O}_{\mathcal{M}}^{G}$ is actually the fixed point algebra in $\mathcal{O}_{\mathcal{M}}$ w.r.t. $G$-action. Note that if $\mathcal{M} \simeq \widehat{\mathcal{E}} \otimes_{C(X)} \mathcal{A}$ is a noncommutative pullback and $G \subseteq \mathbf{U} \mathcal{E} \cap \mathcal{B U M}$ then $\mathcal{O}_{G} \subseteq \mathcal{O}_{\mathcal{M}}^{G}$, being the restriction of action (4.3) on $\mathcal{O}_{\mathcal{E}}$ given by (3.1).

Next proposition exposes some basic properties of $\mathcal{O}_{\mathcal{M}}^{G}$. We denote by the notation aut $_{\mathcal{O}_{\mathcal{M}}^{G}}\left(\mathcal{O}_{\mathcal{M}}, \sigma_{\mathcal{E}}\right)$ the group of automorphisms of $\mathcal{O}_{\mathcal{M}}$ leaving $\mathcal{O}_{\mathcal{M}}^{G}$ pointwise fixed and commuting with the inner endomorphism $\sigma_{\mathcal{E}}$.

Proposition 4.8. Let $\mathcal{Z}$ be an abelian, unital $C(X)$-algebra, $\mathcal{M} \simeq \widehat{\mathcal{E}} \otimes_{C(X)} \mathcal{Z}$ a noncommutative pullback, $G \subseteq \mathbf{S U E}$ a closed group acting on $\mathcal{M}$ by bimodule maps (i.e. $G \subseteq \mathcal{B U} \mathcal{M}$ ). If $\mathcal{O}_{\mathcal{M}}^{G} \subseteq \mathcal{B}_{\mathcal{M}}$ then the following properties hold

- $G$ acts on $\mathcal{O}_{\mathcal{M}}, \mathcal{B}_{\mathcal{M}}$ by $\mathcal{Z}$-module automorphisms.

- $\mathcal{O}_{\mathcal{M}}^{G}$ is $\tau$-stable, where $\tau$ is the canonical endomorphism on $\mathcal{B}_{\mathcal{M}}$.

- $\mathcal{O}_{\mathcal{M}}^{G} \cap \mathcal{O}_{\mathcal{M}}^{G}=\mathcal{Z}$.

- $\mathcal{O}_{\mathcal{M}}^{G}{ }^{\prime} \cap \mathcal{O}_{\mathcal{M}}=\mathcal{Z}$ (thus $\mathcal{B}_{\mathcal{M}}{ }^{\prime} \cap \mathcal{O}_{\mathcal{M}}=\mathcal{Z}$ ).

- Denote by $\tau_{G}$ the restriction of $\tau$ to $\mathcal{O}_{\mathcal{M}}^{G}$. Then

$$
\left(\mathcal{M}^{r}, \mathcal{M}^{s}\right)_{G}=\tau_{G}^{s}(\mathcal{Z}) \cdot\left(\mathcal{E}^{r}, \mathcal{E}^{s}\right)_{G}=\left(\mathcal{E}^{r}, \mathcal{E}^{s}\right)_{G} \cdot \tau_{G}^{r}(\mathcal{Z})
$$

- Amenability holds for $\widehat{G}_{\mathcal{M}}$, i.e. $\left(\mathcal{M}^{r}, \mathcal{M}^{s}\right)_{G}=\left(\tau_{G}^{r}, \tau_{G}^{s}\right)$. 
- $S(X, \mathcal{G}) \simeq \operatorname{aut}_{\mathcal{O}_{\mathcal{M}}^{G}}\left(\mathcal{O}_{\mathcal{M}}, \sigma_{\mathcal{E}}\right)$, where $S(X, \mathcal{G})$ is the group of continuous sections of the spectral bundle $\mathcal{G} \rightarrow X$ spanned by $G$.

Proof.

- Being $G \subseteq \mathbf{S U E} \cap \mathcal{B U M}$ it is clear that $G$ acts on $\mathcal{O}_{\mathcal{M}}$ and $\mathcal{B}_{\mathcal{M}}$ is $G$-stable. Furthermore by construction $\widehat{g}(z)=z$ for every $z \in \mathcal{Z}$, since $g \in G$ commutes by hypothesis with elements of $\mathcal{Z}$.

- Second point is consequence of the fact that canonical endomorphism and $\mathcal{B U} \mathcal{M}$-action commute.

- Third point follows by the immersion $\mathcal{O}_{\mathcal{M}}^{G} \hookrightarrow \mathcal{O}_{\mathcal{E}_{\mathcal{M}}}$, implying (by Lemma 3.1) that $\mathcal{O}_{\mathcal{M}}^{G} \cap \mathcal{O}_{\mathcal{M}}^{G} \subseteq \mathcal{O}_{\mathcal{M}}^{G}{ }^{\prime} \cap \mathcal{O}_{\mathcal{E}_{\mathcal{M}}}=\mathcal{Z}$.

- Fourth point follows by Lemma 4.6 by observing that the module $(\iota, \lambda \mathcal{E})$ of continuous sections of the totally antisymmetric tensor power of $\mathcal{E}$ is contained in $\mathcal{O}_{\mathcal{M}}^{G} \subseteq \mathcal{B}_{\mathcal{M}}$.

- About fifth point, let $\left\{\psi_{l}\right\}$ be a finite set of generators of $\widehat{\mathcal{E}}$. Elements of $\left(\mathcal{M}^{r}, \mathcal{M}^{s}\right)_{G}$ are of the type $t:=\sum_{L M} \psi_{L} t_{L M} \psi_{M}^{*}$, where $\psi_{L}:=$ $\psi_{l_{1}} \cdots \psi_{l_{s}} \in\left(\iota, \mathcal{E}^{s}\right) \simeq \widehat{\mathcal{E}}^{s}, \psi_{M} \in\left(\iota, \mathcal{E}^{r}\right), t_{L M}:=\psi_{L}^{*} t \psi_{M} \in \mathcal{O}_{\mathcal{M}}^{G}{ }^{\prime} \cap$ $\mathcal{O}_{\mathcal{M}}=\mathcal{Z}$. Thus $t=\sum_{L M} \tau_{G}^{s}\left(t_{L M}\right) \psi_{L} \psi_{M}^{*}$, with $\psi_{L} \psi_{M}^{*} \in\left(\sigma_{\mathcal{E}}^{r}, \sigma_{\mathcal{E}}^{s}\right)$ and $t \in \tau_{G}^{s}(\mathcal{Z}) \cdot\left(\mathcal{E}^{r}, \mathcal{E}^{s}\right)$. Let now $x \in X, \mathcal{M}_{x}:=\mathcal{M} \otimes_{C(X)} \mathcal{Z}_{x}$, where $\mathcal{Z}_{x}$ is the fibre of $\mathcal{Z}$ over $x$ as un upper semicontinuous bundle. Then $\mathcal{M}_{x} \simeq \mathcal{Z}_{x} \otimes \mathbb{C}^{d}$, and $G_{x} \subseteq \mathbb{S U}(d)$ acts on $\mathcal{O}_{\mathcal{M}_{x}}$ by action (3.1). If $t \in\left(\mathcal{M}^{r}, \mathcal{M}^{s}\right)_{G} \subseteq \tau_{G}^{s}(\mathcal{Z}) \cdot\left(\mathcal{E}^{r}, \mathcal{E}^{s}\right)$ then $t_{x}=\sum_{l} z_{l} t_{l}$, where $z_{l} \in\left(\tau_{G}^{s}(\mathcal{Z})\right)_{x}$ is $G_{x}$-invariant and $t_{l} \in \mathbb{M}_{d^{r}, d^{s}}$. Now by averaging w.r.t. Haar measure of $G_{x}$ we obtain

$$
t_{x}=\int_{G_{x}} \widehat{g}(t) d g=\sum_{l} z_{l} \int_{G_{x}} \widehat{g}\left(t_{l}\right) d g \in\left(\tau_{G}^{s}(\mathcal{Z})\right)_{x} \cdot\left(\mathcal{E}^{r}, \mathcal{E}^{s}\right)_{G_{x}}
$$

By Lemma 3.3 we obtain $\left(\mathcal{E}^{r}, \mathcal{E}^{s}\right)_{G_{x}}=\left(\left(\mathcal{E}^{r}, \mathcal{E}^{s}\right)_{G}\right)_{x}$, thus $t \in \tau_{G}^{s}(\mathcal{Z})$. $\left(\mathcal{E}^{r}, \mathcal{E}^{s}\right)_{G}$.

- Let $t \in\left(\mathcal{M}^{r}, \mathcal{M}^{s}\right)_{G}$. Then by previous point $t \in \tau_{G}^{s}(\mathcal{Z})\left(\sigma_{G}^{r}, \sigma_{G}^{s}\right) \subset$ $\left(\tau_{G}^{r}, \tau_{G}^{s}\right)$. Viceversa, if $t \in\left(\tau_{G}^{r}, \tau_{G}^{s}\right)$ then $t_{L M}$ as defined above belongs to $\mathcal{Z}$, thus $t \cdot \psi_{L} \in \mathcal{M}^{s}$ and $t \in\left(\mathcal{M}^{r}, \mathcal{M}^{s}\right)$.

- Finally, let $\alpha \in \operatorname{aut}_{\mathcal{O}_{\mathcal{M}}^{G}}\left(\mathcal{O}_{\mathcal{M}}, \sigma_{\mathcal{E}}\right)$. Then for $\psi, \psi^{\prime} \in\left(\iota, \sigma_{\mathcal{E}}\right)$ we find that $\psi^{*} \alpha\left(\psi^{\prime}\right)$ belongs to the commutant of $\mathcal{O}_{\mathcal{M}}^{G}$. Thus $\psi^{*} \alpha\left(\psi^{\prime}\right) \in \mathcal{Z}$. Now by hypothesis $\alpha \circ \sigma_{\mathcal{E}}=\sigma_{\mathcal{E}} \circ \alpha$, so that $\tau\left(\psi^{*} \alpha\left(\psi^{\prime}\right)\right)=\psi^{*} \theta \cdot \sigma_{\mathcal{E}} \circ \alpha\left(\psi^{\prime}\right)=$ $\psi^{*} \theta \alpha\left(\theta \psi^{\prime}\right)=\psi^{*} \alpha\left(\psi^{\prime}\right)$, since $\theta$ (defined as in (3.7)) belongs to $\mathcal{O}_{G}$ and is $G$-invariant. Thus we conclude that $\psi^{*} \alpha\left(\psi^{\prime}\right) \in C(X)$, and $\alpha=\widehat{g}$ for some $g \in \mathbf{U} \mathcal{E}$. Thus $\widehat{g} \in \mathbf{a u t}_{\mathcal{O}_{\mathcal{M}}^{G}}\left(\mathcal{O}_{\mathcal{M}}, \sigma_{\mathcal{E}}\right)$ restricts to an automorphism of $\mathcal{O}_{\mathcal{E}}$ leaving $\mathcal{O}_{G} \subseteq \mathcal{O}_{\mathcal{M}}^{G}$ pointwise invariant, so that we apply Prop 3.6. and conclude $S(X, \mathcal{G}) \simeq \operatorname{aut}_{\mathcal{O}_{\mathcal{M}}^{G}}\left(\mathcal{O}_{\mathcal{M}}, \sigma_{\mathcal{E}}\right)$. 


\section{Crossed Products by Dual Actions.}

Let $\mathcal{A}$ be a unital $C^{*}$-algebra with the strict tensor $C^{*}$-category of endomorphisms end $\mathcal{A}$ introduced in Sec. 1

Definition 5.1. Let $X$ be a compact Hausdorff space, $\mathcal{E} \rightarrow X$ a vector bundle with a closed group $G \subseteq \mathbf{U E}$. A dual $G$-action on $\mathcal{A}$ is an injective functor of strict tensor $C^{*}$-categories $\mu: \widehat{G} \rightarrow \operatorname{end} \mathcal{A}$.

Here $\widehat{G}$ is the dual defined in Def. 3.2 Note that since $\widehat{G}=S(X, \mathcal{G})$ we can always assume $G=S(X, \mathcal{G})$. In the sequel we will denote $\rho:=\mu(\mathcal{E})$, so that a dual $G$-action (being all the objects of $\widehat{G}$ tensor powers of $\mathcal{E}$ ) is actually an injective functor of strict tensor $C^{*}$-categories $\mu: \widehat{G} \rightarrow \widehat{\rho}$, where $X$ is chosen to coincide with the spectrum $\widehat{\mathcal{Z}}^{\rho}$ of the abelian $C^{*}$-algebra $\mathcal{Z}^{\rho}$ defined in (2.1), and $\widehat{\rho}$ is the full subcategory of end $\mathcal{A}$ generated by the powers of $\rho$. Note that both $\mathcal{O}_{G}$ and $\mathcal{A}$ have an obvious structure of $\mathcal{Z}^{\rho}$-algebras. It is now clear by amenability (i.e. $\left(\sigma_{G}^{r}, \sigma_{G}^{s}\right)=\left(\mathcal{E}^{r}, \mathcal{E}^{s}\right)_{G}$, see Prop 3.1) that a dual $G$-action $\mu$ on $(\mathcal{A}, \rho)$ can be regarded as an injective $\mathcal{Z}^{\rho}$-morphism $\mu: \mathcal{O}_{G} \rightarrow \mathcal{A}$ such that

- $\mu \circ \sigma_{G}=\rho \circ \mu$;

- $\mu\left(\sigma_{G}^{r}, \sigma_{G}^{s}\right) \subseteq\left(\rho^{r}, \rho^{s}\right), r, s=1,2, \ldots$

The definition of dual action by Doplicher and Roberts in 19 is thus encoded in our general setting. In fact given a closed Lee group $G \subseteq \mathbb{U}(d)$ and an action $\mu_{0}: \mathcal{O}_{G} \rightarrow \mathcal{A}$ in the sense of [19] we can construct the homomorphism

$$
\mu_{0} \otimes i d: \mathcal{O}_{G} \otimes \mathcal{Z}^{\rho} \rightarrow \mathcal{A}
$$

satisfying above properties equivalent to Def 5.1 In this case we regard at $\mathcal{O}_{G} \otimes \mathcal{Z}^{\rho}$ as the algebra associated to the trivial group $C\left(\widehat{\mathcal{Z}}^{\rho}, G\right)$.

Aim of the present section is to construct, given a dual action $\mu$ of $G$ on $(\mathcal{A}, \rho)$, a universal $C^{*}$-algebra $\mathcal{A} \rtimes_{\mu} \widehat{G}$ with a commutative diagram

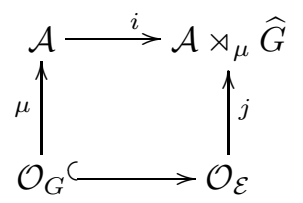

such that

$$
\psi \cdot i(a)=i \circ \rho(a) \cdot \psi
$$

where $\psi \in j(\widehat{\mathcal{E}}), a \in \mathcal{A}$. In such a case we will say that $\mathcal{A} \rtimes_{\mu} \widehat{G}$ is the crossed product of $\mathcal{A}$ by the dual action $\mu$. Note that by commutativity of above 
diagram $i(f)=j(f)$ for $f \in \mathcal{Z}^{\rho}$. As usual if $\left\{\psi_{l}\right\}$ is a set of generators for $j(\widehat{\mathcal{E}})$ then by (5.2) we find $i \circ \rho(a)=\sum_{l} \psi_{l} i(a) \psi_{l}{ }^{*}$.

Example 5.1. Let $\left(\mathcal{F}, \pi_{x}: \mathcal{F} \rightarrow \mathbb{M}_{d}\right)_{x \in X}$ be a continuous field of matrix algebras over a compact Hausdorff space $X$. We consider the $C^{*}$-algebra $\mathcal{F}_{\infty}:=$ $\bigotimes_{\mathbb{N}} \mathcal{F}$, where the tensor product has coefficients in $C(X) \cdot \mathcal{F}_{\infty}$ is a continuous field having as fibre the zero grade component $\mathcal{O}_{d}^{0}$ of the Cuntz algebra $\mathcal{O}_{d}$. $\mathcal{F}_{\infty}$ comes naturally equipped of the shift endomorphism $\sigma_{\infty} \simeq \sigma_{k}: \otimes^{k} \mathcal{F} \hookrightarrow$ $\otimes^{k+1} \mathcal{F}$, carrying $t \in \otimes^{k} \mathcal{F}$ into $1 \otimes t$, where 1 is the unit of $\mathcal{F}$. If the DixmierDouady class of $\mathcal{F}$ vanishes in $H^{3}(X, \mathbb{Z})$ then $\mathcal{F}$ is isomorphic to the continuous field of endomorphisms of some vector bundle $\mathcal{E} \rightarrow X$, defined up to tensor product by some line bundle. We now consider the circle group $\mathbb{T} \subset \mathbf{U E}$, acting on $\mathcal{E}$ by scalar multiplication. The induced $\mathbb{T}$-action on $\mathcal{O}_{\mathcal{E}}$ is the usual circle action, so that $\mathcal{O}_{\mathbb{T}}=\mathcal{O}_{\mathcal{E}}^{0}$. We denote by $\sigma_{\mathbb{T}}$ the restriction of the canonical endomorphism $\sigma$ to $\mathcal{O}_{\mathcal{E}}^{0}$. We now prove that for each vector bundle as above there is a dual $\mathbb{T}$-action $\mu_{\mathcal{E}}$ on $\left(\mathcal{F}_{\infty}, \sigma_{\infty}\right)$, and that $\mathcal{O}_{\mathcal{E}}$ is the crossed product of $\left(\mathcal{F}_{\infty}, \sigma_{\infty}\right)$ by $\mu_{\mathcal{E}}$. The first statement is an immediate consequence of the isomorphism $(\mathcal{E}, \mathcal{E}) \simeq \mathcal{F}$. As $\left(\mathcal{E}^{2}, \mathcal{E}^{2}\right) \simeq(\mathcal{E}, \mathcal{E}) \otimes_{C(X)}(\mathcal{E}, \mathcal{E})$ the following commutative diagram holds:

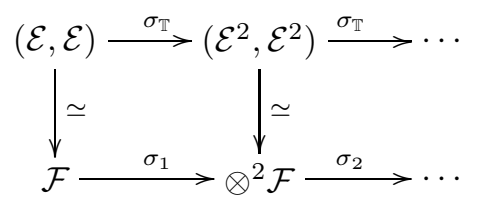

As the identifications $\left(\sigma_{\mathbb{T}}^{r}, \sigma_{\mathbb{T}}^{r}\right) \simeq\left(\mathcal{E}^{r}, \mathcal{E}^{r}\right)$ and $\left(\sigma_{\infty}^{r}, \sigma_{\infty}^{r}\right) \simeq \otimes^{r} \mathcal{F}$ hold, the above commutative diagram implies that there is an isomorphism of $C^{*}$-dynamical systems $\mu_{\mathcal{E}}:\left(\mathcal{F}_{\infty}, \sigma_{\infty}\right) \simeq\left(\mathcal{O}_{\mathcal{E}}^{0}, \sigma_{\mathbb{T}}\right) ;$ for the same reason $\mu_{\mathcal{E}}\left(\sigma_{\infty}^{r}, \sigma_{\infty}^{r}\right)=\left(\sigma_{\mathbb{T}}^{r}, \sigma_{\mathbb{T}}^{r}\right)$, so that $\mu_{\mathcal{E}}$ is a dual action. We have now to prove that $\mathcal{O}_{\mathcal{E}}$ is the crossed product of $\left(\mathcal{F}_{\infty}, \sigma_{\infty}\right)$ by $\mu_{\mathcal{E}}$ in the sense above. By the isomorphism $\left(\mathcal{F}_{\infty}, \sigma_{\infty}\right) \simeq$ $\left(\mathcal{O}_{\mathcal{E}}^{0}, \sigma_{\mathbb{T}}\right)$ follows immediately that $\sigma_{\infty}$ becomes inner in $\mathcal{O}_{\mathcal{E}}$, induced by $(\iota, \sigma) \simeq$ $\widehat{\mathcal{E}}$. Furthermore the morphisms

$$
\mathcal{F}_{\infty} \stackrel{\mu_{\mathcal{E}}}{\simeq} \mathcal{O}_{\mathcal{E}}^{0} \hookrightarrow \mathcal{O}_{\mathcal{E}}
$$

realize property (5.1), and we find $\mathcal{O}_{\mathcal{E}}=\mathcal{F}_{\infty} \rtimes_{\mu_{\mathcal{E}}} \widehat{\mathbb{T}}$. In other terms, by the isomorphism of $C^{*}$-dynamical systems $\left(\mathcal{F}_{\infty}, \sigma_{\infty}\right) \simeq\left(\mathcal{O}_{\mathcal{E}}^{0}, \sigma_{\mathbb{T}}\right)$ we obtain $\mathcal{O}_{\mathcal{E}}=$ $\mathcal{O}_{\mathcal{E}}^{0} \rtimes_{\mu_{\mathcal{E}}} \widehat{\mathbb{T}}$, but also $\mathcal{O}_{\mathcal{E} \otimes \mathcal{L}}=\mathcal{O}_{\mathcal{E}}^{0} \rtimes_{\mu_{\mathcal{E} \otimes \mathcal{L}}} \widehat{\mathbb{T}}$ for every line bundle $\mathcal{L} \rightarrow X$.

Example 5.2. Let $\mathcal{E} \rightarrow X$ be a vector bundle over a compact Hausdorff space $X, G \subseteq \mathbf{U E}$ a closed group. By amenability $\left(\mathcal{E}^{r}, \mathcal{E}^{s}\right)_{G}=\left(\sigma_{G}^{r}, \sigma_{G}^{s}\right)$ a dual action $\mu: \widehat{G} \rightarrow \widehat{\sigma}_{G} \subset$ end $\mathcal{O}_{G}$ is defined. Now the inclusion $\mathcal{O}_{G} \hookrightarrow \mathcal{O}_{\mathcal{E}}$ induces the commutative diagram (5.1), where $\mu, j$ are the identity map, and $i$ is the inclusion $\mathcal{O}_{G} \hookrightarrow \mathcal{O}_{\mathcal{E}}$. Being the canonical endomorphism inner in $\mathcal{O}_{\mathcal{E}}$ property 
(5.2) is satisfied, thus $\mathcal{O}_{\mathcal{E}}=\mathcal{O}_{G} \rtimes_{\mu} \widehat{G}$. More generally, let $\mathcal{E}, \mathcal{E}^{\prime} \rightarrow X$ be vector bundles, with $G \subseteq \mathbf{U E}, G^{\prime} \subseteq \mathbf{U} \mathcal{E}^{\prime}$ such that there is an isomorphism of symmetric strict tensor $C^{*}$-categories $\mu: \widehat{G} \rightarrow \widehat{G^{\prime}}$. Then (by amenability) $\mu$ defines a dual action $\mu: \widehat{G} \rightarrow \widehat{\sigma}_{G^{\prime}} \subset$ end $\mathcal{O}_{G^{\prime}}$ and $\mathcal{O}_{\mathcal{E}}=\mathcal{O}_{G^{\prime}} \rtimes_{\mu} \widehat{G}$. For example, if $\mathcal{E}, \mathcal{E}^{\prime}$ have the same rank and first Chern class then $\mathcal{O}_{\mathcal{E}}=\mathcal{O}_{\mathbf{S U}} \mathcal{E}^{\prime} \rtimes_{\mu} \widehat{\mathbf{S U E}}$ (see Cor 3.11).

We now proceed to the construction of our crossed product, starting at a purely *-algebraic level. We fix our notations with $a, a^{\prime}, \ldots$ for elements of $\mathcal{A}$, $f, f^{\prime}, \ldots$ for elements of $\mathcal{Z}^{\rho}, t, t^{\prime}, \ldots$ for elements of ${ }^{0} \mathcal{O}_{\mathcal{E}}, \psi, \psi^{\prime}, \ldots$ for elements of $(\iota, \mathcal{E}), y, y^{\prime}, \ldots$ for elements of ${ }^{0} \mathcal{O}_{G}$. Observe that $\mathcal{A}$ has the following structure of ${ }^{0} \mathcal{O}_{G}$-bimodule:

$$
\left\{\begin{array}{l}
y, a \mapsto y \cdot a:=\mu(y) a \\
a, y \mapsto a \cdot y:=a \mu(y)
\end{array}\right.
$$

and the same is obviously true for ${ }^{0} \mathcal{O}_{\mathcal{E}}$ with left and right multiplication by elements of ${ }^{0} \mathcal{O}_{G}$. Let us now suppose to have a solution to our problem. Then we get the relation $i(a) j(y t)=i(a \mu(y)) j(t)$, and this suggests to consider the quotient $\mathcal{A} \odot{ }_{\mu}{ }^{0} \mathcal{O}_{\mathcal{E}}$ of the algebraic tensor product $\mathcal{A} \odot{ }^{0} \mathcal{O}_{\mathcal{E}}$ by the relation

$$
a \mu(y) \otimes t-a \otimes y t
$$

providing the tensor product of ${ }^{0} \mathcal{O}_{G}$-bimodules. By construction $\mathcal{A} \odot{ }_{\mu}{ }^{0} \mathcal{O}_{\mathcal{E}}$ is a ${ }^{0} \mathcal{O}_{G}$-bimodule; in particular it is a Hilbert $\mathcal{Z}^{\rho}$-bimodule with coinciding left and right action. Universality implies that for each $C^{*}$-algebra $\mathcal{B}$ satisfying (5.15.2) there is a unique linear application $\mathcal{A} \odot_{\mu}{ }^{0} \mathcal{O}_{\mathcal{E}} \rightarrow \mathcal{B}$ defined by $a \otimes_{\mu} t \mapsto i(a) j(t)$.

Our purpose is now to define a structure of ${ }^{*}$-algebra on $\mathcal{A} \odot_{\mu}{ }^{0} \mathcal{O}_{\mathcal{E}}$. It will be convenient to consider the left regular representation of $\mathcal{A} \odot_{\mu}{ }^{0} \mathcal{O}_{\mathcal{E}}$; as a first step we define for each $a, \psi$ the following $\mathcal{Z}^{\rho}$-module endomorphisms on $\mathcal{A} \odot{ }_{\mu}{ }^{0} \mathcal{O}_{\mathcal{E}}:$

$$
\begin{gathered}
\bar{a}\left(a^{\prime} \otimes_{\mu} t\right):=a a^{\prime} \otimes_{\mu} t \\
\bar{\psi}\left(a^{\prime} \otimes_{\mu} t\right):=\rho\left(a^{\prime}\right) \otimes_{\mu} \psi t
\end{gathered}
$$

Note that the following relation holds for $\bar{\psi}, \bar{a}$ as elements of the algebra end $\left(\mathcal{A} \odot_{\mu}\right.$ ${ }^{0} \mathcal{O}_{\mathcal{E}}$ ) of $\mathcal{Z}^{\rho}$-module endomorphisms on $\mathcal{A} \odot_{\mu}{ }^{0} \mathcal{O}_{\mathcal{E}}$ :

$$
\bar{\psi} \cdot \bar{a}=\overline{\rho(a)} \cdot \bar{\psi}
$$

We now make the assumption that $G \subseteq \mathbf{S U E}$, so that $(\iota, \lambda \mathcal{E}) \subseteq\left(\iota, \mathcal{E}^{d}\right)_{G}$. We consider a set of generators $\left\{R_{i}\right\}$ of the totally antisymmetric bundle $\lambda \mathcal{E}$ and define

$$
\widehat{\psi}_{l}^{i}:=\sqrt{d} \psi_{l}^{*} R_{i} \in\left(\iota, \sigma^{d-1}\right) .
$$


Let us establish some useful relations for the $\widehat{\psi}_{l}^{i}$ 's. By definition follows immediately $\widehat{\psi}_{l}^{i *} \widehat{\psi}_{m}^{j} \in \mathcal{Z}^{\rho}$; furthermore multiplying on the left by $\psi_{l}$ and summing over $l$ in (5.7) we get

$$
\sum_{l} \psi_{l} \widehat{\psi}_{l}^{i}=\sqrt{d} R_{i}
$$

now, by definition of $\widehat{\psi}_{l}^{i}$,

$$
R_{i}^{*} \psi_{l}=\frac{1}{\sqrt{d}} \widehat{\psi}_{l}^{i *}
$$

so, by using (3.10) we find

$$
(-1)^{d-1} \frac{1}{d} \psi_{l}=\sum_{i} R_{i}^{*} \sigma\left(R_{i}\right) \psi_{l}=\sum_{i} R_{i}^{*} \psi_{l} R_{i}=\frac{1}{\sqrt{d}} \sum_{i} \widehat{\psi}_{l}^{i *} R_{i}
$$

hence

$$
\psi_{l}^{*}=\sqrt{d}(-1)^{d-1} \sum_{i} R_{i}^{*} \widehat{\psi}_{l}^{i} .
$$

Above identities suggest to define, keeping in mind (5.5) and recalling that the $R_{i}$ 's belong to ${ }^{0} \mathcal{O}_{\mathrm{SUE}} \subset{ }^{0} \mathcal{O}_{G}$,

$$
\overline{\psi_{l}^{*}}\left(a \otimes_{\mu} t\right):=\sqrt{d}(-1)^{d-1} \sum_{i} \mu\left(R_{i}^{*}\right) \rho^{d-1}(a) \otimes_{\mu} \widehat{\psi}_{l}^{i} t .
$$

By previous definition, using the fact that $\mu\left(R_{i}^{*}\right) \in\left(\rho^{d}, \iota\right)$ and by (5.9) we get

$$
\overline{\psi_{l}^{*}}\left(\rho(a) \otimes_{\mu} t\right)=a \otimes_{\mu} \psi_{l}^{*} t .
$$

Lemma 5.2. Let $\left\{\psi_{l}\right\}$ be a set of generators for $(\iota, \mathcal{E})$. The following relations hold

$$
\left\{\begin{array}{l}
\overline{f \psi}_{m}=\bar{\psi}_{m} \bar{f} \\
\bar{\psi}_{l}^{*} \cdot \bar{\psi}_{m}=\overline{\left\langle\psi_{l}, \psi_{m}\right\rangle} \\
\sum_{l} \bar{\psi}_{l} \bar{\psi}_{l}^{*}=i d
\end{array}\right.
$$

Proof. First identity follows immediately by (5.6). In order to prove the second identity we compute

$$
\begin{aligned}
\overline{\psi_{l}^{*}} \cdot \bar{\psi}_{m}\left(a \otimes_{\mu} t\right) & =\overline{\psi_{l}^{*}}\left(\rho(a) \otimes_{\mu} \psi_{m} t\right)= \\
& =a \otimes_{\mu}(-1)^{d-1} \sqrt{d} \sum_{i} R_{i}^{*} \widehat{\psi}_{l}^{i} \psi_{m} t= \\
& =a \otimes_{\mu} \psi_{l}^{*} \psi_{m} t ;
\end{aligned}
$$

about the third one 


$$
\begin{aligned}
\sum_{l} \bar{\psi}_{l} \bar{\psi}_{l}^{*}\left(a \otimes_{\mu} t\right) & =(-1)^{d-1} \sqrt{d} \sum_{l, i} \bar{\psi}_{l}\left(\mu\left(R_{i}^{*}\right) \rho^{d-1}(a) \otimes_{\mu} \widehat{\psi}_{l}^{i} t\right)= \\
& =(-1)^{d-1} \sqrt{d} \sum_{l, i} \rho \circ \mu\left(R_{i}^{*}\right) \rho^{d}(a) \otimes_{\mu} \psi_{l} \widehat{\psi}_{l}^{i} t= \\
& =(-1)^{d-1} d \sum_{i} \mu \circ \sigma\left(R_{i}^{*}\right) \mu\left(R_{i}\right) a \otimes_{\mu} t= \\
& =a \otimes_{\mu} t .
\end{aligned}
$$

By characterization of ${ }^{0} \mathcal{O}_{\mathcal{E}}$ in terms of generators and relations (see [40]), and previous lemma we obtain

Corollary 5.3. The map $\psi \mapsto \bar{\psi}, \psi \in(\iota, \mathcal{E})$, is a $\mathcal{Z}^{\rho}$-module map and extends to a ${ }^{*}$-monomorphism from ${ }^{0} \mathcal{O}_{\mathcal{E}}$ into end $\left(\mathcal{A} \odot \odot_{\mu}{ }^{0} \mathcal{O}_{\mathcal{E}}\right)$.

Lemma 5.4. If $y \in{ }^{0} \mathcal{O}_{G}$ then $\bar{y}=\overline{\mu(y)}$.

Proof. We define $\mathcal{F}_{\mu}:=\left\{y \in{ }^{0} \mathcal{O}_{G}: \bar{y}=\overline{\mu(y)}\right\}$; the lemma will be proved if we verify that $\mathcal{F}_{\mu}={ }^{0} \mathcal{O}_{G} . \mathcal{F}_{\mu}$ is obviously a subalgebra of ${ }^{0} \mathcal{O}_{G}$, as - by the previous corollary - the map $y \mapsto \bar{y}$ is multiplicative on ${ }^{0} \mathcal{O}_{\mathcal{E}} \supset{ }^{0} \mathcal{O}_{G}$. As a first step we compute

$$
\begin{aligned}
\overline{\sigma_{G}(y)} & =\sum_{l} \bar{\psi} \bar{y}_{l}^{*}=\sum_{l} \bar{\psi}_{l} \overline{\mu(y)} \bar{\psi}_{l}^{*}= \\
& =\overline{\rho \circ \mu(y)} \sum_{l} \bar{\psi}_{l} \bar{\psi}_{l}^{*}=\overline{\rho \circ \mu(y)}=\overline{\mu \circ \sigma_{G}(y)}
\end{aligned}
$$

so that $\mathcal{F}_{\mu}$ is $\sigma_{G}$-stable. Then we verify that $\left(\iota, \mathcal{E}^{r}\right)_{G} \subset \mathcal{F}_{\mu}$ for each $r$; at this purpose observe that if $y \in\left(\iota, \mathcal{E}^{r}\right)_{G}$ then $\mu(y) \in\left(\iota, \rho^{r}\right)$, so

$$
\bar{y}\left(a \otimes_{\mu} t\right)=\rho^{r}(a) \otimes_{\mu} y t=\rho^{r}(a) \mu(y) \otimes_{\mu} t=\mu(y) a \otimes_{\mu} t=\overline{\mu(y)}\left(a \otimes_{\mu} t\right) ;
$$

hence $\left(\iota, \mathcal{E}^{r}\right)_{G} \subset \mathcal{F}_{\mu}$ and in particular, as $G \subset \mathbf{S U E},(\iota, \lambda \mathcal{E}) \subset \mathcal{F}_{\mu}$. The next step is to verify that $\theta \in \mathcal{F}_{\mu}$. At this purpose recall (3.7) and compute

$$
\begin{aligned}
\bar{\psi}_{l}^{*} \bar{\psi}_{m}^{*}\left(a \otimes_{\mu} t\right) & =(-1)^{d-1} d \sum_{j} \bar{\psi}_{m}^{*}\left(\mu\left(R_{j}^{*}\right) \rho^{d-1}(a) \otimes_{\mu} \widehat{\psi}_{m}^{j} t\right)= \\
& =d \sum_{i, j} \mu\left(R_{i}^{*}\right) \rho^{d-1}\left(\mu\left(R_{j}^{*}\right) \rho^{d-1}(a)\right) \otimes_{\mu} \widehat{\psi}_{l}^{i} \widehat{\psi}_{m}^{j} t= \\
& =d \sum_{i, j} \mu\left(R_{i}\right)^{*} \rho^{d-1}\left(\mu\left(R_{j}\right)^{*} \rho^{2 d-2}(a)\right) \otimes_{\mu} \widehat{\psi}_{l}^{i} \widehat{\psi}_{m}^{j} t
\end{aligned}
$$

so, by using (5.8), $\rho \circ \mu\left(R_{i}\right)=\mu \circ \sigma_{G}\left(R_{i}\right) \in\left(\rho, \rho^{d+1}\right)$ and (3.10) 


$$
\begin{aligned}
\bar{\theta}\left(a \otimes_{\mu} t\right) & =\sum_{l, m} \overline{\psi_{l} \psi_{m} \psi_{l}^{*} \psi_{m}^{*}}\left(a \otimes_{\mu} t\right)= \\
& =d \bar{\psi}_{l} \sum_{i, j, l, m} \rho \circ \mu\left(R_{i}^{*}\right) \rho^{2 d-1}(b) \otimes_{\mu} \psi_{m} \widehat{\psi}_{l}^{i} \widehat{\psi}_{m}^{j} t= \\
& =d^{2} \sum_{i, j} \mu(\theta) \rho^{2} \circ \mu\left(R_{i}^{*}\right) \rho^{d+1} \circ \mu\left(R_{j}^{*}\right) \rho^{2 d}(a) \otimes_{\mu} \sigma_{G}\left(R_{i}\right) R_{j} t= \\
& =d^{2} \mu(\theta)\left(\sum_{i, j} \rho \circ \mu\left(\sigma_{G}\left(R_{i}^{*}\right) R_{i}\right) \mu\left(\sigma_{G}\left(R_{j}^{*}\right) R_{j}\right)\right) a \otimes_{\mu} t= \\
& =\mu(\theta) a \otimes_{\mu} t=\overline{\mu(\theta)}\left(a \otimes_{\mu} t\right) .
\end{aligned}
$$

We proved in this way that $\theta$ belongs to $\mathcal{F}_{\mu}$. To prove that for each $i R_{i} R_{i}^{*}$ belongs to $\mathcal{F}_{\mu}$ recall that $P$ as defined in (3.5) belongs to the $\sigma_{G}$-stable algebra generated by $\theta$. Now we have proved that $\mathcal{F}_{\mu}$ is a $\sigma_{G}$-stable algebra and contains $\theta$, so $P \in \mathcal{F}_{\mu}$ and this implies that each $R_{i} R_{i}^{*} \in \mathcal{Z}^{\rho} P$ belongs to $\mathcal{F}_{\mu}$ (as in particular $\mathcal{Z}^{\rho} \subset \mathcal{F}_{\mu}$ ). To prove now that each $R_{i}^{*}$ belongs to $\mathcal{F}_{\mu}$ note that $R_{i} R_{i}^{*} R_{i}=\lambda_{i}^{2} R_{i}$ (where $\lambda_{i}^{2}:=R_{i}^{*} R_{i} \in \mathcal{Z}^{\rho}$ ) and compute

$$
\lambda_{i}^{2} \bar{R}_{i}^{*}=\overline{R_{i}^{*} R_{i} R_{i}^{*}}=\bar{R}_{i}^{*} \overline{\mu\left(R_{i} R_{i}^{*}\right)}=\bar{R}_{i}^{*} \overline{\mu\left(R_{i}\right) \mu\left(R_{i}^{*}\right)}=\bar{R}_{i}^{*} \bar{R}_{i} \overline{\mu\left(R_{i}^{*}\right)}=\lambda_{i}^{2} \overline{\mu\left(R_{i}^{*}\right)}
$$

so, having each $R_{i}$ the same support of $\lambda_{i}$, we conclude that $R_{i}^{*} \in \mathcal{F}_{\mu}$. Let finally $y \in\left(\mathcal{E}^{r}, \mathcal{E}^{s}\right)_{G}$. We use the notation $\psi_{L}:=\psi_{l_{1}} \ldots \psi_{l_{r}}$, where $\left\{\psi_{l}\right\}$ is a set of generators of $(\iota, \mathcal{E})$. Then $y$ is a linear combination with coefficients in $\mathcal{Z}^{\rho}$ of terms of the type $\psi_{L} \psi_{M}^{*}$; by using (5.9) and the fact that $R_{i}^{*} \in\left(\sigma_{G}^{d}, \iota\right), \widehat{\psi}_{l}^{i} \in$ $\left(\iota, \sigma^{d-1}\right)$ we get

$$
\psi_{L} \psi_{M}^{*}=\sum_{J} \sigma_{G}^{r}\left(R_{j_{1}}^{*} \sigma_{G}^{d-1}\left(R_{j_{2}}^{*}\right) \ldots \sigma_{G}^{(s-1)(d-1)}\left(R_{j_{s}}^{*}\right)\right) \psi_{L} \widehat{\psi}_{m_{1}}^{j_{1}} \ldots \widehat{\psi}_{m_{s}}^{j_{s}}
$$

Let now $U$ be an open set trivializing $\lambda \mathcal{E}$; then

$$
\left.y\right|_{U}=\sigma_{G}^{r}\left(R_{U}^{*} \sigma_{G}^{d-1}\left(R_{U}^{*}\right) \ldots \sigma_{G}^{(s-1)(d-1)}\left(R_{U}^{*}\right)\right) \sum_{L M} f_{L M}^{U} \psi_{L} \psi_{L} \widehat{\psi}_{m_{1}}^{U} \ldots \widehat{\psi}_{m_{s}}^{U}
$$

where $R_{U}$ generates locally $\lambda \mathcal{E}, f_{L M}^{U} \in C(U)$ and each $\widehat{\psi}_{m_{k}}^{U}$ is defined by (5.7) replacing $R_{i}$ with $R_{U}$. Thus $\left.y\right|_{U}=T_{U} y_{U}^{\prime}$ where $T_{U}:=\sigma_{G}^{r}(\ldots)$ is an isometry in $\left.\mathcal{F}_{\mu}\right|_{U}$ (as $\left.R_{U}^{*} \in \mathcal{F}_{\mu}\right|_{U}$ and $\mathcal{F}_{\mu}$ is $\sigma_{G}$-stable) and $\left.\left.y\right|_{U} \in\left(\iota, \mathcal{E}^{r+s(d-1)}\right)\right|_{U}$. But $\widehat{g}\left(\left.y\right|_{U}\right)=\widehat{g}\left(T_{U} y_{U}^{\prime}\right)=T_{U} \widehat{g}\left(y_{U}^{\prime}\right)$ and as $\left.y\right|_{U}=\widehat{g}\left(\left.y\right|_{U}\right)$ we find $T_{U} \widehat{g}\left(y_{U}^{\prime}\right)=T_{U} y_{U}^{\prime}$; thus multiplying on the left by $T_{U}^{*}, \widehat{g}\left(y_{U}^{\prime}\right)=y_{U}^{\prime}$. So we conclude that $y_{U}^{\prime} \in$ $\left.\left.\left(\iota, \mathcal{E}^{r+s(d-1)}\right)_{G}\right|_{U} \subset \mathcal{F}_{\mu}\right|_{U}$ and $\left.y\right|_{U}$ belongs to $\left.\mathcal{F}_{\mu}\right|_{U}$. By applying the above argument to each open set of a cover of $\widehat{\mathcal{Z}}^{\rho}$ we get $y \in \mathcal{F}_{\mu}$. 
Thus the $\mathcal{Z}^{\rho}$-module maps (5.45.5) extend to an injective $\mathcal{Z}^{\rho}$-module map of $\mathcal{A} \odot_{\mu}{ }^{0} \mathcal{O}_{\mathcal{E}}$ into end $\left(\mathcal{A} \odot_{\mu}{ }^{0} \mathcal{O}_{\mathcal{E}}\right)$, by posing

$$
\overline{a \otimes_{\mu} t}:=\bar{a} \cdot \bar{t}
$$

in fact by previous lemma $\overline{a \otimes_{\mu} y t}=\overline{a \mu(y) \otimes_{\mu} t}$. Injectivity of (5.12) follows by the equality $\overline{a \otimes_{\mu} t}\left(1 \otimes_{\mu} 1\right)=a \otimes_{\mu} t$. In the same way we obtain that the map

$$
\overline{a \otimes_{\mu} t} \mapsto \overline{a \otimes_{\mu} t}:=\overline{1 \otimes_{\mu} t^{*}} \cdot \overline{a^{*} \otimes_{\mu} 1}
$$

defines an involution on $\mathcal{A} \odot{ }_{\mu}{ }^{0} \mathcal{O}_{\mathcal{E}}$ extending the involutions defined on $\mathcal{A}$, ${ }^{0} \mathcal{O}_{\mathcal{E}}$, as can be proved exactly in the same way of Lemma 2.5 in [19.

In order to economize in notations in the sequel we will identify $\mathcal{A} \odot_{\mu}{ }^{0} \mathcal{O}_{\mathcal{E}}$ with the image in end $\left(\mathcal{A} \odot_{\mu}{ }^{0} \mathcal{O}_{\mathcal{E}}\right)$, so that the overline symbol is dropped. We denote by $i(a):=a \otimes_{\mu} 1, j(t):=1 \otimes_{\mu} t$ the canonical immersions $\mathcal{A},{ }^{0} \mathcal{O}_{\mathcal{E}} \hookrightarrow$ $\mathcal{A} \odot{ }_{\mu}{ }^{0} \mathcal{O}_{\mathcal{E}}$. The following theorem is the solution to our initial problem at the *-algebraic level.

Theorem 5.5. Let $\mathcal{A}$ be a unital $C^{*}$-algebra, $\rho$ a unital endomorphism on $\mathcal{A}$, $\mu$ a dual action of a closed group $G \subseteq \mathbf{S U \mathcal { E }}$ on $(\mathcal{A}, \rho)$, where $\mathcal{E} \rightarrow \widehat{\mathcal{Z}}^{\rho}$ is a vector bundle. Then

- for each commutative diagram of *-algebra morphisms

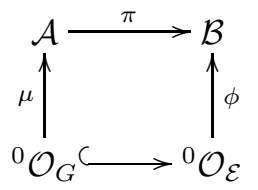

such that $\phi(f)=\pi(f), \phi(\psi) \pi(a)=\pi \circ \rho(a) \phi(\psi)$ there is a unique $\mathcal{Z}^{\rho}$ module ${ }^{*}$-homomorphism $\chi: \mathcal{A} \odot \odot_{\mu}{ }^{0} \mathcal{O}_{\mathcal{E}} \rightarrow \mathcal{B}$ such that $\chi\left(a \otimes_{\mu} t\right)=$ $\pi(a) \phi(t)$;

- There is a natural $G$-action $\alpha$ on $\mathcal{A} \odot_{\mu}{ }^{0} \mathcal{O}_{\mathcal{E}}$ by ${ }^{*}$-automorphisms such that $\alpha_{g}\left(a \otimes_{\mu} t\right)=a \otimes_{\mu} \widehat{g}(t)$.

Proof. We prove first point. By universality the application $\chi$ exists as a $\mathcal{Z}^{\rho}$ module map. Thus we have to prove that $\chi$ is a ${ }^{*}$-homomorphism, and this can be done in the same way of [19], Th. 2.6. About second assertion, note that by Lemma 5.4 the identity $\mu(y)=y$ (i.e. $i \circ \mu(y)=j(y)$ ) hold for each $y$; thus we can construct a commutative diagram as in first point, with $\mathcal{B}=\mathcal{A} \odot_{\mu}{ }^{0} \mathcal{O}_{\mathcal{E}}$, $i=\pi, j \circ \widehat{g}=\phi$. Furthermore $j \circ \widehat{g}(f)=i(f)$ for $f \in \mathcal{Z}^{\rho}$ and

$$
i \circ \widehat{g}(\psi) \cdot i(a)=1 \otimes_{\mu} \widehat{g}(\psi) \cdot a \otimes_{\mu} 1=\rho(a) \otimes_{\mu} 1 \cdot 1 \otimes_{\mu} \widehat{g}(\psi)=i \circ \rho(a) \cdot j \circ \widehat{g}(\psi),
$$


so that we found that property (5.2) holds for elements of $i(\mathcal{A})$ and $j \circ \widehat{g}\left({ }^{0} \mathcal{O}_{\mathcal{E}}\right)$. Thus by universality there is a $\mathcal{Z}^{\rho}$-module ${ }^{*}$-homomorphism $\alpha_{g}: \mathcal{A} \odot_{\mu}{ }^{0} \mathcal{O}_{\mathcal{E}} \rightarrow$ $\mathcal{A} \odot_{\mu}{ }^{0} \mathcal{O}_{\mathcal{E}}$. Now $\alpha_{g}$ has inverse $\alpha_{g^{*}}$ and it is obvious that $\alpha_{g g^{\prime}}=\alpha_{g} \alpha_{g^{\prime}}$.

Our aim is now to extend previous theorem in such a way to get (5.15.2) in the setting of $C^{*}$-algebra morphisms. It is clear that the solution will be given by the closure w.r.t. some suitable $C^{*}$-norm of the ${ }^{*}$-algebra $\mathcal{A} \odot_{\mu}{ }^{0} \mathcal{O}_{\mathcal{E}}$ constructed in first part of the present section. At this purpose we construct a family of $C^{*}$-seminorms for $\mathcal{A} \odot_{\mu}{ }^{0} \mathcal{O}_{\mathcal{E}}$, indexed by elements of the spectrum of $\mathcal{Z}^{\rho}$.

Let $x \in \widehat{\mathcal{Z}}^{\rho}$ and $\operatorname{ker} x$ be the corresponding ideal of $\mathcal{Z}^{\rho}$. We consider the ideal $\mathcal{A} \operatorname{ker} x$, introduce the notation $\mathcal{A}_{x}$ to indicate the quotient of $\mathcal{A}$ by $\mathcal{A}$ ker $x$, and denote by $\pi_{x}: \mathcal{A} \rightarrow \mathcal{A}_{x}$ the natural surjection. Note that by [8], 9] we have $\|a\|=\sup _{x}\left\|\pi_{x}(a)\right\|$ for each $a \in \mathcal{A}$. Let now $G_{x}$ be the fibre of $G$ over $x$. We denote by $x_{*}: \mathcal{O}_{\mathcal{E}} \rightarrow \mathcal{O}_{d}$ the evaluation at $x$ of $\mathcal{O}_{\mathcal{E}}$ as a continuous field of $C^{*}$-algebras. We want to construct a morphism $\mu_{x}: \mathcal{O}_{G_{x}} \rightarrow \mathcal{A}_{x}$ such that $\pi_{x} \circ \mu=\mu_{x} \circ x_{*}$. In the same way we want to define an endomorphism $\rho_{x}: \mathcal{A}_{x} \rightarrow \mathcal{A}_{x}$ such that $\pi_{x} \circ \rho=\rho_{x} \circ \pi_{x}$. In order to define $\mu_{x}$ we will make use of the following lemma:

Lemma 5.6. Let $\left(\mathcal{F}, \mathcal{F} \rightarrow \mathcal{F}_{x}\right)$ be a continuous field of $C^{*}$-algebras over a compact space $X, U \subseteq X$ a closed set. If $y \in \mathcal{F}$ is a vector field such that the restriction $\left.y\right|_{U}=0$ then there exists a continuous function $f \in C(X)$ such that $\left.f\right|_{U}=0$ and a vector field $y^{\prime} \in \mathcal{F}$ with $y=f y^{\prime}$.

Proof. It suffices to regard at the $C^{*}$-algebra $\mathcal{F}_{U}:=\left\{y \in \mathcal{F}:\left.y\right|_{U}=0\right\}$ as a non degenerate Banach $C(X-U)$-bimodule and apply [10] or [9], Prop. 1.8.

We now give the following definitions:

$$
\begin{aligned}
& \mu_{x} \circ x_{*}(y):=\pi_{x} \circ \mu(y) \\
& \rho_{x} \circ \pi_{x}(a):=\pi_{x} \circ \rho(a)
\end{aligned}
$$

As $\mu$ is a $\mathcal{Z}^{\rho}$-module map by the previous lemma it follows that if $y_{x}:=x_{*}(y)=$ 0 then $\pi_{x}(\mu(y))=f(x) \cdot \pi_{x}(\mu(y))=0$, so that $\mu_{x}$ is well defined. For $\rho_{x}$, note that if $\pi_{x}(a)=0$ then $a$ is norm limit of terms of the type $\sum_{k} f_{k} a_{k}$, where $f_{k} \in \operatorname{ker} x \subset \mathcal{Z}^{\rho}, a_{k} \in \mathcal{A}$. Thus $\pi_{x}(\rho(a))=0$ (as the restriction of $\pi_{x}$ to $\mathcal{Z}^{\rho}$ is the evaluation on $x$ ) and $\rho_{x}$ is well defined. We can now prove the following

Proposition 5.7. For each $x \in \widehat{\mathcal{Z}}^{\rho}$, the morphism $\mu_{x}:\left(\mathcal{O}_{G}\right)_{x} \rightarrow \mathcal{A}_{x}$ defines a dual $G_{x}$-action $\widehat{G}_{x} \rightarrow$ end $\mathcal{A}_{x}$ (where $G_{x} \subseteq \mathbb{S U}(d)$ is the closed group defined in Lemma 3.3). 
Proof. We constructed for every $x \in \widehat{\mathcal{Z}}^{\rho}$ an homomorphism $\mu_{x}:\left(\mathcal{O}_{G}\right)_{x} \simeq$ $\mathcal{O}_{G_{x}} \rightarrow \mathcal{A}_{x}$. First of all we have to verify that $\rho_{x} \circ \mu_{x}=\mu_{x} \circ \sigma_{x}$, where $\sigma_{x}$ is the canonical endomorphism of $\mathcal{O}_{G_{x}}$. By definition if $y_{x} \in{ }^{0} \mathcal{O}_{G_{x}}$ we find $\rho_{x} \circ \mu_{x}\left(y_{x}\right)=\rho_{x} \circ \pi_{x} \circ \mu(y)=\pi_{x} \circ \rho \circ \mu(y)=\pi_{x} \circ \mu \circ \sigma(y)=\mu_{x} \circ \pi_{x} \circ \sigma(y)=$ $\mu_{x} \circ \sigma_{x}\left(y_{x}\right)$. We now prove that $\mu_{x}\left(\sigma_{x}^{r}, \sigma_{x}^{s}\right) \subseteq\left(\rho_{x}^{r}, \rho_{x}^{s}\right)$ for every $r, s \in \mathbb{N}$ : if $y_{x} \in\left(\sigma_{x}^{r}, \sigma_{x}^{s}\right)$ then for every $\pi_{x}(a) \in \mathcal{A}_{x}$ we find $\mu_{x}\left(y_{x}\right) \cdot \rho_{x}^{r} \circ \pi_{x}(a)=$ $\pi_{x} \circ \mu(y) \cdot \pi_{x} \circ \rho^{r}(a)=\pi_{x}\left(\mu(y) \cdot \rho^{r}(a)\right)=\pi_{x}\left(\rho^{s}(a) \cdot \mu(y)\right)=\rho_{x}^{s} \circ \pi_{x}(a) \cdot \mu_{x}\left(y_{x}\right)$, and the assert is proved.

We can now apply the results of [19] and define $\mathcal{A}_{x} \otimes_{\mu_{x}} \mathcal{O}_{d}$ as the closure w.r.t. the maximal $C^{*}$-seminorm of the ${ }^{*}$-algebra $\mathcal{A}_{x} \odot_{\mu_{x}}{ }^{0} \mathcal{O}_{d}$, obtained by the dual $G_{x}$-action defined in previous proposition. By [19], Thm. $3.2 \mathcal{A}_{x} \otimes_{\mu_{x}} \mathcal{O}_{d}$ is the solution of our universal problem in the case of 'flat' dual $G_{x}$-action on $\left(\mathcal{A}_{x}, \rho_{x}\right)$.

Lemma 5.8. For each $x \in \widehat{\mathcal{Z}}^{\rho}$ there is a ${ }^{*}$-homomorphism $\widehat{x}: \mathcal{A} \odot_{\mu}{ }^{0} \mathcal{O}_{\mathcal{E}} \rightarrow$ $\mathcal{A}_{x} \otimes_{\mu_{x}} \mathcal{O}_{d}$ extending the evaluation morphisms $\pi_{x}, x_{*}$.

Proof. In order to verify that $\widehat{x}\left(a \otimes_{\mu} t\right):=\pi_{x}(a) \otimes_{\mu_{x}} x_{*}(t)$ is well defined we have to prove that it factorizes in the right way on elements of ${ }^{0} \mathcal{O}_{G}$. But this follows immediately by construction of $\mu_{x}$.

We introduce on $\mathcal{A} \odot{ }_{\mu}{ }^{0} \mathcal{O}_{\mathcal{E}}$ the $C^{*}$-seminorm $\|\cdot\|:=\sup _{x}\|\widehat{x}(\cdot)\|$, and denote by $\mathcal{A} \rtimes_{\mu} \widehat{G}$ the closure of $\mathcal{A} \odot_{\mu}{ }^{0} \mathcal{O}_{\mathcal{E}}$ w.r.t. $\|\cdot\|$.

By construction $\|a\|=\left\|a \otimes_{\mu} 1\right\|$ and $\|t\|=\left\|1 \otimes_{\mu} t\right\|$ for $a \in \mathcal{A}, t \in \mathcal{O}_{\mathcal{E}}$. This implies that there are unital monomorphisms $i: \mathcal{A} \hookrightarrow \mathcal{A} \rtimes_{\mu} \widehat{G}$ and $j$ : $\mathcal{O}_{\mathcal{E}} \hookrightarrow \mathcal{A} \rtimes_{\mu} \widehat{G}$. Note that in general $\mathcal{A} \rtimes_{\mu} \widehat{G}$ is not a continuous field of $C^{*}$ algebras over $\widehat{\mathcal{Z}}^{\rho}$, but simply a $\mathcal{Z}^{\rho}$-algebra in the sense of [27. By general facts on $C(X)$-algebras ([27, [31 [8], 9]) we have a picture of $\mathcal{A}$ and $\mathcal{A} \rtimes_{\mu} \widehat{G}$ in terms of an upper semicontinuous field of $C^{*}$-algebras over $\widehat{\mathcal{Z}}^{\rho}$, with fibre respectively $\mathcal{A}_{x}$ and $\mathcal{A}_{x} \otimes_{\mu_{x}} \mathcal{O}_{d}$.

Theorem 5.9. Let $\rho$ be a unital endomorphism of a $C^{*}$-algebra $\mathcal{A}, G \subseteq \mathbf{S U E}$ a closed group of special unitaries on a vector bundle $\mathcal{E} \rightarrow \widehat{\mathcal{Z}}^{\rho}$ with a dual action $\mu: \widehat{G} \rightarrow \widehat{\rho} \subset$ end $\mathcal{A}$. Then $\mathcal{A} \rtimes_{\mu} \widehat{G}$ is the unique crossed product of $\mathcal{A}$ by $\mu$ satisfying universal properties $5.1,5.2)$. $\mathcal{A} \rtimes_{\mu} \widehat{G}$ carries a strongly continuous $G$-action $\alpha$, with $\alpha_{g}\left(a \otimes_{\mu} t\right)=a \otimes_{\mu} \widehat{g}(t)$. The fixed point algebra for $\alpha$ is $\mathcal{A} \otimes_{\mu} 1 \simeq \mathcal{A}$.

Proof. First we prove unicity of the $C^{*}$-norm. Let $\mathcal{B}$ be the completition of $\mathcal{A} \odot_{\mu}{ }^{0} \mathcal{O}_{\mathcal{E}}$ w.r.t. a given $C^{*}$-norm; note that $\mathcal{B}$ is a $\mathcal{Z}^{\rho}$-algebra. We require that the above defined action $\alpha$ has to be isometric. As each $\alpha_{g}$ is a $\mathcal{Z}^{\rho}$-morphism it defines a family of automorphisms $\alpha_{x} \in \mathcal{B}_{x}$, where $\mathcal{B}_{x}$ is the fibre of $\mathcal{B}$ on $x \in X$ as an upper semicontinuous field. By [19], Thm. 3.2 the unique norm 
such that $\alpha_{x}$ is isometric is the maximal $C^{*}$-norm, so that $\mathcal{B}_{x}=\mathcal{A}_{x} \otimes_{\mu_{x}} \mathcal{O}_{d}$. Thus $\mathcal{B}=\mathcal{A} \rtimes_{\mu} \widehat{G}$. We now prove that the fixed point algebra w.r.t. $G$-action is $\mathcal{A}$. At this purpose note that if $b \in \mathcal{A} \rtimes_{\mu} \widehat{G}$ is $G$-invariant then for every $x \in \widehat{\mathcal{Z}}^{\rho}$ we find that $b_{x} \in \mathcal{A}_{x} \otimes_{\mu_{x}} \mathcal{O}_{d}$ is $G_{x}$-invariant. By using invariant $G_{x}$-invariant $\mathcal{A}_{x}$-valued mean on $\mathcal{A}_{x} \otimes_{\mu_{x}} \mathcal{O}_{d}$ (as in [19]) we deduce that $b_{x} \in \mathcal{A}_{x}$. Thus $b$ defines a vector field belonging to the upper semicontinuous bundle spanned by $\mathcal{A}$, i.e. $b$ belongs to $\mathcal{A}$.

Note that when $G$ is locally trivial, in order to prove that $\left(\mathcal{A} \rtimes_{\mu} \widehat{G}\right)^{\alpha}=\mathcal{A}$ we can directly apply an invariant mean argument by using the $C(X)$-valued measure defined in 42, without passing to study behaviour along the fibres. Universality implies the following corollary, establishing a funtoriality property w.r.t. the group $G \subseteq \mathbf{S U E}$.

Corollary 5.10. Let $G \subseteq \mathbf{S U E}$ be a closed group with a dual action $\mu: \widehat{G} \rightarrow$ $\widehat{\rho}$. Suppose $G^{\prime} \subseteq \mathbf{S U E}$ is a closed group with $G \subseteq G^{\prime}$. Then a dual $G^{\prime}$ action $\mu^{\prime}$ is defined on $(\mathcal{A}, \rho)$, and there is a surjective $C^{*}$-algebra morphism $\eta: \mathcal{A} \rtimes_{\mu^{\prime}} \widehat{G^{\prime}} \rightarrow \mathcal{A} \rtimes_{\mu} \widehat{G}$. The morphism $\eta$ preserves $\mathcal{A}$, and intertwines actions by $G, G^{\prime}$ as well as inner endomorphisms induced by $\widehat{\mathcal{E}}$ with their intertwiners spaces $\left(\sigma_{\mathcal{E}}^{r}, \sigma_{\mathcal{E}}^{s}\right)$.

Proof. It is clear that $\widehat{G^{\prime}} \subseteq \widehat{G}$, so that $\mu$ restricts to a dual $G^{\prime}$-action $\mu^{\prime}$ on $(\mathcal{A}, \rho)$. As $\mathcal{O}_{G^{\prime}} \subseteq \mathcal{O}_{G}$, the crossed product $\mathcal{A} \rtimes_{\mu} \widehat{G}$ satisfies universal properties (5.15.2) for the dual $G^{\prime}$-action $\mu^{\prime}$, so that there must exists the required morphism $\eta$ extending the natural map $\mathcal{A} \odot{ }_{\mu^{\prime}}{ }^{0} \mathcal{O}_{\mathcal{E}} \rightarrow \mathcal{A} \odot_{\mu}{ }^{0} \mathcal{O}_{\mathcal{E}}$.

Of particular interest for our purposes will be crossed products by dual actions with minimal relative commutant of $\mathcal{A}$. We denote by $\operatorname{aut}_{\mathcal{A}}\left(\mathcal{A} \rtimes_{\mu} \widehat{G}, \sigma_{\mathcal{E}}\right)$ the group of automorphisms on $\mathcal{A} \rtimes_{\mu} \widehat{G}$ leaving $\mathcal{A}$ pointwise fixed and commuting with $\sigma_{\mathcal{E}}$. As usual the notation $S(X, \mathcal{G})$ denotes the group of continuous sections of the spectral bundle $\mathcal{G} \rightarrow X$ spanned by $G$.

Proposition 5.11. Let $\rho$ be a unital endomorphism on a $C^{*}$-algebra $\mathcal{A}, G$ a closed group of special unitaries on a vector bundle $\mathcal{E} \rightarrow \widehat{\mathcal{Z}}^{\rho}$ with a dual action $\mu: \widehat{G} \rightarrow \widehat{\rho} \subset$ end $\mathcal{A}$. Suppose $\mathcal{A}^{\prime} \cap\left(\mathcal{A} \rtimes_{\mu} \widehat{G}\right)=\mathcal{Z}$. Then the Banach space

$$
\mathcal{M}:=\left\{\varphi \in \mathcal{A} \rtimes_{\mu} \widehat{G}: \varphi a=\rho(a) \varphi, a \in \mathcal{A}\right\}
$$

is a Hilbert $\mathcal{Z}$-bimodule in $\mathcal{A} \rtimes_{\mu} \widehat{G}$, and a noncommutative pullback of $\mathcal{E}$. Furthermore $G$ acts on $\mathcal{M}$ by unitary $\mathcal{Z}$-bimodule maps, and $\mathcal{O}_{\mathcal{M}}^{G} \subset \mathcal{B}_{\mathcal{M}}$. Finally, $S(X, \mathcal{G}) \simeq \operatorname{aut}_{\mathcal{A}}\left(\mathcal{A} \rtimes_{\mu} \widehat{G}, \sigma_{\mathcal{E}}\right)$.

Proof. It is clear that $\widehat{\mathcal{E}} \cdot \mathcal{Z} \subseteq \mathcal{M}$; viceversa, $\psi^{*} \varphi \in \mathcal{A}^{\prime} \cap\left(\mathcal{A} \rtimes_{\mu} \widehat{G}\right)=\mathcal{Z}$ for $\psi \in \widehat{\mathcal{E}}, \varphi \in \mathcal{M}$, and $\varphi f=f \varphi$ for $\varphi \in \mathcal{M}, f \in \mathcal{Z}^{\rho}$. Furthermore, note that $z=\alpha_{g}(z)$ for $z \in \mathcal{Z}, g \in G$. Thus $\alpha_{g}(z \varphi)=z \alpha_{g}(\varphi), \alpha_{g}(\varphi z)=\alpha_{g}(\varphi) z$, and $\alpha_{g}\left(\varphi^{*} \varphi^{\prime}\right)=\varphi^{*} \varphi^{\prime}$ for $\varphi, \varphi^{\prime} \in \mathcal{M}$ (in fact $\left.\varphi^{*} \varphi^{\prime} \in \mathcal{Z}\right), g \in G$, and $G$ acts 
unitarely on $\mathcal{M}$ by $\mathcal{Z}$-bimodule maps. Furthermore, by construction $\mathcal{O}_{\mathcal{M}}^{G} \subseteq$ $\mathcal{A} \subseteq \mathcal{Z}^{\prime} \cap\left(\mathcal{A} \rtimes_{\mu} \widehat{G}\right)$, thus $\mathcal{O}_{\mathcal{M}}^{G} \subseteq \mathcal{B}_{\mathcal{M}}$. Finally, if $\alpha \in \operatorname{aut}_{\mathcal{A}}\left(\mathcal{A} \rtimes_{\mu} \widehat{G}, \sigma_{\mathcal{E}}\right)$, with the same methods used in Prop. 4.8 (with $\mathcal{A}$ playing the role of $\mathcal{O}_{\mathcal{M}}^{G}$ ) we find that $\alpha=\widehat{g}$ for some $g \in S(X, \mathcal{G})$.

We now give an algebraic characterization of crossed products by dual actions. Let $\mathcal{B}$ be a unital $C^{*}$-algebra acted upon by a topological group $G$. We introduce the notations $\mathcal{A}:=\mathcal{B}^{G}, \mathcal{Z}:=\mathcal{A} \cap \mathcal{A}^{\prime}$. Let us now suppose that there exists a finitely generated $G$-stable Hilbert $\left(\mathcal{B} \cap \mathcal{B}^{\prime}\right)^{G}$-bimodule $\widehat{\mathcal{E}} \subset \mathcal{B}$ with support 1 such that $\mathcal{B}$ is generated by $\mathcal{A}, \widehat{\mathcal{E}}$ as a $C^{*}$-algebra. By Serre-Swan theorem, $\widehat{\mathcal{E}}$ is the module of continuous sections of a vector bundle $\mathcal{E}$. We then use the notation $(\mathcal{B}, G, \mathcal{A}, \mathcal{E})$ to denote such a system. Note that there is an inclusion $\mathcal{O}_{\mathcal{E}} \hookrightarrow \mathcal{B}$, where $\mathcal{O}_{\mathcal{E}}$ is the Pimsner algebra of $\mathcal{E}$. Furthermore $\widehat{\mathcal{E}}$ induces an inner endomorphism $\sigma_{\mathcal{E}}$ on $\mathcal{B}$, and since $\sigma_{\mathcal{E}}$ does not depend on the choice of the generators $\mathcal{A}$ is $\sigma_{\mathcal{E}}$-stable (in fact if $\left\{\psi_{l}\right\}$ is a set of generators of $\widehat{\mathcal{E}}$ the same is true for $\left\{g \psi_{l}\right\}$ for $g \in G$, and $\left.g \circ \sigma_{\mathcal{E}}(b)=\sigma_{\mathcal{E}} \circ g(b)\right)$. We denote by $\rho \in \mathbf{e n d} \mathcal{A}$ the restriction of $\sigma_{\mathcal{E}}$ on $\mathcal{A}$.

Lemma 5.12. Let $(\mathcal{B}, G, \mathcal{A}, \mathcal{E})$ as above. Then the following properties hold:

- $\rho$ has weak permutation symmetry;

- $\mathcal{Z}^{\rho}=\left(\mathcal{B} \cap \mathcal{B}^{\prime}\right)^{G}=\left(\mathcal{B} \cap \mathcal{B}^{\prime}\right) \cap \mathcal{Z}$;

- there is a dual action $\mu: \widehat{G} \rightarrow \widehat{\rho}$, and $\mathcal{B} \simeq \mathcal{A} \rtimes_{\mu} \widehat{G}$.

Proof.

- It suffice to pick a (finite) set of generators $\left\{\psi_{l}\right\}$ of $\widehat{\mathcal{E}}$ and define the map

$$
\varepsilon(p):=\sum_{L} \psi_{l_{p(1)}} \cdots \psi_{l_{p(d)}} \psi_{l_{d}}^{*} \cdots \psi_{l_{1}}^{*},
$$

where $p \in \mathbb{P}_{d}$. As left $\left(\mathcal{B} \cap \mathcal{B}^{\prime}\right)^{G}$-action on $\widehat{\mathcal{E}}$ coincides with the right one (in fact it is simply given by left and right multiplication) the above defined map does not depend on the choice of the generators. For the same reason $\varepsilon(p)$ is $G$-invariant and belongs to $\mathcal{A}$. We leave to the reader the easy verifications of the required properties of weak permutation symmetry.

- It is clear that $\left(\mathcal{B} \cap \mathcal{B}^{\prime}\right)^{G}=\left(\mathcal{B} \cap \mathcal{B}^{\prime}\right) \cap \mathcal{Z}$. Furthermore $\mathcal{Z}^{\rho}$ as defined in (2.1) is contained in $\mathcal{Z} \subset \mathcal{A}=\mathcal{B}^{G}$, and elements of $\mathcal{Z}^{\rho}$ commutes with elements of $\widehat{\mathcal{E}}$ (as $\mathcal{Z}^{\rho}$ is pointwise $\sigma_{\mathcal{E}}$-invariant). Thus $\mathcal{Z}^{\rho} \subseteq\left(\mathcal{B} \cap \mathcal{B}^{\prime}\right) \cap \mathcal{Z}$. Viceversa, it is clear that $\left(\mathcal{B} \cap \mathcal{B}^{\prime}\right) \cap \mathcal{Z}$ is contained in $\mathcal{Z}^{\rho}$.

- We denote by $j: \mathcal{O}_{\mathcal{E}} \hookrightarrow \mathcal{B}$ the natural inclusion. The $G$-action on $\mathcal{B}$ restricts to the natural $G$-action on $\mathcal{O}_{\mathcal{E}}$, regarding at $G$ as a subgroup of $\mathbf{U} \mathcal{E}$. Thus we just have to prove that $j\left(\mathcal{E}^{r}, \mathcal{E}^{s}\right)_{G} \subseteq\left(\rho^{r}, \rho^{s}\right)$. Now 
if $t \in\left(\mathcal{E}^{r}, \mathcal{E}^{s}\right)$ then $t=\sum_{L M} \psi_{L} \psi_{M}^{*} t_{L M}$, where $\left\{\psi_{L}\right\}$ generate $\widehat{\mathcal{E}}^{s}$, $\left\{\psi_{M}\right\}$ generate $\widehat{\mathcal{E}}^{r}, t_{L M} \in \mathcal{Z}^{\rho}$. Furthermore by definition of $G$-action $j(t)$ belongs to $\mathcal{A}$. Thus, as $\sigma^{r}(b) j\left(\psi_{L}\right)=j\left(\psi_{L}\right) b$ for $b \in \mathcal{B}$, we find $\rho^{s}(a) j(t)=\sigma_{\mathcal{E}}^{s}(a) j(t)=j(t) \sigma_{\mathcal{E}}^{r}(a)=j(t) \rho^{r}(a)$ for $a \in \mathcal{A}$. So that the required functor is defined as $\mu\left(\widehat{\mathcal{E}}^{r}\right):=\rho^{r}$ on objects and by the inclusion map $\mu:=\left.j\right|_{\mathcal{O}_{G}}:\left(\mathcal{E}^{r}, \mathcal{E}^{s}\right)_{G} \hookrightarrow\left(\rho^{r}, \rho^{s}\right)$ on arrows. Since the tensor product $t \otimes t^{\prime}=t \otimes 1 \cdot 1 \otimes t^{\prime}$ is translated as $t \sigma_{\mathcal{E}}^{r}\left(t^{\prime}\right)$ in $\mathcal{O}_{\mathcal{E}}$, the functor $\mu$ mantains the tensor structure (in fact $\left.\sigma_{\mathcal{E}}\right|_{\mathcal{A}}=\rho$ ). In such a way we constructed a commutative diagram as in the definition of crossed product by a dual action. As $\mathcal{B}$ is generated by $\mathcal{A}, \widehat{\mathcal{E}}$, and $G$ acts on $\mathcal{B}$ by (isometric) automorphisms, by universality we obtain the desired isomorphism.

Example 5.3. We discuss the special case $(\mathcal{B}, \mathbb{T}, \mathcal{A}, \mathcal{L})$, where $\mathcal{L} \rightarrow X$ is a line bundle with $C(X):=\left(\mathcal{B} \cap \mathcal{B}^{\prime}\right)^{\mathbb{T}}$. By construction $\mathcal{L}$ carries the natural action by $\mathbb{T} \subset \mathcal{U L}=C(X, \mathbb{T})$. Let $\left\{\psi_{l}\right\}$ be a finite set of generators of $\widehat{\mathcal{L}}$. Being $\mathcal{O}_{\mathcal{L}}$ an abelian $C^{*}$-algebra (see [40]) we find that $\sigma_{\mathcal{L}}(\cdot):=\sum_{l} \psi_{l} \cdot \psi_{l}^{*}$ is an automorphism of $\mathcal{B}$ with inverse $\phi_{\mathcal{L}}:=\sum_{l} \psi_{l}^{*} \cdot \psi_{l}$ (in fact $\psi_{l} \psi_{m}^{*}=\psi_{m}^{*} \psi_{l} \in C(X)$ so that $\phi_{\mathcal{L}}$ is multiplicative). For the same reason (5.16) implies that $\varepsilon(p)=1 \forall p \in \mathbb{P}_{\infty}$, and permutation symmetry of $\rho:=\left.\sigma_{\mathcal{L}}\right|_{\mathcal{A}}$ is trivial. We recall (see [40]) that $\left(\mathcal{L}^{r}, \mathcal{L}^{s}\right)=\left(\iota, \mathcal{L}^{r-s}\right)$ for $r>s,\left(\mathcal{L}^{r}, \mathcal{L}^{s}\right)=\left(\mathcal{L}^{s-r}, \iota\right)$ for $r<s,\left(\mathcal{L}^{r}, \mathcal{L}^{r}\right)=C(X)$ for every $r, s \in \mathbb{N}$; so that we find that the category $\widehat{\mathbb{T}}$ has objects the tensor powers $\mathcal{L}^{r}$, with arrows $\left(\mathcal{L}^{r}, \mathcal{L}^{s}\right)_{\mathbb{T}}=\delta_{r, s} \cdot C(X)$, where $\delta_{r, s}$ is the Kroneker

symbol. Thus (being a priori $\left.C(X) \subseteq\left(\rho^{r}, \rho^{s}\right)\right)$ the functor $\mu: \widehat{\mathbb{T}} \rightarrow \operatorname{end} \mathcal{A}$ as defined in previous lemma is completely determined by just assigning the map $\mathcal{L}^{r} \mapsto \rho^{r}, r \in \mathbb{N}$. We can naturally extend to negative integers, by assigning $\mathcal{L}^{-r}:=\left(\mathcal{L}^{*}\right)^{r} \mapsto \rho^{-r}$, where $\mathcal{L}^{*}$ is the dual bundle of $\mathcal{L}$. Thus $\mu$ is actually equivalent to an action by automorphisms $\mathbb{Z} \rightarrow$ aut $\mathcal{A}$.

\section{Special Endomorphisms.}

We start the present section by giving some basic properties about endomorphisms carrying a permutation symmetry, generalizing the analogues in [16. For our purposes will be convenient to start by the notion of weak permutation symmetry (Def 1.1) for an endomorphism $\rho$ of a unital $C^{*}$-algebra $\mathcal{A}$. In fact in the nontrivial centre case property (1.4) is too restrictive for our purposes, implying for $z \in \mathcal{Z}:=\mathcal{A} \cap \mathcal{A}^{\prime}$ that $\rho(z)=z$.

Definition 6.1. Let $\rho$ be a unital endomorphism on a $C^{*}$-algebra $\mathcal{A}$ carrying a weak permutation symmetry $p \mapsto \varepsilon(p), p \in \mathbb{P}_{\infty}$. Are said to be symmetry intertwiners the elements of $\left(\rho^{r}, \rho^{s}\right)$ for which 1.4 holds:

$$
\left[\rho^{r}, \rho^{s}\right]:=\left\{t \in\left(\rho^{r}, \rho^{s}\right): \varepsilon(s, 1) t=\rho(t) \varepsilon(r, 1)\right\}
$$


We denote by $[\rho]$ the $C^{*}$-subcategory of $\widehat{\rho}$ with objects the tensor powers $\rho^{r}$ and arrows $\left[\rho^{r}, \rho^{s}\right]$.

Lemma 6.2. In the setting of the previous definition, the set of symmetry intertwiners is closed for multiplication and $\rho$-stable. So that $[\rho]$ is a symmetric strict tensor $C^{*}$-category.

Proof. First we prove that if $t \in\left[\rho^{r}, \rho^{s}\right], t^{\prime} \in\left[\rho^{s}, \rho^{h}\right]$ then $t^{\prime} t \in\left[\rho^{r}, \rho^{h}\right]$. This fact is obvious by (1.4), that implies the equality

$$
\rho\left(t^{\prime} t\right)=\varepsilon(h, 1) t^{\prime} \varepsilon(1, s) \varepsilon(s, 1) t \varepsilon(1, r) .
$$

We now prove that for each $k \in \mathbb{N}$ the inclusion $\left[\rho^{r}, \rho^{s}\right] \subset\left[\rho^{r+k}, \rho^{s+k}\right]$ holds. At this purpose note that $(1, s+1) \varepsilon(s, 1)=\mathbb{S}^{s}(1,1)$, so $\varepsilon(1, s+1) \varepsilon(s, 1)=\rho^{s}(\varepsilon)$ and $\varepsilon(s+1,1) \rho^{s}(\varepsilon) t=\rho(t) \varepsilon(r+1,1) \rho^{r}(\varepsilon)$ for $t \in\left[\rho^{r}, \rho^{s}\right]$. As $\rho^{s}(\varepsilon) t=t \rho^{r}(\varepsilon)$ and $\rho^{r}\left(\varepsilon^{2}\right)=1$ we find that $t \in\left[\rho^{r+1}, \rho^{s+1}\right]$. The facts proved above imply that the set of symmetry intertwiners is closed for multiplication. In order to prove that $[\rho]$ is $\rho$-stable it suffice now to verify that $\varepsilon(r, 1) \in\left[\rho^{r+1}, \rho^{r+1}\right]$. But this fact is obviously true, as the relation $(r+1,1)(r, 1)(1, r+1)=\mathbb{S}(r, 1)$ implies that $\varepsilon(r, 1)$ satisfies (1.4). Symmetry follows by the fact that (1.4) implies $t \rho^{r^{\prime}}\left(t^{\prime}\right)=\varepsilon\left(r^{\prime}, s^{\prime}\right) t^{\prime} \rho^{r}(t) \varepsilon(r, s)$ for $t \in\left[\rho^{r}, \rho^{s}\right], t \in\left[\rho^{r^{\prime}}, \rho^{s^{\prime}}\right]$.

We denote by $\mathcal{O}_{[\rho]}$ the $C^{*}$-algebra generated by the $\left[\rho^{r}, \rho^{s}\right]$ 's. By previous lemma follows that $\mathcal{O}_{[\rho]}$ is $\rho$-stable. Note that $[\iota, \iota]=\mathcal{Z}^{\rho}$, so that $\mathcal{O}_{[\rho]}$ is a $\mathcal{Z}^{\rho}$-algebra. From a categorical point of view, the strict tensor $C^{*}$-category $[\rho]$ actually exhibit the symmetry property of tensor product inherited by $\widehat{\rho}$. Anyway in general $[\rho]$ is not a DR-category, since may don't have conjugates in the sense of Doplicher-Roberts, as well may carry nontrivial intertwiners of the identity automorphism (in the particular case considered in [6], $[\rho]$ is actually a DR-category, denoted there by $\left.\mathcal{T}_{\mathbb{C}}\right)$. Symmetry intertwiners naturally appear when there is a dual action $\mu$ of some $G \subset \mathbf{U E}$ on $(\mathcal{A}, \rho)$. In this case $\rho$ has weak permutation symmetry and $\mu\left(\sigma_{G}^{r}, \sigma_{G}^{s}\right) \subset\left[\rho^{r}, \rho^{s}\right]$ for each $r, s$. In fact the canonical endomorphism $\sigma_{G} \in \mathbf{e n d} \mathcal{O}_{G}$ has permutation symmetry (see Sec. 3), and with $\varepsilon(p):=\mu \circ \theta(p), p \in \mathbb{P}_{\infty}$ we find $\varepsilon(s, 1) \cdot \mu(y)=\mu \circ \sigma_{G}(y) \cdot \varepsilon(r, 1)=$ $\rho \circ \mu(y) \cdot \varepsilon(r, 1)$ for $y \in\left(\sigma_{G}^{r}, \sigma_{G}^{s}\right)$. In particular, every endomorphism carrying weak permutation symmetry has symmetry intertwiners thanks to the naturally induced dual $C\left(\widehat{\mathcal{Z}}^{\rho}, \mathbb{U}(d)\right)$-action (see Lemma 6.8 in present section).

We can now give the notion of permutation symmetry in our general setting, naturally coinciding with Def 1.1 when formulated in the trivial centre case. In the general case above remarks imply that Def 1.1 is more restrictive w.r.t. the following

Definition 6.3 (Permutation symmetry). A unital endomorphism $\rho$ of a $C^{*}$-algebra $\mathcal{A}$ with centre $\mathcal{Z}$ has permutation symmetry if there is a unitary representation $p \mapsto \varepsilon(p)$ of the group $\mathbb{P}_{\infty}$ of finite permutations of $\mathbb{N}$ in $\mathcal{A}$ 
such that:

$$
\begin{gathered}
\varepsilon(\mathbb{S} p)=\rho \circ \varepsilon(p) \\
\varepsilon:=\varepsilon(1,1) \in\left(\rho^{2}, \rho^{2}\right) \\
\left(\rho^{r}, \rho^{s}\right)=\rho^{s}(\mathcal{Z}) \cdot\left[\rho^{r}, \rho^{s}\right]=\left[\rho^{r}, \rho^{s}\right] \cdot \rho^{r}(\mathcal{Z})
\end{gathered}
$$

Thus notion of weak permutation symmetry is not affected by Def 6.3 and remains the one given in Def 1.1 Property (6.3) is to be intended in the sense that $\left(\rho^{r}, \rho^{s}\right)$ is spanned by elements of the type $\rho^{s}(\mathcal{Z}) \cdot\left[\rho^{r}, \rho^{s}\right]=\left[\rho^{r}, \rho^{s}\right] \cdot \rho^{r}(\mathcal{Z})$.

Of course above defined permutation symmetry is satisfied by the canonical endomorphisms $\sigma_{G}$ of $C^{*}$-algebras $\mathcal{O}_{G}$, where $G$ is a closed group of unitaries on a vector bundle $\mathcal{E} \rightarrow X$, with $\left[\sigma_{G}^{r}, \sigma_{G}^{s}\right]=\left(\sigma_{G}^{r}, \sigma_{G}^{s}\right)$. Next result supplies a more general class of examples for previously defined notion of permutation symmetry. We refere the reader to the notations introduced in Sec 4.

Proposition 6.4. Let $\mathcal{M} \simeq \widehat{\mathcal{E}} \otimes_{C(X)} C\left(X^{\prime}\right)$ be a noncommutative pullback, $G \subseteq \mathbf{U E}$ a closed group acting on $\mathcal{M}$ by bimodule maps (i.e. $G \subseteq \mathcal{B U} \mathcal{M}$ ) such that $\mathcal{O}_{\mathcal{M}}^{G} \subseteq \mathcal{B}_{\mathcal{M}}$. Then permutation symmetry Def 6.3 is satisfied by the canonical endomorphism $\tau_{G}$ of $\mathcal{O}_{\mathcal{M}}^{G}$.

Proof. The endomorphism $\tau_{G}$ naturally carries weak permutation symmetry $\theta \mapsto \theta(p), p \in \mathbb{P}_{\infty}$ induced by the inner endomorphism $\sigma_{\mathcal{E}}$ of $\mathcal{O}_{\mathcal{M}}$ (see Sec 4 ). By Prop. 4.8 we just have to prove that $\left(\mathcal{E}^{r}, \mathcal{E}^{s}\right)_{G}=\left[\tau_{G}^{r}, \tau_{G}^{s}\right]$. Now $\left(\mathcal{E}^{r}, \mathcal{E}^{s}\right)_{G}=$ $\left(\sigma_{G}^{r}, \sigma_{G}^{s}\right)$ (see Sec. B), so that $\left(\mathcal{E}^{r}, \mathcal{E}^{s}\right)_{G} \subseteq\left[\tau_{G}^{r}, \tau_{G}^{s}\right]$. Viceversa if $t \in\left[\tau_{G}^{r}, \tau_{G}^{s}\right]$ then $t=\sum_{L M} \psi_{L} t_{L M} \psi_{M}^{*}$, where $\left\{\psi_{l}\right\}$ is a finite set of generators of $\widehat{\mathcal{E}}, \psi_{L}:=$ $\psi_{l_{1}} \cdots \psi_{l_{s}} \in\left(\iota, \mathcal{E}^{s}\right) \simeq \widehat{\mathcal{E}}^{s}, \psi_{M} \in\left(\iota, \mathcal{E}^{r}\right), t_{L M}:=\psi_{L}^{*} t \psi_{M} \in[\iota, \iota]$. Thus $t \in$ $\left(\mathcal{E}^{r}, \mathcal{E}^{s}\right)$ and $\left[\tau_{G}^{r}, \tau_{G}^{s}\right] \subseteq\left(\mathcal{E}^{r}, \mathcal{E}^{s}\right)_{G}$.

The role of $\widehat{\rho}$ and $[\rho]$ can be clarified in terms of group duality in the case of crossed products by dual actions with minimal relative commutant of $\mathcal{A}$ (see Prop. 5.11). In that case the noncommutative pullback (5.15) is acted upon by $G$ and the $C^{*}$-category $\widehat{G}_{\mathcal{M}}$ defined in Def. 4.7 is a strict tensor $C^{*}$-category (since $\mathcal{O}_{\mathcal{M}}^{G} \subseteq \mathcal{B}_{\mathcal{M}}$ by Prop. 5.11 and $\widehat{G}_{\mathcal{M}}$ is contained in the strict tensor $C^{*}$-category of tensor powers of $\mathcal{M}$ with arrows bimodule maps).

Lemma 6.5. Let $\mathcal{A} \rtimes_{\mu} \widehat{G}$ be the crossed product by a dual action $\mu: \widehat{G} \rightarrow \widehat{\rho} \subset$ end $\mathcal{A}$ with $\mathcal{A}^{\prime} \cap\left(\mathcal{A} \rtimes_{\mu} \widehat{G}\right)=\mathcal{Z}$. Then $\rho$ has permutation symmetry Def 6.3, and there are isomorphisms of strict tensor $C^{*}$-categories $\widehat{G} \simeq[\rho]$ and $\widehat{G}_{\mathcal{M}} \simeq \widehat{\rho}$, where $\mathcal{M}$ is the noncommutative pullback defined by (5.15). 
Proof. Spaces of arrows of $\widehat{G}_{\mathcal{M}}$ appear in $\mathcal{B}:=\mathcal{A} \rtimes_{\mu} \widehat{G}$ as $\left(\mathcal{M}^{r}, \mathcal{M}^{s}\right)_{G} \simeq$ $\left(\mathcal{M}^{s} \cdot \mathcal{M}^{r *}\right) \cap \mathcal{A}$. We denote by $j: \mathcal{O}_{\mathcal{M}} \hookrightarrow \mathcal{B}$ the natural inclusion. Thus if $t \in\left(\mathcal{M}^{r}, \mathcal{M}^{s}\right)_{G}$ then $t=\sum_{L M} \psi_{L} t_{L M} \psi_{M}^{*}$, where $\psi_{L} \in \widehat{\mathcal{E}}^{s}, \psi_{M} \in \widehat{\mathcal{E}}^{r}, t_{L M} \in$ $\mathcal{Z}$. Now, $j\left(\mathcal{M}^{r}, \mathcal{M}^{s}\right)_{G} \subset \mathcal{A}$, and $j(t) \rho^{r}(a)=\rho^{s}(a) j(t)$ for every $a \in \mathcal{A}$, as is verified by using above expression for $t$. Thus we verified that $j\left(\mathcal{M}^{r}, \mathcal{M}^{s}\right)_{G} \subseteq$ $\left(\rho^{r}, \rho^{s}\right)$. Viceversa, if $t^{\prime} \in\left(\rho^{r}, \rho^{s}\right)$ then $t_{L M}^{\prime}:=\psi_{L}^{*} t^{\prime} \psi_{M} \in \mathcal{A}^{\prime} \cap \mathcal{B}=\mathcal{Z}$ and $t^{\prime} \in\left(\mathcal{M}^{s} \cdot \mathcal{M}^{r *}\right)$. Thus $t^{\prime} \in\left(\mathcal{M}^{s} \cdot \mathcal{M}^{r *}\right) \cap \mathcal{A}=j\left(\mathcal{M}^{r}, \mathcal{M}^{s}\right)_{G}$. We proved in this way also that $\left(\mathcal{O}_{\mathcal{M}}^{G}, \tau_{G}\right) \simeq\left(\mathcal{O}_{\rho}, \rho\right)$, where $\tau_{G}$ denotes the canonical shift endomorphism on $\mathcal{O}_{\mathcal{M}}^{G}$, since by definition of crossed product by dual action $j \circ \tau_{G}=\sigma_{\mathcal{E}} \circ j$. We now prove that $j$ restricts to an isomorphism $\mathcal{O}_{G} \rightarrow \mathcal{O}_{[\rho]}$. It is clear that $j\left(\mathcal{E}^{r}, \mathcal{E}^{s}\right)_{G} \subseteq\left[\rho^{r}, \rho^{s}\right]$; thus we have just to verify that $\left[\rho^{r}, \rho^{s}\right] \subseteq j\left(\mathcal{E}^{r}, \mathcal{E}^{s}\right)_{G}$ for each $r, s \in \mathbb{N}$. At this purpose it suffice to observe that if $t \in\left[\rho^{r}, \rho^{s}\right]$ then with the above notations we find $\rho\left(t_{L M}\right)=$ $\sigma_{\mathcal{E}}\left(\psi_{L}^{*}\right) \rho(t) \sigma_{\mathcal{E}}\left(\psi_{M}\right)=\sigma_{\mathcal{E}}\left(\psi_{L}^{*}\right) \varepsilon(s, 1) t \varepsilon(1, r) \sigma_{\mathcal{E}}\left(\psi_{M}\right)=t_{L M}$ (in fact $\varepsilon(s, 1) \psi_{L}=$ $\left.\sigma_{\mathcal{E}}\left(\psi_{L}\right)\right)$. Thus $t_{L M} \in \mathcal{Z}^{\rho}, t \in j\left(\mathcal{O}_{\mathcal{E}}\right)$ and the inclusion is proved. Now $\rho$ carries weak permutation symmetry by Lemma 5.12 furthermore permutation symmetry holds by previously established isomorphism $\widehat{\rho} \simeq \widehat{G}_{\mathcal{M}}$ and Prop. 6.4

Example 6.1. Dynamical $C^{*}$-systems of the type $\left(\mathcal{B}, \mathbb{T}, \mathcal{A}, S^{1} \times \mathbb{C}\right)$ with notations of Lemma 5.12 studied in 4 . Such systems present a structure of crossed product by dual action $\mathcal{B} \simeq \mathcal{A} \rtimes_{\mu} \widehat{\mathbb{T}}$ with minimality condition $\mathcal{A}^{\prime} \cap \mathcal{B}=\mathcal{A} \cap \mathcal{A}^{\prime}$; note that by $\operatorname{Ex}[5.3$ the dual action $\mu: \widehat{\mathbb{T}} \rightarrow$ end $\mathcal{A}$ is actually an action by automorphisms $\mathbb{Z} \rightarrow$ aut $\mathcal{A}$.

Example 6.2. Let $\mathcal{E} \rightarrow X$ be a vector bundle over a compact Hausdorff space $X, G \subseteq \mathbf{U E}$ a closed group. Then $\mathcal{O}_{\mathcal{E}}=\mathcal{O}_{G} \rtimes_{\mu} \widehat{G}$ (see Ex [5.2) and by Prop. 3.1 minimality condition is satisfied.

Further examples of such crossed products by dual actions with minimal relative commutant of $\mathcal{A}$ are given by Prop. 4.8 (i.e. group actions on noncommutative pullbacks). We will return in next section on such $C^{*}$-systems.

We now establish some basic properties of endomorphisms carrying (weak) permutation symmetry.

Lemma 6.6. Let $\mathcal{A}$ be a unital $C^{*}$-algebra, $\mathcal{Z}_{0}$ a unital $C^{*}$-subalgebra of the centre of $\mathcal{A}, \mu_{0}: \mathcal{Z}_{0} \otimes \mathcal{O}_{\mathbb{U}(d)} \rightarrow \mathcal{A}$ a morphism of $\mathcal{Z}_{0}$-algebras. Suppose there exists a $\mathcal{Z}_{0}$-module $\mathcal{R}$ in $\mathcal{A}$ with support $\mu_{0} \circ \Lambda(1)$ (where $\Lambda$ is defined by (3.12)) such that

$$
\left\{\begin{array}{l}
\mathcal{R} \mathcal{R}^{*}=\mathcal{Z}_{0} \cdot \mu_{0} \circ \Lambda(1) \\
R \mu_{0}(y)=\mu_{0} \circ \Lambda(y) R \quad, \quad y \in \mathcal{Z}_{0} \otimes \mathcal{O}_{\mathbb{U}(d)}, R \in \mathcal{R}
\end{array}\right.
$$

then $\mu_{0}$ extends uniquely to a morphism $\mu: \mathcal{O}_{\mathbf{S U} \mathcal{E}} \rightarrow \mathcal{A}$, for every vector bundle $\mathcal{E} \rightarrow \widehat{Z}_{0}$ such that $(\iota, \lambda \mathcal{E}) \simeq \mathcal{R}$, by defining $\mu(\iota, \lambda \mathcal{E}):=\mathcal{R}$. 
Proof. By [27] there is a unital $\mathcal{Z}^{\rho}$-monomorphism $\mathcal{A} \hookrightarrow \mathcal{L}(\mathcal{H})$, where $\mathcal{H}$ is a Hilbert $\mathcal{Z}_{0}{ }^{\prime \prime}$-module. Thus for every vector bundle $\mathcal{E}$ with $(\iota, \lambda \mathcal{E}) \simeq \mathcal{R}$ we can regard at $\mu_{0}$ as a covariant representation of $\left(\mathcal{O}_{\mathbf{U E}}, \Lambda\right)$ with rank $\lambda \mathcal{E}$ (recall Lemma 3.8). By universality of the crossed product we can find a $\mathcal{Z}_{0}$ homomorphism $\mu: \mathcal{O}_{\text {SUE }} \rightarrow \mathcal{L}(\mathcal{H})$ having range generated by $\operatorname{Im}\left(\mu_{0}\right)$ and $\mathcal{R}$ (thus contained in $\mathcal{A}$ ).

By definition every endomorphism $\rho$ of $\mathcal{A}$ carrying weak permutation symmetry $p \mapsto \varepsilon(p)$ induces a morphism $C^{*}\left(\mathbb{P}_{\infty}\right) \rightarrow \mathcal{A}$. We now consider the finite set of characteristic functions $\left\{\chi_{i}\right\} \subset \mathcal{Z}^{\rho}$ corresponding to the decomposition of $\widehat{\mathcal{Z}}^{\rho}$ into connected components, and decompose the $C^{*}$-dynamical system $(\mathcal{A}, \rho)$ into the direct sum $\oplus_{i}\left(\mathcal{A}_{i}, \rho_{i}\right)$, where $\mathcal{A}_{i}:=\chi_{i} \cdot \mathcal{A}$ is the reduced $C^{*}$ algebra by $\chi_{i}, \rho_{i}\left(\chi_{i} a\right):=\chi_{i} \rho(a)$ for $a \in \mathcal{A}$. Each endomorphism $\rho_{i}$ carries a weak permutation symmetry $\varepsilon_{i}: p \mapsto \chi_{i} \varepsilon(p)$ if $\rho$ does. Following Doplicher and Roberts [16], we may say that a permutation symmetry of $\rho$ has dimension $d \in \mathbb{N}$ if the kernel of the morphism $C^{*}\left(\mathbb{P}_{\infty}\right) \rightarrow \mathcal{A}$ is the ideal generated by the totally antisymmetric projection in $C^{*}\left(\mathbb{P}_{d+1}\right)$. But by above considerations a more general situation may arise, namely that every permutation symmetry $\varepsilon_{i}$ has distinct dimension $d_{i}$.

Definition 6.7. Let $\rho$ be a unital endomorphism of a $C^{*}$-algebra $\mathcal{A}$ carrying weak permutation symmetry, $d: \widehat{\mathcal{Z}}^{\rho} \rightarrow \mathbb{N}$ a continuous (locally constant) map with range a finite set $\left\{d_{i}\right\}$ of positive integers indexed by the connected components of $\widehat{\mathcal{Z}}^{\rho}$. Then $\rho$ has rank $d$ if every endomorphism $\rho_{i}$ defined on the reduced $C^{*}$-algebra $\mathcal{A}_{i}$ over $i$-th connected component of $\widehat{\mathcal{Z}}^{\rho}$ has dimension $d_{i}$.

Thus the rank $d$ defines an element of the sheaf cohomology group $H^{0}\left(\widehat{\mathcal{Z}}^{\rho}, \mathbb{Z}\right)$ (i.e. a locally constant $\mathbb{Z}$-valued function on $\widehat{\mathcal{Z}}^{\rho}$ ). Canonical endomorphisms of $C^{*}$-algebras of vector bundles supply a class of examples of previously defined rank.

In order to simplify the esposition in the sequel we will restrict our attention to the case $d \in \mathbb{N}$ (with $d>1$ ). Note in fact that there is no loss of generality, thanks to above direct sum decomposition of $(\mathcal{A}, \rho)$.

Lemma 6.8. Let $\rho$ be an endomorphism of a unital $C^{*}$-algebra $\mathcal{A}$ carrying rank $d$ weak permutation symmetry $p \mapsto \varepsilon(p), p \in \mathbb{P}_{\infty}$. Then for every rank $d$ vector bundle $\mathcal{E} \rightarrow \widehat{\mathcal{Z}}^{\rho}$ there is a unique dual $\mathbf{U} \mathcal{E}$-action $\mu$ on $(\mathcal{A}, \rho)$ with $\mu(\theta)=\varepsilon$ and $\mu \circ \sigma_{G}=\rho \circ \mu$. If, furthermore, there exists in $\mathcal{A}$ a Hilbert $\mathcal{Z}^{\rho}$-bimodule $\mathcal{R} \subseteq\left[\iota, \rho^{d}\right]$ with $\mathcal{R}^{*}=\mathcal{Z}^{\rho} P_{\varepsilon, d}$, then for every rank $d$ vector bundle $\mathcal{E} \rightarrow \widehat{\mathcal{Z}}^{\rho}$ such that $(\iota, \lambda \mathcal{E}) \simeq \mathcal{R}$ the dual action $\mu$ uniquely extends to $a$ dual SUE-action by requiring $\mu(\iota, \lambda \mathcal{E})=\mathcal{R}$.

Proof. As permutation symmetry has rank $d$ there is a dual action $\mathcal{O}_{\mathbb{U}(d)} \rightarrow \mathcal{A}$ that extends in the natural way to a dual action $\mu_{0}$ of $\mathbf{U} \mathcal{E}$ on $(\mathcal{A}, \rho)$ (in fact $\mathcal{E}$ has rank $d$ ). By Lemma 6.6] we know that there is an $\mathcal{Z}^{\rho}$-morphism $\mu$ : 
$\mathcal{O}_{\text {SUE }} \rightarrow \mathcal{A}$ extending $\mu_{0}$ and such that $\mu(\iota, \lambda \mathcal{E})=\mathcal{R}$. In order to prove that $\mu$ is actually a dual action we have to verify first that

$$
\mu \circ \sigma_{\mathrm{SU} \mathcal{E}}=\rho \circ \mu \text {. }
$$

Now observe that as $\mu_{0}$ is a dual action then (6.4) is verified for elements of $\left(\sigma_{\mathbf{S U} \mathcal{E}}^{r}, \sigma_{\text {SUE }}^{r}\right)$. As $\mathcal{O}_{\mathbf{S U E}}$ is generated by $\mathcal{O}_{\mathbf{U E}}$ and $(\iota, \lambda \mathcal{E})$ it remains to verify (6.4) only for elements of $(\iota, \lambda \mathcal{E})$. By hypotesis $\mathcal{R} \subset\left[\iota, \rho^{d}\right]$ so that if $R \in \mathcal{R}$ then $\varepsilon(d, 1) R=\rho(R)$; now if $y \in(\iota, \lambda \mathcal{E})$ then $\theta(d, 1) y=\sigma_{\mathbf{S U E}}(y)$ and $\mu(\theta(d, 1) y)=\varepsilon(d, 1) \mu(y)=\mu \circ \sigma_{\mathbf{S U E}}(y)$. But $\mu(y) \in \mathcal{R} \subset\left[\iota, \rho^{d}\right]$ so that $\varepsilon(d, 1) \mu(y)=\rho \circ \mu(y)$ and $\mu \circ \sigma_{\mathbf{S U E}}(y)=\rho \circ \mu(y)$. At last we prove that $\mu\left(\sigma_{\mathbf{S U E}}^{r}, \sigma_{\mathbf{S U} \mathcal{E}}^{r+k d}\right) \subset\left(\rho^{r}, \rho^{r+k d}\right)$. If $k=0$ we know that being $\mu_{0}$ a dual action the inclusion is verified. Let $\left\{S_{L}\right\} \subset\left(\iota, \lambda \mathcal{E}^{k}\right)$ be a subset with $\sum_{L} S_{L}^{*} S_{L}=1$ (such a set is constructed by using Serre-Swan Theorem and partition functions arguments). Now if $y \in\left(\sigma_{\mathbf{S U} \mathcal{E}}^{r}, \sigma_{\mathbf{S U}}^{r+k d}\right)$ then $\mu(y)=\sum_{L} \mu_{0}\left(y \sigma_{\mathbf{S U} \mathcal{E}}^{r}\left(S_{L}^{*}\right)\right) \cdot \mu \circ$ $\sigma_{\mathbf{S U} \mathcal{E}}^{r}\left(S_{L}\right)$, with $\mu_{0}\left(y \sigma_{\mathbf{S U E}}^{r}\left(S_{L}^{*}\right)\right) \subset\left(\rho^{r+k d}, \rho^{r+k d}\right)$ and $\mu \circ \sigma_{\mathbf{S U} \mathcal{E}}^{r}\left(S_{L}\right)=\rho^{r} \circ$ $\mu\left(S_{L}\right) \subset \rho^{r}\left(\mathcal{R}^{k}\right) \subset\left(\rho^{r}, \rho^{r+k d}\right)$.

We now introduce the notion of special conjugate property in our general setting. Note that in the case $\mathcal{Z}=\mathbb{C}$ it is implemented by an isometry (see Def 1.2), that is identified as a generator of the totally antisymmetric tensor power of the Hilbert space inducing $\rho$, if we construct the crossed product in Thm. 1.3 But as we are dealing with modules of continuous sections of vector bundles the totally antisymmetric tensor power is a line bundle, that cannot in general be encoded as an isometry, unless triviality. Thus we define the special conjugate property in terms of a Hilbert bimodule in $\mathcal{A}$, corresponding to the modules of continuous sections of a line bundle.

Definition 6.9 (Special Conjugate Property). Let $\mathcal{A}$ be a unital $C^{*}$ algebra. A unital endomorphism $\rho$ having weak permutation symmetry satisfies the special conjugate property if for some $d \in \mathbb{N}, d>1$ there is a finitely generated Hilbert $\mathcal{Z}^{\rho}$-bimodule $\mathcal{R} \subset\left[\iota, \rho^{d}\right]$ (i.e. $\varepsilon(d, 1) R=\rho(R)$ for each $R \in \mathcal{R}$ ) such that

$$
\begin{gathered}
R^{*} \rho\left(R^{\prime}\right)=(-1)^{d-1} d^{-1} R^{*} R^{\prime} \quad, \quad R, R^{\prime} \in \mathcal{R} \\
\mathcal{R}^{*}=\mathcal{Z}^{\rho} P_{\varepsilon, d} .
\end{gathered}
$$

Property (6.6) forces $\mathcal{R}$ to be the module of sections of a line bundle over the spectrum $\widehat{\mathcal{Z}}^{\rho}$, and implies that the support of $\mathcal{R}$ is $P_{\varepsilon, d}$. Property (6.5) implies that for each set $\left\{R_{i}\right\}$ of normalized generators of $\mathcal{R}$ the equation

$$
\sum_{i} R_{i}^{*} \rho\left(R_{i}\right)=(-1)^{d-1} d^{-1} 1
$$


holds, in analogy with Eq. (3.10). We can more generally state our special conjugate property Def 6.9 by considering a non constant rank $d \in H^{0}\left(\widehat{\mathcal{Z}}^{\rho}, \mathbb{Z}\right)$, by performing decomposition of the system $(\mathcal{A}, \rho) \simeq \oplus_{i}\left(\mathcal{A}_{i}, \rho_{i}\right)$ according to connected components of $\widehat{\mathcal{Z}}^{\rho}$ as in Def 6.7 and by giving previous definition for each index $i$. The result is a Hilbert $\mathcal{Z}^{\rho}$-bimodule $\mathcal{R}$ in $\mathcal{A}$ with a natural decomposition $\mathcal{R} \simeq \oplus_{i} \mathcal{R}_{i} \subset \oplus_{i}\left[\iota, \rho_{i}^{d_{i}}\right]$, where each $\mathcal{R}_{i}$ satisfies 6.5] 6.6) for a fixed dimension $d_{i}$. In the sequel we will for simplicity assume $d=$ cost. We now prove unicity of the module $\mathcal{R}$ satisfying the special conjugate property.

Lemma 6.10. There is only one $\mathcal{Z}^{\rho}$-bimodule $\mathcal{R} \subset\left(\iota, \rho^{d}\right)$ satisfying (6.5[6.6).

Proof. Let $\mathcal{S} \subset\left(\iota, \rho^{d}\right)$ be another $\mathcal{Z}^{\rho}$-module satisfying Def 6.9 with a set of generators $\left\{S_{j}\right\}$. We consider a set of normalized generators $\left\{R_{i}\right\}$ for $\mathcal{R}$ and define $z_{i j}:=R_{i}^{*} S_{j} \in \mathcal{Z}$ for each $i, j$. In order to prove the lemma it will suffice to show that $z_{i j} \in \mathcal{Z}^{\rho}$. At this purpose observe that for each $R \in \mathcal{R}$ the identity $R_{i}^{*} \rho(R)=(-1)^{d-1} d^{-1} R_{i}^{*} R$ holds; so that, multiplying on the left by $R_{i}$ and summing over $i$ we get

$$
P_{\varepsilon, d} \rho(R)=(-1)^{d-1} d^{-1} R .
$$

In the same way, for each $S \in \mathcal{S}$, we obtain $P_{\varepsilon, d} \rho(S)=(-1)^{d-1} d^{-1} S$. Now observe that $R_{k} R_{i}^{*}=f_{i k} P_{\varepsilon, d}$, where $f_{i k} \in \mathcal{Z}^{\rho}$, so that $R_{k} z_{i j}=z_{i j} R_{k}=$ $R_{i}^{*} S_{j} R_{k}=f_{i k} P_{\varepsilon, d} S_{j}=f_{i k} S_{j}$. Thus $\rho\left(R_{k}\right) \rho\left(z_{i j}\right)=f_{i k} \rho\left(S_{j}\right)$; multiplying this last identity on the left by $P_{\varepsilon, d}$ and applying (6.8) (and analogue) for $R_{k}, S_{j}$ we obtain

$$
(-1)^{d-1} d^{-1} R_{k} \rho\left(z_{i j}\right)=(-1)^{d-1} d^{-1} f_{i k} S_{j}=(-1)^{d-1} d^{-1} R_{k} z_{i j}
$$

and $\rho\left(z_{i j}\right)^{*} R_{k}^{*}=z_{i j}^{*} R_{k}^{*}$. Multiplying on the right by $R_{k}$ and summing over $k$ we get $\rho\left(z_{i j}\right)^{*}=z_{i j}^{*}$.

The first Chern class in $H^{2}\left(\widehat{\mathcal{Z}}^{\rho}, \mathbb{Z}\right)$ (sheaf cohomology) of the line bundle having $\mathcal{R}$ as module of continuous sections is a complete invariant of $\mathcal{R}$ as a Hilbert $\mathcal{Z}^{\rho}$-module; unicity established in Lemma 6.10 implies that it is actually an invariant of $\rho$. We denote it by $c_{1}(\rho)$.

We now give an application of [16 Thm. 4.3 to compute the rank of weak permutation symmetry $p \mapsto \varepsilon(p)$ for an endomorphism $\rho$ satisfying the special conjugate property Def6.9. This result states that if $\phi: \mathcal{A} \rightarrow \mathcal{A}$ is a left inverse for $\rho$ with $\phi(\varepsilon)=\lambda 1, \lambda \in \mathbb{C}$ then $d$ is recovered by the formula $\lambda= \pm d^{-1} 1$, and $\lambda \in\{0\} \cup\left\{ \pm k^{-1}, k \in \mathbb{Z}-\{0\}\right\}$.

Lemma 6.11. Let $\rho$ be an endomorphism of a unital $C^{*}$-algebra $\mathcal{A}$ satisfying the special conjugate property Def [6.9. Then $\rho$ has weak permutation symmetry of rank $d$. 
Proof. We consider a normalized (finite) set $\left\{R_{i}\right\}$ of generators of $\mathcal{R}$, and define the left inverse

$$
\phi(a):=\sum_{i} R_{i}^{*} \rho^{d-1}(a) R_{i}, \quad a \in \mathcal{A} .
$$

In order to apply [16] Thm. 4.3 we have to compute explicity $\phi(\varepsilon)$. At this purpose note that $\mathbb{S}^{d-1}(1,1)=(d-1,1)(d-1)$, so that $\rho^{d-1}(\varepsilon)=\varepsilon(d-$ $1,1) \varepsilon(d, 1)$. Thus $\phi(\varepsilon)=\sum_{i} R_{i}^{*} \varepsilon(d-1,1) \varepsilon(d, 1) R_{i}$. Now, $\varepsilon(d, 1) R_{i}=\rho\left(R_{i}\right)$ (as $\left.\mathcal{R} \subset\left[\iota, \rho^{d}\right]\right)$ and $\varepsilon(1, d-1) P_{\varepsilon, d}=\operatorname{sign}(1, d-1) P_{\varepsilon, d}=(-1)^{d-1} P_{\varepsilon, d}$, so that $\varepsilon(1, d-1) R_{i}=(-1)^{d-1} R_{i}$. Thus $\phi(\varepsilon)=(-1)^{d-1} \sum_{i} R_{i}^{*} \rho\left(R_{i}\right)$. By applying (6.7) we find $\phi(\varepsilon)=d^{-1}$.

Definition 6.12. A unital endomorphism $\rho$ of $\mathcal{A}$ is weak special if satisfies the special conjugate property Def 6.9 The class of $\rho$ is

$$
c(\rho):=c_{0}(\rho) \oplus c_{1}(\rho) \in H^{0}\left(\widehat{\mathcal{Z}}^{\rho}, \mathbb{Z}\right) \oplus H^{2}\left(\widehat{\mathcal{Z}}^{\rho}, \mathbb{Z}\right),
$$

where $c_{0}(\rho)$ is the rank of weak permutation symmetry, and $c_{1}(\rho)$ is the Chern class of the Hilbert $\mathcal{Z}^{\rho}$-bimodule $\mathcal{R}$ introduced in Def $6.9, \rho$ is said special when furthermore permutation symmetry Def6.3 holds.

Note that classical special conjugate property Def 1.2 is satisfied if and only if $c_{1}(\rho)$ vanishes and $c_{0}(\rho)$ is a constant map. In this case $\mathcal{R}$ is generated by a partial isometry with support $P_{\varepsilon, d}$ as a Hilbert $\mathcal{Z}^{\rho}$-module in $\mathcal{A}$.

Example 6.3. Let $\mathcal{E} \rightarrow X$ be a rank $d$ vector bundle, $G \subseteq \mathbf{S U E}$. Then $\sigma_{G}$ is special in the sense of Def. 6.12 In fact $\sigma_{G}$ carries permutation symmetry and satisfies special conjugate property Def. [6.9] implemented by the module of continuous sections of $d$-fold exterior power $\lambda \mathcal{E}$ (see Sec [3). Thus $c\left(\sigma_{G}\right)=$ $d \oplus c_{1}(\mathcal{E})$, where $c_{1}(\mathcal{E})$ is the first Chern class of $\mathcal{E}$.

Example 6.4. More generally, let $\mathcal{M} \simeq \widehat{\mathcal{E}} \otimes_{C(X)} C\left(X^{\prime}\right)$ be a noncommutative pullback, $G \subseteq \mathbf{S U E}$ a closed group acting on $\mathcal{M}$ by $C\left(X^{\prime}\right)$-bimodule maps with $\mathcal{O}_{\mathcal{M}}^{G} \subseteq \mathcal{B}_{\mathcal{M}}$. By Prop 6.4 follows that the shift $\tau_{G}(t):=1 \otimes t, t \in\left(\mathcal{M}^{r}, \mathcal{M}^{s}\right)_{G}$ defined on $\mathcal{O}_{\mathcal{M}}^{G}$ carries permutation symmetry; furthermore being $G \subseteq \mathbf{S U E}$ we find that $\tau_{G}$ satisfies the special conjugate property implemented by $(\iota, \lambda \mathcal{E}) \subseteq$ $\left(\iota, \tau_{G}^{d}\right)$, so that it is a special endomorphism in the sense of Def 6.12

\section{Crossed Products by Special Endomorphisms.}

We proceed in this section to the construction of crossed products by special endomorphisms. The first step is the following basic result, that is consequence of Lemma 6.8 Lemma 6.11.

Theorem 7.1. Let $\rho$ be a weak special endomorphism of a unital $C^{*}$-algebra $\mathcal{A}$ with class $c(\rho)=c_{0}(\rho) \oplus c_{1}(\rho)$. Then every vector bundle $\mathcal{E} \rightarrow \widehat{\mathcal{Z}}^{\rho}$ with rank $c_{0}(\rho)$ and with first Chern class $c_{1}(\rho)$ induces a dual action $\widehat{\mathbf{S U E}} \rightarrow \widehat{\rho} \subset$ end $\mathcal{A}$. 
Previous theorem allows to construct the crossed product by the dual SUE action, that we denote by $\mathcal{A} \rtimes_{\rho} \widehat{\mathrm{SUE}}$, satisfying the following properties:

- $\widehat{\mathcal{E}}$ is contained in $\mathcal{A} \rtimes_{\rho} \widehat{\mathbf{S U E}}$ as a $\mathcal{Z}^{\rho}$-Hilbert bimodule with support 1 and induces an endomorphism $\sigma_{\mathcal{E}}$ on $\mathcal{A} \rtimes_{\rho} \widehat{\mathrm{SUE}}$;

- there is an action $\alpha$ of SUE on $\mathcal{A} \rtimes_{\rho} \widehat{\mathbf{S U E}}$, coinciding with the natural action of SUE on $\widehat{\mathcal{E}}$ (so that $\widehat{\mathcal{E}}$ is $\alpha$-stable);

- there is a unital inclusion $\mathcal{A} \hookrightarrow \mathcal{A} \rtimes_{\rho} \widehat{\mathrm{SUE}}$ such that $\mathcal{A}=\left(\mathcal{A} \rtimes_{\rho} \widehat{\mathrm{SUE}}\right)^{\alpha}$, and $\sigma_{\mathcal{E}}$ restricts to $\rho$ on $\mathcal{A}$.

- $\mathcal{A}$ and $\widehat{\mathcal{E}}$ generate $\mathcal{A} \rtimes_{\rho} \widehat{\mathrm{SUE}}$ as a $C^{*}$-algebra.

Let now $\mathcal{J}$ be a closed ideal of $\mathcal{A} \rtimes_{\rho} \widehat{\mathrm{SUE}}$. We denote by $\mathcal{F}_{\mathcal{J}}$ the quotient of $\mathcal{A} \rtimes_{\rho} \widehat{\mathbf{S U E}}$ by $\mathcal{J}$ and by $\eta_{\mathcal{J}}: \mathcal{A} \rtimes_{\rho} \widehat{\mathrm{SUE}} \rightarrow \mathcal{F}_{\mathcal{J}}$ the quotient morphism. It is clear that the closed group

$$
G_{\mathcal{J}}:=\left\{g \in \mathbf{S U E}: \alpha_{g}(\mathcal{J})=\mathcal{J}\right\}
$$

acts by automorphisms on $\mathcal{F}_{\mathcal{J}}$. With an abuse of notation, we denote by $\alpha: G_{\mathcal{J}} \rightarrow$ aut $\mathcal{F}_{\mathcal{J}}$ the corresponding action, so that $\eta_{\mathcal{J}} \circ \alpha_{g}=\alpha_{g} \circ \eta_{\mathcal{J}}$ for every $g \in G_{\mathcal{J}}$. Suppose now $\mathcal{A} \cap \mathcal{J}=\{0\}$. Then the following elementary properties hold:

- $\eta_{\mathcal{J}}$ restricts to a unital monomorphism from $\mathcal{A}$ into $\mathcal{F}_{\mathcal{J}}$.

- $\mathcal{J}$ has trivial intersection with $\widehat{\mathcal{E}}$ (in fact if $\psi \in \widehat{\mathcal{E}} \cap \mathcal{J}$ then $\psi^{*} \psi \in \mathcal{A} \cap \mathcal{J}=$ $\{0\})$. So that $\eta_{\mathcal{J}}$ restricts to a unital monomorphism $\mathcal{O}_{\mathcal{E}} \hookrightarrow \mathcal{F}_{\mathcal{J}}$.

- $\widehat{\mathcal{E}} \simeq \eta_{\mathcal{J}}(\widehat{\mathcal{E}})$ is contained in $\mathcal{F}_{\mathcal{J}}$ as a $\mathcal{Z}^{\rho}$-Hilbert bimodule with support 1 , and induces an inner endomorphism $\sigma_{\mathcal{J}}$ on $\mathcal{F}_{\mathcal{J}}$. It is clear that $\eta_{\mathcal{J}} \circ \sigma_{\mathcal{E}}=$ $\sigma_{\mathcal{J}} \circ \eta_{\mathcal{J}}$

The following class of ideals of $\mathcal{A} \rtimes_{\rho} \widehat{\mathrm{SUE}}$ plays a basic role in our construction.

Definition 7.2. With above introduced notations, a maximal $\mathcal{A}$-module ideal is a closed ideal $\mathcal{J}$ of $\mathcal{A} \rtimes_{\rho} \widehat{\mathrm{SUE}}$ such that $\mathcal{A} \cap \mathcal{J}=\{0\},\left(\mathcal{F}_{\mathcal{J}}\right)^{G_{\mathcal{J}}}=\eta_{\mathcal{J}}(\mathcal{A})$ and $\eta_{\mathcal{J}}(\mathcal{A})^{\prime} \cap \mathcal{F}_{\mathcal{J}}=\eta_{\mathcal{J}}(\mathcal{Z})$.

Remark 7.1. If $\mathcal{J}$ is a maximal $\mathcal{A}$-module ideal then the centre $\mathcal{C}_{\mathcal{J}}$ of $\mathcal{F}_{\mathcal{J}}$ is isomorphic to $\mathcal{Z}^{\rho}$. In fact if $c \in \mathcal{C}_{\mathcal{J}}$ then $\sigma_{\mathcal{J}}(c)=c$ and $c \in \eta_{\mathcal{J}}(\mathcal{A})^{\prime} \cap \mathcal{F}_{\mathcal{J}}=$ $\eta_{\mathcal{J}}(\mathcal{Z})$. Let us now denote by $\mathcal{C}$ the centre of $\mathcal{A} \rtimes_{\rho} \widehat{\mathbf{S U E}}$; then $\eta_{\mathcal{J}}$ restricts to a $\mathcal{Z}^{\rho}$-morphism $\phi_{\mathcal{J}}: \mathcal{C} \rightarrow \mathcal{Z}^{\rho}$, i.e. (by Gel'fand) a continuous section 
$s_{\mathcal{J}}: \widehat{\mathcal{Z}}^{\rho} \rightarrow \widehat{\mathcal{C}}$ is defined, where $\widehat{\mathcal{C}}$ is the spectrum of $\mathcal{C}$. The maximal $\mathcal{A}$ module ideals of $\mathcal{A} \rtimes_{\rho} \widehat{\mathbf{S U E}}$ correspond bijectively to such continuous sections by assigning $\mathcal{J}:=\operatorname{ker} \phi_{\mathcal{J}} \cdot\left(\mathcal{A} \rtimes_{\rho} \widehat{\mathrm{SUE}}\right)$, as can be deduced by the construction in [42].

Lemma 7.3. Every automorphism of $\mathcal{A} \rtimes_{\rho} \widehat{\mathrm{SUE}}$ leaving $\mathcal{A}$ pointwise invariant (in particular automorphisms coming from $\mathbf{S U E}$-action) carries maximal $\mathcal{A}$ module ideals into maximal $\mathcal{A}$-module ideals.

Proof. Let $\alpha$ be an automorphism of $\mathcal{A} \rtimes_{\rho} \widehat{\mathrm{SUE}}$ leaving $\mathcal{A}$ pointwise invariant. Then $\alpha(\mathcal{J})$ is an ideal and for $b \in \alpha(\mathcal{J}) \cap \mathcal{A}$ we find $b=\alpha(b)=\alpha^{-1}(b) \in$ $\mathcal{J} \cap \mathcal{A}=\{0\}$. Furthermore if $b^{\prime} \in \mathcal{A} \rtimes_{\rho} \widehat{\mathbf{S U E}}$ and $\left[b^{\prime}, a\right] \in \alpha(\mathcal{J})$ for each $a \in \mathcal{A}$ then $\alpha^{-1}\left(b^{\prime}\right) \in \mathcal{Z}+\mathcal{J}$, i.e. $b^{\prime} \in \mathcal{Z}+\alpha(\mathcal{J})$. Finally, by the identity $G_{\alpha(\mathcal{J})}=\alpha G_{\mathcal{J}} \alpha^{-1}$ follows that $\left(\mathcal{F}_{\alpha(\mathcal{J})}\right)^{G_{\alpha(\mathcal{J})}} \simeq\left(\mathcal{F}_{\mathcal{J}}\right)^{G_{\mathcal{J}}}=\eta_{\mathcal{J}}(\mathcal{A})$.

We now give the following result in order to prove existence of maximal $\mathcal{A}$-module ideals.

Theorem 7.4. Let $\mathcal{A} \rtimes_{\mu} \widehat{G}$ be a crossed product by a dual action, with $G \subseteq \mathbf{S U E}$ locally trivial. If $\rho$ satisfies permutation symmetry Def 6.3 then the following properties hold:

- $\mathcal{A}^{\prime} \cap\left(\mathcal{A} \rtimes_{\mu} \widehat{G}\right)$ is generated as a $C^{*}$-algebra by $\mathcal{Z}$ and the centre $\mathcal{C}$ of $\mathcal{A} \rtimes_{\mu} \widehat{G}$

- if $\mu(\widehat{G})=[\rho]$ then $\mathcal{A}^{\prime} \cap\left(\mathcal{A} \rtimes_{\mu} \widehat{G}\right)=\mathcal{Z}$;

- there is an inclusion of $C(X)$-algebras $\mathcal{C} \hookrightarrow C(\mathcal{G})$, where $\mathcal{G}$ is the spectral bundle of $G$.

Proof.

- In order to economize in notations we put $\mathcal{B}:=\mathcal{A} \rtimes_{\mu} \widehat{G}$. As a first step we assume that $\mathcal{E}$ is trivial (thus $\widehat{\mathcal{E}}=H \cdot \mathcal{Z}^{\rho}$, where $H \simeq \mathbb{C}^{d}$ ), and $G$ is a closed subgroup of $\mathbb{S U}(d)$, so that we can proceede in a order of ideas similar to [17], Lemma 5.3. Now $\mathcal{A}^{\prime} \cap \mathcal{B}$ is $G$-stable and is generated as a $C^{*}$-algebra by the set of irreducible tensors of the type $\left\{T_{1}, \ldots T_{n}\right\}$ satisfying the relations

$$
\alpha_{g}\left(T_{j}\right)=\sum_{j}^{N} T_{j} u_{i j}(g),
$$

where $g \in G$ and $u_{i j} \in \mathbb{C}$ are matrix elements in some irreducible representation of $G$. Let now $R \in H^{d}$ be a generator of the totally antisymmetric tensor power of $H$. By hypothesis $R$ is $G$-invariant and belongs to 
$\left[\iota, \rho^{d}\right] \subset \mathcal{A}$. Now we can find $p, n \in \mathbb{N}$ such that for $\left\{\psi_{i}\right\}$ orthonormal basis of $H^{n}$, the tensor $\left\{R^{* p} \psi_{i}\right\}$ trasform like $\left\{T_{i}\right\}$. Thus $W:=\sum_{i} R^{p} T_{i} \psi_{i}^{*}$ is $G$-invariant and belongs to $\mathcal{A}$. Furthermore $T_{i}=R^{* p} W \psi_{i}$, where $W \in\left(\rho^{n}, \rho^{p d}\right), \psi \in H^{n} \subset\left(\iota, \sigma_{\mathcal{E}}^{n}\right), R^{* p} \in\left(\sigma_{\mathcal{E}}^{p d}, \iota\right)$. Thus $W$ is a finite sum of terms of the type $\rho^{p d}(z) \bar{W}$, where $z \in \mathcal{Z}$ and $\bar{W} \in\left[\rho^{n}, \rho^{p d}\right]$. In order to simplify our notations we assume that $W=\rho^{p d}(z) \bar{W}$. So that we obtain $T_{i}=z R^{* p} \bar{W} \psi_{i}$ and define $\bar{T}_{i}:=R^{* p} \bar{W} \psi_{i}$. We now prove that $\sigma_{\mathcal{E}}\left(\bar{T}_{i}\right)=\bar{T}_{i}$, implying $\bar{T}_{i} \in \mathcal{B} \cap \mathcal{B}^{\prime}$. In fact by permutation symmetry we obtain $\sigma_{\mathcal{E}}\left(\bar{T}_{i}\right)=\rho\left(R^{*}\right)^{p} \rho(\bar{W}) \sigma_{\mathcal{E}}\left(\psi_{i}\right)=R^{* p} \varepsilon(1, p d) \cdot \varepsilon(p d, 1) \bar{W} \varepsilon(1, n)$. $\varepsilon(n, 1) \psi_{i}=\bar{T}_{i}$. Thus our first step is proved.

Let us now consider the general case. We regard at $\mathcal{B}$ as the $C^{*}$-algebra of sections of an upper semicontinuous bundle over $\widehat{\mathcal{Z}}^{\rho}$. Let $x \in \widehat{\mathcal{Z}}^{\rho}, U$ an open neighborhood of $x$ trivializing $\mathcal{E}, G$. Then $\left.G\right|_{U} \simeq U \times G_{0}$, with $G_{0} \subseteq \mathbb{S U}(d)$, and $G_{0}$ acts on $\mathcal{B}_{U}:=\left.\mathcal{B}\right|_{U}$ in such a way that $\mathcal{B}_{U}^{G_{0}}=\mathcal{A}_{U}$. If $\left\{T_{i}\right\}$ is an irreducible tensor in $\mathcal{A}_{U}$ as above there is a closed $V \subset U$ and $\lambda \in \mathcal{Z}^{\rho}$ with $\left.\lambda\right|_{V}=1,\left.\lambda\right|_{\hat{\mathcal{Z}}^{\rho}-U}=0$. With above notations, we observe that $\lambda W \in\left(\rho^{n}, \rho^{p d}\right)=\rho^{n d}(\mathcal{Z})\left[\rho^{n}, \rho^{p d}\right]$, and conclude with the argument of previous step that $\sigma_{\mathcal{E}}\left(\lambda \bar{T}_{i}\right)=\lambda \bar{T}_{i}$. Thus $\lambda T_{i} \in\left(\mathcal{B} \cap \mathcal{B}^{\prime}\right) \vee \mathcal{Z}$ for every $\lambda$ as above.

We now pick a finite open cover $\left\{U_{k}\right\}$ trivializing $\mathcal{E}, G$ with a subordinate partition of unity $\left\{\lambda_{k}\right\}$. If $b \in \mathcal{A}^{\prime} \cap \mathcal{B}$ there is $\epsilon>0$ and a linear combination $T_{k} \in \mathcal{B}_{U_{k}}$ of irreducible tensors, with $\left\|\lambda_{k} b-\lambda_{k} T_{k}\right\|<\epsilon$. Note that by above argument $\lambda_{k} T_{k} \in\left(\mathcal{B} \cap \mathcal{B}^{\prime}\right) \vee \mathcal{Z}$. Thus $\left\|b-\sum_{k} \lambda_{k} T_{k}\right\|<$ $\epsilon$ and $b$ is aproximated by elements of $\left(\mathcal{B} \cap \mathcal{B}^{\prime}\right) \vee \mathcal{Z}$.

- By the previous point follows that $\mathcal{C}$ is generated by elements of the type $\sum_{k} f_{k} \bar{T}_{k}$, where $f_{k} \in C(X)$ and $\bar{T}_{k}:=\lambda_{k} R^{* p} \bar{W} \psi_{k}$ are the central irreducible tensors transforming according (7.2), and smoothed by the cutoff functions $\lambda_{k} \in C(X)$. If $\mu(\widehat{G})=[\rho]$ then $\bar{W} \in\left(\mathcal{E}^{n}, \mathcal{E}^{p d}\right)_{G}$, and $\bar{T}_{k} \in\left(\mathcal{E}^{p d}, \iota\right) \cdot\left(\mathcal{E}^{n}, \mathcal{E}^{p d}\right)_{G} \cdot\left(\iota, \mathcal{E}^{n}\right)=C(X)$.

- By general facts (Lemma 4.1 in 42 ), it can be deduced that $\mathcal{C}$ corresponds to a continuous field over $X$ with fibre the $C^{*}$-algebra of continuous maps over the homogeneous space $H_{x} / G_{0}$, where $G_{0}$ is the fibre of $G$ and $H_{x}$ is the stabilizer of some element $\omega_{x}$ of the spectrum $\widehat{\mathcal{C}}$ of $\mathcal{C}$ (so that there is a continuous surjective map $p: \widehat{\mathcal{C}} \rightarrow X$ with $\left.p^{-1}(x) \simeq H_{x} / G_{0}\right)$. The $G$-action on $\mathcal{C}$ restricts to an ergodic $G_{0}$-action by right traslation $\omega, g \mapsto \omega \cdot g$ on every $H_{x} / G_{0}$. It is clear that the characterization of $\mathcal{C}$ as a continuous field is not canonical, depending on the choice of a family $\left\{\omega_{x}\right\}_{x \in X} \subseteq \widehat{\mathcal{C}}$. The vector field associated to $\bar{T}_{i}$ can be explicitely written, as by (7.2), as

$$
\widehat{T}_{i}\left(\omega_{x_{0}} \cdot g\right)=\sum_{k}^{N} \omega_{x_{0}}\left(\bar{T}_{k}\right) \widehat{u}_{i k}\left(x_{0}, g\right)
$$


where $\widehat{u}_{i k} \in C(\mathcal{G})$ are the Gel'fand transforms of $\lambda_{k} u_{i k}$ defined as in previous points, so that $g \mapsto \widehat{T}_{i}\left(\omega_{x_{0}} \cdot g\right)$ is a continuous map (say $T_{i, x_{0}}$ ) over $G_{0}$. The form of (7.3) depends on the choice of the family $\left\{\omega_{x}\right\}$; we will make an appropriate choice in the sequel. Now, $\widehat{T}_{i}\left(\omega_{0} \cdot g\right)=F_{i}\left(x_{0}, g\right)$, where $F_{i}:=\sum_{k} \omega_{0}\left(T_{k}\right) u_{i k} \in C(\mathcal{G})$. In order to prove that $\mathcal{C}$ is a subfield of $C(\mathcal{G})$ it suffice to verify (according to [12]) that for each $\varepsilon>0$ there is a neighborhood $U$ of $x_{0}$ such that $\sup _{U}\left\|T_{i, x}-F_{i}(x, \cdot)\right\|<\varepsilon$. We now pick a neighborhood $U^{\prime} \subseteq \widehat{\mathcal{C}}$ of $\omega_{0}$ such that $\sup _{\omega \in U^{\prime}}\left|\omega\left(\bar{T}_{k}\right)-\omega_{x_{0}}\left(\bar{T}_{k}\right)\right|<\varepsilon$, and estimate

$$
\begin{aligned}
\left\|T_{i, x}-F_{i}(x, \cdot)\right\| & =\sup _{g}\left|\widehat{T}_{i}\left(\omega_{x} \cdot g\right)-\sum_{k}^{N} \omega_{x_{0}}\left(\bar{T}_{k}\right) \widehat{u}_{i k}(x, g)\right| \\
& \leq \sum_{k}^{N}\left|\omega_{x}\left(\bar{T}_{k}\right)-\omega_{x_{0}}\left(\bar{T}_{k}\right)\right|\left\|\widehat{u}_{i k}\right\| \\
& <C \varepsilon
\end{aligned}
$$

for every $x \in p\left(U^{\prime}\right)$, where $C \in \mathbb{R}^{+}$, having chosen $\omega_{x} \in U^{\prime}$. Thus $T_{i} \in C(\mathcal{G})$.

Corollary 7.5. A maximal $\mathcal{A}$-module ideal always exists in $\mathcal{A} \rtimes_{\rho} \widehat{\mathbf{S U E}}$.

Proof. By previous theorem and Lemma 4.2 of [42] the center of $\mathcal{A} \rtimes_{\rho} \widehat{\mathbf{S U E}}$ is an homogeneous $\mathbf{S U E} \mathcal{E}$-bundle, i.e. admits a continuous section with the corresponding $\mathcal{Z}^{\rho}$-morphism $\phi: \mathcal{C} \rightarrow \mathcal{Z}^{\rho}$. Thus we can apply Theorem 4.6 of [42, establishing the existence of the maximal $\mathcal{A}$-module ideal $\mathcal{J}:=\operatorname{ker} \phi$. $\left(\mathcal{A} \rtimes_{\rho} \widehat{\mathbf{S U E}}\right)$.

We now investigate dependence of the system $\left(\mathcal{F}_{\mathcal{J}}, G_{\mathcal{J}}, \mathcal{E}\right)$ on the maximal $\mathcal{A}$-module ideal $\mathcal{J}$, establishing a unicity result.

Lemma 7.6. Let $\mathcal{I}, \mathcal{J}$ be maximal $\mathcal{A}$-module ideals with corresponding quotients $\mathcal{F}_{\mathcal{I}}, \mathcal{F}_{\mathcal{J}}$. There is $u \in \mathbf{S U E}$ and an isomorphism $\alpha_{\mathcal{I} \mathcal{J}}: \mathcal{F}_{\mathcal{I}} \rightarrow \mathcal{F}_{\mathcal{J}}$ such that the following diagram is commutative

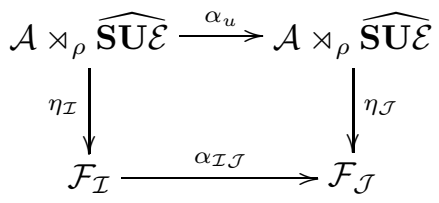

with $\alpha_{u}(\mathcal{I})=\mathcal{J}$. So that $\mathbf{S U E}$-action on the set of maximal $\mathcal{A}$-module ideals is transitive. 
Proof. By Lemma 6.5 we obtain isomorphisms of strict tensor $C^{*}$-categories $[\rho] \simeq \widehat{G}_{\mathcal{I}} \simeq \widehat{G}_{\mathcal{J}}$. Thus by Lemma 5.12 we deduce that $\mathcal{F}_{\mathcal{I}}\left(\mathcal{F}_{\mathcal{J}}\right)$ is the universal crossed product of $\mathcal{A}$ by the dual $G_{\mathcal{I}}$-action $\left(G_{\mathcal{J}}\right.$-action). By universality of the crossed product by a dual action, Lemma $\left[7.3\right.$ and the isomorphism $\widehat{G}_{\mathcal{I}} \simeq \widehat{G}_{\mathcal{J}}$, we obtain that there is an isomorphism $\alpha_{\mathcal{I} \mathcal{J}}: \mathcal{F}_{\mathcal{I}} \rightarrow \mathcal{F}_{\mathcal{J}}$, intertwining actions by $G_{\mathcal{I}}, G_{\mathcal{J}}$ and inner endomorphisms $\sigma_{\mathcal{I}}, \sigma_{\mathcal{J}}$ induced by the Hilbert $\mathcal{Z}^{\rho_{-}}$ bimodules $\eta_{\mathcal{I}}(\widehat{\mathcal{E}}), \eta_{\mathcal{J}}(\widehat{\mathcal{E}})$. In particular $\alpha_{\mathcal{I} \mathcal{J}} \circ \eta_{\mathcal{I}}(a)=\eta_{\mathcal{J}} \circ \alpha_{\mathcal{I} \mathcal{J}}(a)$ for $a \in \mathcal{A}$, and $\alpha_{\mathcal{I} \mathcal{J}}\left(\sigma_{\mathcal{I}}^{r}, \sigma_{\mathcal{I}}^{s}\right)=\left(\sigma_{\mathcal{J}}^{r}, \sigma_{\mathcal{J}}^{s}\right)$. Thus the equality

$$
\alpha_{\mathcal{I} \mathcal{J}}\left(\sigma_{G_{\mathcal{I}}}^{r}, \sigma_{G_{\mathcal{I}}}^{s}\right)=\left(\sigma_{G_{\mathcal{J}}}^{r}, \sigma_{G_{\mathcal{J}}}^{s}\right)
$$

holds (as $\left.\left(\sigma_{G_{\mathcal{I}}}^{r}, \sigma_{G_{\mathcal{I}}}^{s}\right)=\left(\sigma_{\mathcal{I}}^{r}, \sigma_{\mathcal{I}}^{s}\right) \cap \eta_{\mathcal{I}}(\mathcal{A})\right)$. We now consider the unitary $u \in \mathbf{U} \mathcal{E}$ obtained by the map $\eta_{\mathcal{J}}^{-1} \circ \alpha_{\mathcal{I} \mathcal{J}} \circ \eta_{\mathcal{I}}$ applied to elements of $\widehat{\mathcal{E}} \subset \mathcal{A} \rtimes_{\rho} \widehat{\mathrm{SUE}}$. Now, $\widehat{u}$ is the identity on $(\iota, \lambda \mathcal{E}) \subseteq\left[\iota, \rho^{d}\right] \subseteq \mathcal{A}$, so that $u \in$ SUE . Thus $\widehat{u}$ extends to an automorphism $\alpha_{u}$ of $\mathcal{A} \rtimes_{\rho} \widehat{\mathbf{S U E}}$. In order to prove the lemma it now suffice to prove that $\alpha_{u}(\mathcal{I})=\mathcal{J}$. Now, we find that $\eta_{\mathcal{J}} \circ \alpha_{u}=\alpha_{\mathcal{I} \mathcal{J}} \circ \eta_{\mathcal{I}}$. Thus in particular $\eta_{\mathcal{J}} \circ \alpha_{u}(\mathcal{I})=\alpha_{\mathcal{I} \mathcal{J}} \circ \eta_{\mathcal{I}}(\mathcal{I})=\{0\}$, and by using the same argument for $\alpha_{\mathcal{J} \mathcal{I}}$ we obtain $\alpha_{u}(\mathcal{I})=\mathcal{J}$.

Lemma 7.7. For every maximal $\mathcal{A}$-module ideal $\mathcal{J}$ the equality $G_{\mathcal{J}}=S\left(\widehat{\mathcal{Z}}^{\rho}, \mathcal{G}_{\mathcal{J}}\right)$ holds, where $\mathcal{G}_{\mathcal{J}} \rightarrow \widehat{\mathcal{Z}}^{\rho}$ is the spectral bundle spanned by $G_{\mathcal{J}}$. Furthermore groups $G_{\mathcal{I}}, G_{\mathcal{J}}$ corresponding to maximal $\mathcal{A}$-module ideals $\mathcal{I}, \mathcal{J}$ are conjugate, i.e. there is $u \in \mathbf{S U E}$ such that $u G_{\mathcal{I}} u^{*}=G_{\mathcal{J}}$.

Proof. We proved that $\mathcal{F}_{\mathcal{J}} \simeq \mathcal{A} \rtimes_{\mu} \widehat{G}_{\mathcal{J}}$, thus by Prop. 5.11 we obtain $S\left(\widehat{\mathcal{Z}}^{\rho}, \mathcal{G}_{\mathcal{J}}\right)=$ $\operatorname{aut}_{\mathcal{A}}\left(\mathcal{F}_{\mathcal{J}}, \sigma_{\mathcal{J}}\right)$. Thus if $u \in S\left(\widehat{\mathcal{Z}}^{\rho}, \mathcal{G}_{\mathcal{J}}\right)$ then diagram (7.4) commutes with $\mathcal{I}=\mathcal{J}$, so that $u \in G_{\mathcal{J}}$ and $G_{\mathcal{J}}=S\left(\widehat{\mathcal{Z}}^{\rho}, \mathcal{G}_{\mathcal{J}}\right)$. Finally, if $\alpha_{u}(\mathcal{I})=\mathcal{J}$, $u \in \mathbf{S U E}$, the automorphism $\widehat{u}$ of $\mathcal{O}_{\mathcal{E}}$ restricts by (7.5) to an isomorphism $\mathcal{O}_{G_{\mathcal{I}}} \simeq \mathcal{O}_{G_{\mathcal{J}}} ;$ thus by applying Cor 3.7 we deduce that $u G_{\mathcal{I}} u^{*}=G_{\mathcal{J}}$.

Previously established unicity allows to introduce the notations

$$
\begin{gathered}
\mathcal{A} \rtimes^{\mathcal{E}} \widehat{\rho}:=\mathcal{F}_{\mathcal{J}} \\
G:=G_{\mathcal{J}}
\end{gathered}
$$

emphasizing independence (up to isomorphism) by the choice of a maximal $\mathcal{A}$ module ideal $\mathcal{J}$. By previous results we deduce the following

Theorem 7.8. Let $\mathcal{A}$ be a unital $C^{*}$-algebra with centre $\mathcal{Z}, \rho$ a special endomorphism of $\mathcal{A}$ with class $c(\rho)=c_{0}(\rho) \oplus c_{1}(\rho), \mathcal{E} \rightarrow \widehat{\mathcal{Z}}^{\rho}$ a vector bundle with rank $c_{0}(\rho)$ and first Chern class $c_{1}(\rho)$. Then there is a unique $C^{*}$-algebra $\mathcal{A} \rtimes^{\mathcal{E}} \widehat{\rho}$ containing the Hilbert $\mathcal{Z}^{\rho}$-bimodule $\widehat{\mathcal{E}}$ of continuous sections of $\mathcal{E}$, and a strongly continuous action $\alpha$ of a closed group $G \subseteq \mathbf{S U E}$ on $\mathcal{A} \rtimes^{\mathcal{E}} \hat{\rho}$, satisfying the following properties: 
- $\alpha$ coincides with the natural $G$-action on $\widehat{\mathcal{E}}$;

- there is a unital inclusion $\mathcal{A} \hookrightarrow \mathcal{A} \rtimes^{\mathcal{E}} \widehat{\rho}$, with $\mathcal{A}=\left(\mathcal{A} \rtimes^{\mathcal{E}} \widehat{\rho}\right)^{\alpha}$;

- $\left.\sigma_{\mathcal{E}}\right|_{\mathcal{A}}=\rho$, where $\sigma_{\mathcal{E}}$ is the inner endomorphism induced by $\widehat{\mathcal{E}} \subset \mathcal{A} \rtimes^{\mathcal{E}} \widehat{\rho}$;

- $\mathcal{A}$ and $\widehat{\mathcal{E}}$ generate $\mathcal{A} \rtimes^{\mathcal{E}} \widehat{\rho}$ as a $C^{*}$-algebra, $\mathcal{A}^{\prime} \cap\left(\mathcal{A} \rtimes^{\mathcal{E}} \widehat{\rho}\right)=\mathcal{Z}$, and the centre of $\mathcal{A} \rtimes^{\mathcal{E}} \widehat{\rho}$ is $\mathcal{Z}^{\rho}$.

Corollary 7.9. There is a dual action $\mu: \widehat{G} \rightarrow \widehat{\rho} \subset \mathbf{e n d} \mathcal{A}, \mathcal{A} \rtimes^{\mathcal{E}} \widehat{\rho}$ is isomorphic to the crossed product $\mathcal{A} \rtimes_{\mu} \widehat{G}$, and $G=\operatorname{aut}_{\mathcal{A}}\left(\mathcal{A} \rtimes^{\mathcal{E}} \widehat{\rho}, \sigma_{\mathcal{E}}\right)$.

Proof. First statement is consequence of Lemma [5.12 Second statement is consequence of Prop. 5.11 by recalling that $G=S\left(\widehat{\mathcal{Z}}^{\rho}, \mathcal{G}\right)$.

In the setting of previous theorem, let us consider the noncommutative pullback $\mathcal{M}$ defined by (5.15) (with $\mathcal{A} \rtimes^{\mathcal{E}} \widehat{\rho}$ playing the role of $\mathcal{A} \rtimes_{\mu} \widehat{G}$ ) carrying the natural $G$-action by bimodule maps. We can now state a duality result, consequence of Lemma 6.5

Corollary 7.10. Let $\rho$ be a special endomorphism of a unital $C^{*}$-algebra $\mathcal{A}$. Then for each vector bundle $\mathcal{E} \rightarrow \widehat{\mathcal{Z}}^{\rho}$ with rank $c_{0}(\rho)$ and first Chern class $c_{1}(\rho)$ there is a noncommutative pullback $\mathcal{M} \simeq \widehat{\mathcal{E}} \otimes_{\mathcal{Z}^{\rho}} \mathcal{Z}$ and a closed group $G \subseteq$ SUE acting on $\mathcal{M}$ by bimodule maps, with isomorphisms of strict tensor $C^{*}$ categories $\widehat{G} \simeq[\rho], \widehat{G}_{\mathcal{M}} \simeq \widehat{\rho}$. Furthermore the following diagram of morphisms of $C^{*}$-dynamical systems commutes:

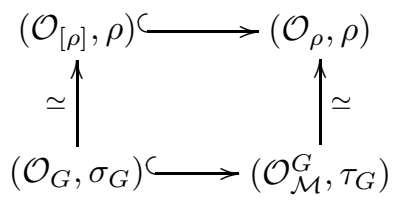

Remark 7.2. In the particular case in which $G$ is locally trivial, the crossed product in the sense of 6 by the semigroup generated by a single endomorphism can be regarded as a 'local coordinate' version of $\mathcal{A} \rtimes^{\mathcal{E}} \widehat{\rho}$.

Example 7.1. Let $\mathcal{E} \rightarrow X$ be a vector bundle, $G \subseteq \mathbf{S U E}$ a closed group. Then the canonical endomorphism $\sigma_{G}$ is special and $\mathcal{O}_{\mathcal{E}} \simeq \mathcal{O}_{G} \rtimes^{\mathcal{E}} \widehat{\sigma_{G}}$. We have $S(X, \mathcal{G}) \simeq \operatorname{aut}_{\mathcal{O}_{G}}\left(\mathcal{O}_{\mathcal{E}}\right)$, with isomorphisms of symmetric strict tensor $C^{*}$ categories $\widehat{\sigma}_{G} \simeq \widehat{G} \simeq(S(X, \mathcal{G}))$.

Example 7.2. Let $\mathcal{M} \simeq \widehat{\mathcal{E}} \otimes_{C(X)} C\left(X^{\prime}\right)$ be a noncommutative pullback, $G \subseteq$ SUE a closed group acting on $\mathcal{M}$ by $C\left(X^{\prime}\right)$-bimodule maps with $\mathcal{O}_{\mathcal{M}}^{G} \subseteq \mathcal{B}_{\mathcal{M}}$. The canonical endomorphism $\tau_{G}$ of $\mathcal{O}_{\mathcal{M}}^{G}$ is special (see Prop 6.4). By Prop 4.8 follows that $\mathcal{O}_{\mathcal{M}}=\mathcal{O}_{\mathcal{M}}^{G} \rtimes \mathcal{E} \widehat{\tau}_{G}$. Note that $\tau_{G}$ in general is not a $C\left(X^{\prime}\right)$-module map. We have $S(X, \mathcal{G}) \simeq \operatorname{aut}_{\mathcal{O}_{\mathcal{M}}^{G}}\left(\mathcal{O}_{\mathcal{M}}, \sigma_{\mathcal{E}}\right)$, with isomorphisms of strict tensor $C^{*}$-categories $\widehat{\tau}_{G} \simeq \widehat{G}_{\mathcal{M}},\left[\tau_{G}\right] \simeq \widehat{G}$. 


\section{Final Remarks (Work in Progress).}

Further step w.r.t. Thm 7.8 is the construction of crossed products by semigroups of special endomorphisms. A semigroup of special endomorphisms is said to be Permutation Symmetric, as by [19. Such a notion can be generalized according to Def 6.3 and Def 6.9 and the construction of corresponding crossed product is in progress.

Related to above exposed topic is duality for abstract tensor categories $\mathcal{T}$ with $C(X):=(\iota, \iota) \neq \mathbb{C}$, and with relaxed symmetry, conjugates as suggested by Def6.3. Def 6.9 Such a duality should give the abstract characterization for categories of (noncommutative pullbacks of) $G$-vector bundles. Note that we produced examples for which the following facts are verified:

- $\mathcal{T}$ admits several embeddings $i: \mathcal{T} \hookrightarrow \operatorname{vect}(X)$ into the category of vector bundles over $X$. For each embedding we obtain a characterization of $\mathcal{T}$ as the dual of a group $G_{i}$ spanning a spectral bundle $\mathcal{G}_{i} \rightarrow X$. Different embeddings give rise in general to not isomorphic groups and bundles (Ex 3.5);

- Fixed the embedding $i: \mathcal{T} \hookrightarrow \operatorname{vect}(X)$, not isomorphic groups having $\mathcal{T}$ as dual can be produced (Ex 3.3). Anyway such groups span the same spectral bundle.

An interesting problem is thus the classification of possible embeddings $\mathcal{T} \hookrightarrow$ $\operatorname{vect}(X)$.

Further point is the computation of $K$-theory for crossed products by dual actions $\mathcal{A} \rtimes_{\mu} \widehat{G}$, in terms of $K$-theory of $\mathcal{A}, \mathcal{O}_{G}, \mathcal{O}_{\mathcal{E}}$. Note that by functoriality the commutative diagram (5.1) induces a sequence at level of corresponding $K$ groups

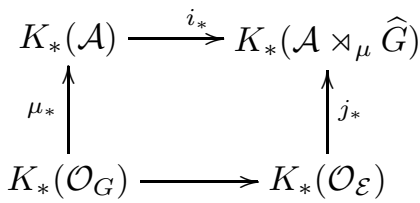

with $*=0,1$. At this purpose, $K$-theory of $\mathcal{O}_{\mathcal{E}}$ has been computed in the case in which $X$ is a $n$-sphere (41]). $K$-theory of $\mathcal{O}_{G}$ should depend only on the tensor $C^{*}$-category $\widehat{G}$, and could be related to $K$-theory of $\widehat{G}$ in the sense of 30. For $X=\{p t\}, K$-theory of $\mathcal{O}_{G}$ has been computed in 33.

Finally, the notion of braiding for $C^{*}$-algebra endomorphisms may be considered instead of symmetry, with condition $(\iota, \iota) \neq \mathbb{C}$, for applications to deformation quantization. In this setting, with notations of Cor [7.10 the generally not 
symmetric tensor category $\widehat{\rho} \simeq \widehat{G}_{\mathcal{M}}$ could play the role of a quantized category of $[\rho] \simeq \widehat{G}$, with $(\iota, \iota) \simeq C([0, \hbar])$ representing the space of Planck's constants, and $[\iota, \iota] \simeq \mathbb{C}$. In such a case $G$ is a Lie Group, and the tensor category $\widehat{G}_{\mathcal{M}}$ should correspond to a quantum group via a duality for braided $C^{*}$-categories of the type [13, 44. It is well known that braiding also appear in models in low dimensional quantum field theory (as in [2], 39]), together with condition of nontriviality of the centre (see discussions and references in [5], 4] $)$.

Acknowledgments. The author would like to thank S. Doplicher, for having proposed (and successively supported with improvements and encouragements) the initial idea of this work for his PhD Thesis, and G. Ruzzi, F. Ciolli, C. Pinzari, F. Lledó for precious help and stimulating discussions.

\section{References}

[1] B. Abadie, R. Exel: Hilbert $C^{*}$-bimodules over Commutative $C^{*}$-algebras and an isomorphism Condition for Quantum Heisemberg Manifolds, Reviews in Mathematical Physics 9(4) (1997), 411-423.

[2] F. Acerbi, G. Morchio, F. Strocchi: Infrared Singular Fields and nonregular Representations of Canonical Commutation Relation Algebras, J. Math. Phys. 34 (1993), 899-914.

[3] M.F. Atiyah: K-Theory, Benjamin, New York, 1967.

[4] H. Baumgärtel, A.L. Carey: Hilbert $C^{*}$-systems for Actions of the Circle Group, Rep. Math. Phys. 47(3) (2001), 349-361.

[5] H. Baumgärtel, F. Lledó: Superselection Structures for $C^{*}$-algebras with Nontrivial Center, Reviews in Mathematical Physics 9 (1997), 785-819.

[6] H. Baumgärtel, F. Lledó: An Application of the Doplicher-Roberts Duality for Compact Groups to Endomorphisms Categories of $C^{*}$-algebras with Nontrivial Center, in Mathematical Physics in Mathematics and Physics Quantum and Operator Algebraic Aspects, Fields Institute Communications 30 (2001), 1-10.

[7] B. Blackadar: K-Theory of Operator Algebras, 1995.

[8] E. Blanchard: Tensor Products of $C(X)$-algebras over $C(X)$, Astérisqque 232 (1995), 81-92.

[9] E. Blanchard: Déformations de $C^{*}$-algébres de Hopf, Bull. Soc. math. France 124 (1996), 141-215.

[10] P.J. Cohen: Factorization in Group Algebras, Duke Math. J. 26 (1959), 199-205. 
[11] J. Cuntz: The internal Structure of Simple $C^{*}$-Algebras, Pro. Sympos. Pure Math. 38 (1982), 85-115.

[12] J. Dixmier, A. Douady: Champs Continus d'Espaces Hilbertiens et de $C^{*}$ Algebres, Bull. Soc. math. France 91 (1963), 227-284.

[13] S. Doplicher, C. Pinzari, J.E. Roberts: An Algebraic Duality Theory for Multiplicative Unitaries, International Journal of Mathematics, 4(12) (2001), 415-459.

[14] S. Doplicher, C. Pinzari, R. Zuccante: The $C^{*}$-algebra of a Hilbert bimodule, Bollettino UMI, Serie VIII 1 B (1998), 263-282.

[15] S. Doplicher, J. E. Roberts: A Remark on Compact Automorphism Groups of $C^{*}$-Algebras, Journal of Functional Analysis 66 (1986), 67-72.

[16] S. Doplicher, J.E. Roberts: Duals of Compact Lie Groups Realized in the Cuntz Algebras and Their Actions on $C^{*}$-Algebras, Journal of Functional Analysis 74 (1987), 96-120.

[17] S. Doplicher, J.E. Roberts: Compact Group Actions on $C^{*}$-Algebras, Journal of Operator Theory 91 (1988), 227-284.

[18] S. Doplicher, J.E. Roberts: A New Duality Theory for Compact Groups, Inventiones Mathematicae 98 (1989), 157-218.

[19] S. Doplicher, J.E. Roberts: Endomorphisms of $C^{*}$-algebras, Cross Products and Duality for Compact Groups, Annals of Mathematics 130 (1989), 75119.

[20] S. Echterhoff, D.P. Williams: Crossed Products by certain $C_{0}(X)$-actions, J. Funct. Analysis 158 (1998), 113-151.

[21] S. Echterhoff, D.P. Williams: Locally Inner Actions on $C_{0}(X)$-algbras, Journal of Operator Theory 45 (2001), 131-160.

[22] R. Exel: A New Look at The Crossed-Product of a $C^{*}$-algebra by an Endomorphism, Preprint Universidade Federal de Santa Catarina, http://xxx.lanl.gov/abs/math.OA/0105195. (2000).

[23] I. Hirshberg: Endomorphisms of Stable Continuous Trace $C^{*}$-algebras, Preprint arXiv math.OA/0112293. (2001).

[24] M. Karoubi: K-Theory, Springer Verlag, Berlin - Heidelberg - New York, 1978.

[25] T. Kajiwara, C. Pinzari, Y. Watatani: Ideal Structure and Simplicity of the $C^{*}$-algebras generated by Hilbert bimodules, J. Funct. Analysis 159 (1998), 295-322. 
[26] T. Kajiwara, C. Pinzari, Y. Watatani: Hilbert $C^{*}$-bimodules and countably generated Cuntz-Krieger algebras, Journal of Operator Theory 45 (2001), $3-18$.

[27] G.G. Kasparov, Equivariant $K K$-Theory and the Novikov Conjecture, Invent. Math. 91 (1988), 147-201.

[28] R. Longo, J.E. Roberts: A Theory of Dimension, K-Theory 11 (1997), 103-159.

[29] A.S. Mishchenko, A.T. Fomenko: The Index of Elliptic Operators over C*-algebras, Math. USSR Izvestija, 1(15) (1980), 87-112.

[30] P.D. Mitchener: Symmetric $K$-theory Spectra of $C^{*}$-categories, $K$-theory 24(2) (2001), 157-201.

[31] M. Nilsen: $C^{*}$-Bundles and $C_{0}(X)$-algebras, Indiana Univ. Math. J. 45, (1996), 463-477.

[32] W. Paschke: The Crossed Product of a $C^{*}$-Algebra by an Endomorphism, Proceedings of the American Mathematical Society 80 (1980), 113-118.

[33] D. Pask, I. Raeburn: On the $K$-Theory of Cuntz-Krieger Algebras, Publ. RIMS Kyoto University 32 (1996), 415-443.

[34] M. Pimsner: A Class of $C^{*}$-algebras Generalizing both Cuntz-Krieger algebras and Cross Product by $\mathbb{Z}$, in: Free Probability Theory, Ed. D. Voiculescu, Fields Institute Communications 12 (1997), 189-212.

[35] C. Pinzari, Y. Watatani, K. Yonetani: KMS States, Entropy and the Variational Principle in Full $C^{*}$-dynamical Systems, Comm. Math. Phys. 213(2) (2001), 331-379.

[36] J. Phillips, I. Raeburn: Crossed Products by locally unitary automorphism groups and principal bundles, Journal of Operator Theory 11 (1984), 215241.

[37] J.E.Roberts, Unpublished Manuscript.

[38] P.J. Stacey: Crossed Products of $C^{*}$-Algebras by *-Endomorphisms, J. Austral. Math. Soc. Series A 54 (1993), 204-212.

[39] R. F. Streater, I. F. Wilde : Fermion States of a Boson Field, Nuclear Phys. B 24 (1970), 561-575.

[40] E. Vasselli: Continuous Fields of $C^{*}$-Algebras Arising from Extensions of Tensor $C^{*}$-Categories, to appear in Journal of Functional Analysis.

[41] E. Vasselli: Continuous Fields, $C^{*}$-Categories, Duality, and Crossed Products by Endomorphisms, PhD Thesis, Universitá di Roma Tor Vergata, 2002. 
[42] E. Vasselli: Some Remarks on Group Bundles and $C(X)$-dynamical Systems, Preprint arXiv math.OA/0301124 (2003), 14 pp.. Submitted.

[43] S. Waldmann: Morita Equivalence of Fedosov star products and deformed Hermitian vector bundles, Lett. Math. Phys., 60 (2002), 157-170.

[44] S.L. Woronowicz: Tannaka-Krein Duality for Compact Matrix Pseudogroups. Twisted $S U(N)$-groups, Invent. Math. 111 (1987). 